TRANSACTIONS OF THE

AMERICAN MATHEMATICAL SOCIETY

Volume 356, Number 10, Pages 4181-4236

S 0002-9947(04)03476-2

Article electronically published on April 16, 2004

\title{
VARIETIES OF TORI AND CARTAN SUBALGEBRAS OF RESTRICTED LIE ALGEBRAS
}

\author{
ROLF FARNSTEINER \\ Dedicated to Claus Michael Ringel on the occasion of his sixtieth birthday
}

\begin{abstract}
This paper investigates varieties of tori and Cartan subalgebras of a finite-dimensional restricted Lie algebra $(\mathfrak{g},[p])$, defined over an algebraically closed field $k$ of positive characteristic $p$. We begin by showing that schemes of tori may be used as a tool to retrieve results by A. Premet on regular Cartan subalgebras. Moreover, they give rise to principal fibre bundles, whose structure groups coincide with the Weyl groups in case $\mathfrak{g}=\operatorname{Lie}(\mathcal{G})$ is the Lie algebra of a smooth group $\mathcal{G}$. For solvable Lie algebras, varieties of tori are full affine spaces, while simple Lie algebras of classical or Cartan type cannot have varieties of this type. In the final sections the quasi-projective variety of Cartan subalgebras of minimal dimension $\operatorname{rk}(\mathfrak{g})$ is shown to be irreducible of dimension $\operatorname{dim}_{k} \mathfrak{g}-\mathrm{rk}(\mathfrak{g})$, with Premet's regular Cartan subalgebras belonging to the regular locus.
\end{abstract}

\section{INTRODUCTION}

In the structure theory of finite-dimensional complex Lie algebras the analysis of root systems associated to Cartan subalgebras plays a fundamental rôle. The utility of this approach partly rests on a conjugacy theorem, which renders root systems independent of the choice of a Cartan subalgebra. By contrast, the information obtained from the root system of a modular Lie algebra is well known to be highly sensitive to the choice of the defining Cartan subalgebra. The schemes of tori introduced in an earlier work [11] obviate this difficulty to some extent by affording the systematic investigation of algebraic families of Cartan subalgebras and root spaces. It is the main objective of this article to establish further properties of these smooth schemes, and to relate them to structural features of the underlying restricted Lie algebras.

Cartan subalgebras of modular Lie algebras have been studied extensively in the course of the last four decades. The main technique employed so far has been that of toral switching, which rests on generalizations of truncated exponentials that were first considered by Winter in [30] (see for instance [2] and [22, 23]). One aspect of our approach via schemes of tori is that it sheds new light on several results involving switching methods (see (3.5), (3.7) and (4.3)).

Much of our work employs standard facts from classical algebraic geometry, which we have collected in Section 1. However, the complexity of the defining

Received by the editors May 23, 2002 and, in revised form, July 19, 2003.

2000 Mathematics Subject Classification. Primary 17B50.

Supported by a Mercator Professorship of the D.F.G.

(C)2004 American Mathematical Society 
equations for the varieties of interest often necessitates the adoption of the functorial point of view, even though all schemes considered are reduced. For that reason schemes occasionally make an appearance at places where we could have equally well confined our attention to varieties.

Our paper is organized as follows. Having collected basic geometric results in a preliminary section, we turn in Section 2 to embeddings of tori and algebraic families of vector spaces. By way of illustration, we identify the scheme $\mathcal{T}_{\mathfrak{g}}$ of embeddings of a torus $\mathfrak{t}$ of a restricted Lie algebra $(\mathfrak{g},[p])$ in the "classical case", i.e., when $\mathfrak{g}=\operatorname{Lie}(\mathcal{G})$ is the Lie algebra of a smooth affine algebraic group $\mathcal{G}$.

Section 3 is mainly concerned with the generic containment of arbitrary tori of a restricted Lie algebra $\mathfrak{g}$ in tori of maximal dimension $\mu(\mathfrak{g})$. We re-establish those results from 22 that figure prominently in $\S 4$, where we study the geometric quotient $\hat{\mathcal{T}}_{\mathfrak{g}}$ of $\mathcal{T}_{\mathfrak{g}}$ consisting of the $d$-dimensional tori of $\mathfrak{g}$. If $d=\mu(\mathfrak{g})$ is maximal, then $\hat{\mathcal{T}}_{\mathfrak{g}}$ is an integral scheme, which is also the geometric quotient of a component $\mathcal{X}_{\mathfrak{t}} \subset \mathcal{T}_{\mathfrak{g}}$ by a finite group $S(\mathfrak{g}, \mathfrak{t})$. The structure group $S(\mathfrak{g}, \mathfrak{t})$ of the principal fibre bundle $\mathcal{X}_{\mathfrak{t}}(k) \longrightarrow \hat{\mathcal{T}}_{\mathfrak{g}}(k)$ coincides with the Weyl group in case $\mathfrak{g}$ is of classical type or the Lie algebra of a smooth group. Its isomorphism type turns out to be an interesting invariant of a restricted Lie algebra $(\mathfrak{g},[p])$.

The relationship between schemes of tori of an algebra and its factor algebras is investigated in Section 5. In general, the canonical projection $\mathfrak{g} \longrightarrow \mathfrak{g} / \mathfrak{n}$ induces a smooth morphism from certain components of the scheme $\mathcal{T}_{\mathfrak{g}}$ to the scheme $\mathcal{T}_{\mathfrak{g} / \mathfrak{n}}$ of the factor algebra $\mathfrak{g} / \mathfrak{n}$. This morphism is étale and an iterated vector bundle for toral and $p$-nilpotent ideals, respectively. An analogous result holds for the

morphism $\hat{\mathcal{T}}_{\mathfrak{g}} \longrightarrow \hat{\mathcal{T}}_{\mathfrak{g} / \mathfrak{n}}$. With this information in hand, we show in Section 6 that the scheme of maximal tori of a solvable Lie algebra of characteristic $\geq 3$ is a full affine space. By contrast, Weyl groups cannot occur as structure groups of principal fibre bundles $\mathcal{X}_{\mathfrak{t}}(k) \longrightarrow \mathbb{A}^{n}$, so that the schemes of tori of simple restricted Lie algebras of classical or Cartan type are not full affine spaces.

The second part of the paper is concerned with projective varieties. We employ algebraic families of tori and Cartan subalgebras to study the subvarieties of the Grassmannian of $\mathfrak{g}$ that are given by subalgebras of these types. By the classical conjugacy theorem these varieties are irreducible and smooth whenever $\mathfrak{g}=\operatorname{Lie}(\mathcal{G})$ is the Lie algebra of a smooth, connected algebraic $k$-group $\mathcal{G}$. In the general setting, the quasi-projective variety $\mathfrak{C} \mathfrak{a}(\mathfrak{g})$ of Cartan subalgebras of minimal dimension $\operatorname{rk}(\mathfrak{g})$ is shown to be irreducible and of dimension $\operatorname{dim} \mathfrak{C a r}(\mathfrak{g})=\operatorname{dim}_{k} \mathfrak{g}-\operatorname{rk}(\mathfrak{g})$. The elements of $\mathfrak{C a r}(\mathfrak{g})$ have generically isomorphic ascending central series, and their nilpotency class is generically constant. Moreover, the Cartan subalgebras of $\mathfrak{C a r}(\mathfrak{g})$ admitting a torus of maximal dimension belong to the regular locus of $\mathfrak{C a r}(\mathfrak{g})$. For solvable Lie algebras and simple restricted Lie algebras of classical or Cartan type the general structure theory then entails that the variety $\mathfrak{C} \mathfrak{a r}(\mathfrak{g})$ is smooth.

\section{Preliminaries}

In this section we collect a few basic results on morphisms of varieties that will be required throughout the paper. We will be working over a fixed algebraically closed field $k$. For general background we refer to [21]. Contrary to [21], however, a variety is not assumed to be irreducible.

Recall that a map $f: X \longrightarrow \mathbb{N}_{0}$ originating in a topological space $X$ is called upper semicontinuous if $f^{-1}\left(\left\{n \in \mathbb{N}_{0} ; n \geq d\right\}\right)$ is closed for every $d \in \mathbb{N}_{0}$. Given 
a morphism $\varphi: X \longrightarrow Y$ of varieties, we denote by $\varepsilon_{\varphi}(x):=\operatorname{dim}_{x} \varphi^{-1}(\varphi(x))$ the local dimension of $\varphi^{-1}(\varphi(x))$ at the point $x \in X$, i.e., the maximum dimension of those irreducible components of $\varphi^{-1}(\varphi(x))$ containing $x$. The following result is proved in [21, (I, $\S 8$, Cor.3)] for irreducible varieties.

1.1. Let $\varphi: X \longrightarrow Y$ be a morphism of varieties. Then the map

$$
X \longrightarrow \mathbb{N}_{0} \quad ; \quad x \mapsto \varepsilon_{\varphi}(x)
$$

is upper semicontinuous.

This readily yields:

Corollary 1.2. Let $\varphi: X \longrightarrow Y$ be a morphism of irreducible varieties such that $\operatorname{dim} X=\operatorname{dim} Y$. If there exists a point $x_{0} \in X$ such that $\varphi^{-1}\left(\varphi\left(x_{0}\right)\right)$ is finite, then $\varphi$ is dominant.

Proof. Let $Z:=\overline{\operatorname{im} \varphi}$. As $X$ is irreducible, our assumption in conjunction with (1.1) shows that $U:=\left\{x \in X ; \varepsilon_{\varphi}(x)=0\right\}$ is a dense, open subset of $X$. By [21, (I, $\S 8$, Cor.1)] there also exists a dense open subset $O \subset Z$ such that for every $y \in O$, every irreducible component of $\varphi^{-1}(y)$ has $\operatorname{dimension} \operatorname{dim} X-\operatorname{dim} Z$. As $X$ is irreducible, the open sets $U$ and $\varphi^{-1}(O)$ meet. Picking an element $x \in U \cap \varphi^{-1}(O)$ we obtain

$$
\operatorname{dim} X-\operatorname{dim} Z=\varepsilon_{\varphi}(x)=0 .
$$

Since $Y$ is irreducible of dimension $\operatorname{dim} X$, it follows that $Y=Z$, as desired.

Let $M_{k}$ and Ens be the categories of commutative $k$-algebras and sets, respectively. A local $k$-functor $\mathcal{X}: M_{k} \longrightarrow$ Ens that admits an open covering by affine algebraic schemes is called an algebraic scheme (cf. [16, (I, §1)]). For an arbitrary $k$-functor $\mathcal{X}: M_{k} \longrightarrow$ Ens, $x \in \mathcal{X}(R)$, and a homomorphism $\lambda: R \longrightarrow S$ of commutative $k$-algebras, we will occasionally suppress $\lambda$ and write $x_{S}=\mathcal{X}(\lambda)(x)$.

Recall that a morphism $\zeta: \mathcal{X} \longrightarrow \mathcal{Y}$ between two algebraic schemes is smooth if the following lifting property holds: For every commutative $k$-algebra $C$, and every ideal $I \triangleleft C$ with $I^{2}=(0)$, and every pair $(v, w) \in \mathcal{X}(C / I) \times \mathcal{Y}(C)$ with $\zeta_{C / I}(v)=$ $w_{C / I}$ there exists an element $u \in \mathcal{X}(C)$ such that $v=u_{C / I}$ and $\zeta_{C}(u)=w$. If there exists at most one such element $u \in \mathcal{X}(C)$, then $\zeta$ is unramified. A morphism that is unramified and smooth is called étale. In view of [6, (I, §4, 4.5, 5.11)]) it suffices to test the lifting property for finite-dimensional $k$-algebras $C$.

The interested reader may consult [21, (III, §5, and III, §10)] for more intuitive definitions of these notions. Due to the complexity of the defining equations the functorial definition for schemes is more useful in our context (see for instance [11. $(1.1)])$.

The coordinate ring of an affine variety $X$ will be denoted $k[X]$. Similarly, we will write $k[\mathcal{X}]$ for the coordinate ring of an affine scheme $\mathcal{X}$. If the schemes $\mathcal{X}$ and $\mathcal{Y}$ are affine, then the above notions correspond to those of the comorphism $\zeta^{*}: k[\mathcal{Y}] \longrightarrow k[\mathcal{X}]$ (cf. [20, p. 193]).

Given an affine variety $X$ and a finite group $G$ acting freely on $X$ via morphisms, we denote by $X / G$ the geometric quotient of $X$ by $G$ (cf. [16, (I.5.5(6))]). In view of [16, (I.5.5(6)] the affine scheme $X / G$ is reduced and thus again a variety. For future reference we record the following result on affine varieties with group action.

Lemma 1.3. Suppose that a finite group $G$ acts freely on the affine variety $X$. Then the canonical morphism $p: X \longrightarrow X / G$ is étale and faithfully flat. 
Proof. We put $Y:=X / G$. According to [6. (III, $\S 2,6.1$ )] the morphism $p$ is faithfully flat, and

$$
G \times X \longrightarrow X \times_{Y} X \quad ; \quad(g, x) \mapsto(g x, x)
$$

is an isomorphism. Since the projection $G \times X \longrightarrow X$ is obviously étale, the projection $\operatorname{pr}_{2}: X \times_{Y} X \longrightarrow X$ inherits this property. The assertion now follows by applying [18, (3.6.3.1)] to the universal diagram

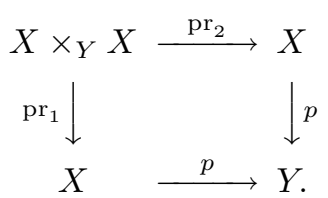

Lemma 1.4. Let $X \stackrel{f}{\longrightarrow} Y \stackrel{g}{\longrightarrow} Z$ be morphisms of varieties. If $g \circ f$ is étale, and $f$ is faithfully flat and étale, then $g$ is étale.

Proof. Without loss of generality, the varieties $X, Y$, and $Z$ may be assumed to be affine. Owing to [6. (I, $\S 4,4.7)]$ it suffices to show that $g$ is flat and unramified. The étale morphism $g \circ f$ is flat. In view of $f$ being faithfully flat, the flatness of $g$ follows.

Since $f$ is étale, the algebra $k[X]$ is smooth over $k[Y]$, and [20 (25.1)] yields a split exact sequence

$$
(0) \longrightarrow \Omega_{k[Y] / k[Z]} \otimes_{k[Y]} k[X] \longrightarrow \Omega_{k[X] / k[Z]} \longrightarrow \Omega_{k[X] / k[Y]} \longrightarrow(0)
$$

of Kähler differentials. As $g \circ f$ is unramified, the middle term vanishes. Accordingly, we have $\Omega_{k[Y] / k[Z]} \otimes_{k[Y]} k[X]=(0)$, which, in view of $f$ being faithfully flat, implies $\Omega_{k[Y] / k[Z]}=(0)$. Hence the morphism $g$ is unramified.

The following result is adapted from [26, Chapter I, $\S 6$, Thm.7].

Proposition 1.5. Let $\varphi: X \longrightarrow Y$ be a surjective morphism of varieties such that

(a) $Y$ is irreducible, and

(b) the image $\varphi(Z)$ of each irreducible component $Z$ of $X$ is closed in $Y$, and

(c) there exists $r \in \mathbb{N}_{0}$ such that $\varphi^{-1}(y)$ is irreducible of dimension $r$ for every $y \in Y$.

Then $X$ is irreducible.

Proof. We decompose $X$ into its irreducible components, $X=\bigcup_{i=1}^{n} X_{i}$, and first verify

$$
\varphi^{-1}\left(\varphi\left(X_{i}\right)\right)=X_{i} \text { for every } i \in\{1, \ldots, n\} .
$$

Consider the nonempty, open subset $O_{i}:=X_{i} \backslash \bigcup_{j \neq i} X_{j}$ of $X_{i}$. Let $x \in O_{i}$. By property (c) there is $\ell \in\{1, \ldots, n\}$ such that $\varphi^{-1}(\varphi(x)) \subset X_{\ell}$. As $x \in \varphi^{-1}(\varphi(x)) \cap$ $O_{i}$, we conclude that $\ell=i$.

General theory, applied to the morphism $\left.\varphi\right|_{X_{i}}: X_{i} \longrightarrow \overline{\varphi\left(X_{i}\right)}$, shows that there is a dense open subset $U \subset \varphi\left(X_{i}\right)$ of $\overline{\varphi\left(X_{i}\right)}$ such that $\operatorname{dim} \varphi^{-1}(y) \cap X_{i}=\operatorname{dim} X_{i}-$ $\operatorname{dim} \overline{\varphi\left(X_{i}\right)}$ for $y \in U$, and $\operatorname{dim} \varphi^{-1}(y) \cap X_{i} \geq \operatorname{dim} X_{i}-\operatorname{dim} \overline{\varphi\left(X_{i}\right)}$ for $y \in \varphi\left(X_{i}\right)$ (cf. [21, (I, $\S 8$, Cor., Cor.1)]). Since $X_{i}$ is irreducible, we have $O_{i} \cap\left(\left.\varphi\right|_{X_{i}}\right)^{-1}(U) \neq \emptyset$, 
whence $\operatorname{dim} X_{i}-\operatorname{dim} \overline{\varphi\left(X_{i}\right)}=r$. Consequently,

$$
\operatorname{dim} \varphi^{-1}(y) \cap X_{i} \geq r=\operatorname{dim} \varphi^{-1}(y) \quad \forall y \in \varphi\left(X_{i}\right),
$$

so that (c) yields $\varphi^{-1}(y)=\varphi^{-1}(y) \cap X_{i} \subset X_{i}$ for every such $y$. As a result, (*) holds.

Since $\varphi$ is surjective and $Y$ is irreducible, condition (b) implies that $\varphi\left(X_{i_{0}}\right)=Y$ for some $i_{0} \in\{1, \ldots, n\}$. Consequently, $(*)$ shows that

$$
X=\varphi^{-1}(Y)=\varphi^{-1}\left(\varphi\left(X_{i_{0}}\right)\right)=X_{i_{0}}
$$

is irreducible.

Remark. The technical condition (b) is essential to the validity of (1.5). The variety $X:=\left\{(a, b) \in k^{2} ; a b=1\right\} \cup\{(0,0)\}$ is reducible with two irreducible components. If $\varphi$ is the restriction to $X$ of the projection onto the first coordinate, then $\varphi$ is surjective with each fibre being a singleton. Since $\varphi\left(\left\{(a, b) \in k^{2} ; a b=1\right\}\right)=k \backslash\{0\}$, condition (b) of (1.5) does not hold.

We conclude this preparatory section by applying the above observations to algebraic families of vector spaces. To that end, we let $A$ and $V$ be a finitely generated integral domain over $k$, and a finite-dimensional $k$-vector space, respectively. We consider the affine algebraic scheme $\mathcal{X}:=\operatorname{Spec}_{k}(A)$, and let $P \subset V \otimes_{k} A$ be an $A$-direct summand of the free $A$-module $V \otimes_{k} A$. Given a subvariety $Y \subset V$ we put

$$
Y_{P, \mathcal{X}}:=\left\{(x, y) \in \mathcal{X}(k) \times Y ; \exists p \in P \text { such that }\left(\operatorname{id}_{V} \otimes x\right)(p)=y\right\} .
$$

Since $Y_{P, \mathcal{X}}=V_{P, \mathcal{X}} \cap(\mathcal{X}(k) \times Y)$, and $V_{P, \mathcal{X}} \subset \mathcal{X}(k) \times V$ is closed, it follows that $Y_{P, \mathcal{X}}$ is a subvariety of $\mathcal{X}(k) \times Y$ (cf. [11, (2.5)]). The canonical projections induce morphisms $\pi_{\mathcal{X}}: Y_{P, \mathcal{X}} \longrightarrow \mathcal{X}(k)$ and $\pi_{Y}: Y_{P, \mathcal{X}} \longrightarrow Y$.

For any $x \in \mathcal{X}(k)$ we set $P(x):=\left(\operatorname{id}_{V} \otimes x\right)(P) \subset V$. Since $P$ is a finitely generated projective module over an integral domain, it has constant $\operatorname{rank} \operatorname{rk}(P)$ (cf. [20, p. 166]). This readily implies $\operatorname{dim}_{k} P(x)=\operatorname{rk}(P)$ for every $x \in \mathcal{X}(k)$.

Proposition 1.6. Let $Y \subset V$ be a closed, conical subvariety.

(1) The function $\mathcal{X}(k) \longrightarrow \mathbb{N}_{0} ; x \mapsto \operatorname{dim} P(x) \cap Y$ is upper semicontinuous.

(2) If there exists $\ell \in \mathbb{N}_{0}$ such that $Y \cap P(x)$ is irreducible and of dimension $\ell$ for every $x \in \mathcal{X}(k)$, then $Y_{P, \mathcal{X}}$ is irreducible and of dimension $\operatorname{dim} Y_{P, \mathcal{X}}=\operatorname{dim} \mathcal{X}(k)+\ell$.

(3) The variety $V_{P, \mathcal{X}}$ is irreducible with $\operatorname{dim} V_{P, \mathcal{X}}=\operatorname{dim} \mathcal{X}(k)+\operatorname{rk}(P)$.

Proof. (1) As $Y$ is closed and conical, we have $0 \in Y$. Thus, the projection $\pi_{\mathcal{X}}$ is surjective and the morphism

$$
\iota_{0}: \mathcal{X}(k) \longrightarrow Y_{P, \mathcal{X}} \quad ; \quad x \mapsto(x, 0)
$$

is well defined. It follows from (1.1) that the map

$$
\mathcal{X}(k) \longrightarrow \mathbb{N}_{0} \quad ; \quad x \mapsto \operatorname{dim}_{(x, 0)} \pi_{\mathcal{X}}^{-1}\left(\pi_{\mathcal{X}}(x, 0)\right)
$$

is upper semicontinuous. Since $\pi_{Y}$ induces an isomorphism $\pi_{\mathcal{X}}^{-1}(x) \cong P(x) \cap Y$ for every element $x \in \mathcal{X}(k)$, we have $\operatorname{dim}_{(x, 0)} \pi_{\mathcal{X}}^{-1}\left(\pi_{\mathcal{X}}(x, 0)\right)=\operatorname{dim}_{0} P(x) \cap Y$. As $P(x) \cap Y$ is conical, each of its irreducible components contains 0 , whence $\operatorname{dim}_{0} P(x) \cap Y=\operatorname{dim} P(x) \cap Y$. Consequently, the map $x \mapsto \operatorname{dim} P(x) \cap Y$ is upper semicontinuous.

(2) The connected algebraic group $k^{\times}$operates on $Y_{P, \mathcal{X}}$ via

$$
\alpha \cdot(x, y):=(x, \alpha y) \text {. }
$$


Hence every irreducible component $Z \subset Y_{P, \mathcal{X}}$ is $k^{\times}$-invariant, so that $(x, 0) \in Z$ whenever $(x, v) \in Z$. Consequently, $\pi_{\mathcal{X}}(Z)=\iota_{0}^{-1}(Z)$ is closed. Since $\mathcal{X}(k)$ is irreducible, assertion (2) is now a direct consequence of (1.5) and [21, (I, §8, Cor.1)].

(3) This follows directly from (2) and the observation preceding (1.6).

\section{The SCHEME OF EMBEDDINGS OF A TORUS}

Henceforth the algebraically closed field $k$ is assumed to have characteristic $p>0$. A restricted Lie algebra is a pair $(\mathfrak{g},[p])$ consisting of a Lie algebra $\mathfrak{g}$ and an operator

$$
\mathfrak{g} \longrightarrow \mathfrak{g} ; x \mapsto x^{[p]}
$$

that satisfies the formal properties of a $p$-power map. We refer the reader to [29]. Chapter II] for basic facts on restricted Lie algebras. Recall that

$$
\mathcal{V}_{\mathfrak{g}}:=\left\{x \in \mathfrak{g} ; x^{[p]}=0\right\}
$$

is the nullcone of $\mathfrak{g}$, and that $\mathfrak{g}$ is a torus if $\mathcal{V}_{\mathfrak{g}}=\{0\}$. Tori are necessarily abelian Lie algebras (cf. [29, (II.3.10)]). We let Tor( $\mathfrak{g})$ be the set of maximal tori of $\mathfrak{g}$. The maximal dimension of an element of $\operatorname{Tor}(\mathfrak{g})$ will be denoted $\mu(\mathfrak{g})$.

Given a finite-dimensional restricted Lie algebra $(\mathfrak{g},[p])$ over $k$ and a commutative $k$-algebra $R$, the $R$-module $\mathfrak{g} \otimes_{k} R$ obtains the structure of a restricted $R$-Lie algebra via

$$
[x \otimes r, y \otimes s]:=[x, y] \otimes r s, \quad(x \otimes r)^{[p]}:=x^{[p]} \otimes r^{p} \quad \forall x, y \in \mathfrak{g}, r, s \in R .
$$

Accordingly, $\mathfrak{g}$ can be considered a $k$-functor $M_{k} \longrightarrow$ Ens.

Let $\mathfrak{t} \subset \mathfrak{g}$ be a torus, $R$ a commutative $k$-algebra. We define $\mathcal{T}_{\mathfrak{g}}(R)$ to be the set of homomorphisms $\mathfrak{t} \otimes_{k} R \longrightarrow \mathfrak{g} \otimes_{k} R$ of restricted $R$-Lie algebras that are split injective when considered $R$-linear maps. The resulting $k$-functor $\mathcal{T}_{\mathfrak{g}}: M_{k} \longrightarrow$ Ens is the scheme of embeddings from $\mathfrak{t}$ to $\mathfrak{g}$. According to [1], (1.4)] the algebraic affine scheme $\mathcal{T}_{\mathfrak{g}}$ is smooth. Consequently, the connected components of $\mathcal{T}_{\mathfrak{g}}$ are irreducible, and there exists precisely one irreducible component $\mathcal{X}_{\mathfrak{t}} \subset \mathcal{T}_{\mathfrak{g}}$ such that the given embedding $\mathfrak{t} \hookrightarrow \mathfrak{g}$ belongs to $\mathcal{X}_{\mathfrak{t}}(k)$.

An element of $\mathcal{T}_{\mathfrak{g}}(R)$ gives rise to an injective homomorphism $\varphi: \mathfrak{t} \longrightarrow \mathfrak{g} \otimes_{k} R$ of restricted $k$-Lie algebras such that $R \varphi(\mathfrak{t})$ is a direct summand of the $R$-module $\mathfrak{g} \otimes_{k} R$. When convenient, the elements of $\mathcal{T}_{\mathfrak{g}}(R)$ will be viewed in this fashion.

Let $\mathcal{X} \subset \mathcal{T}_{\mathfrak{g}}$ be an irreducible component. Since $\mathcal{X}$ is reduced, it is an integral affine algebraic scheme, and we write $\mathcal{X} \cong \operatorname{Spec}_{k}(A)$, where $A$ is a finitely generated integral domain over $k$. By Yoneda's Lemma the isomorphism is completely determined by the image $j \in \mathcal{X}(A)$ of $\operatorname{id}_{A} \in \operatorname{Spec}_{k}(A)(A)$. The corresponding injective map $j: \mathfrak{t} \hookrightarrow \mathfrak{g} \otimes_{k} A$ of restricted $k$-Lie algebras is referred to as the universal embedding of $\mathfrak{t}$ (relative to $\mathcal{X}$ ). An element $\varphi_{x}: \mathfrak{t} \longrightarrow \mathfrak{g} \otimes_{k} R$ corresponds to $x \in \operatorname{Spec}_{k}(A)(R)$ if and only if

$$
\varphi_{x}=\left(\mathrm{id}_{\mathfrak{g}} \otimes x\right) \circ j .
$$

In the sequel we will often identify elements of $\operatorname{Spec}_{k}(A)(R)$ and $\mathcal{X}(R)$ in this fashion.

Consider a restricted $\mathfrak{g}$-module $M$. By definition, each element $x^{[p]}$ operates on $M$ via the $p$-th iterate of $x$, i.e., $x^{[p]} m=x^{p} m$ for every $x \in \mathfrak{g}$ and $m \in M$. As $\tilde{M}:=M \otimes_{k} A$ is a restricted module for the restricted Lie algebra $\tilde{\mathfrak{g}}:=\mathfrak{g} \otimes_{k} A$, the universal embedding $j: \mathfrak{t} \hookrightarrow \tilde{\mathfrak{g}}$ endows the $A$-module $\tilde{M}$ with the structure of a restricted $\mathfrak{t}$-module on which $\mathfrak{t}$ operates via $A$-linear transformations. To a 
linear form $\gamma \in \mathfrak{t}^{*}$ satisfying $\gamma(t)^{p}=\gamma\left(t^{[p]}\right)$ for every $t \in \mathfrak{t}$ we associate the $A$ module $\tilde{M}_{\gamma}:=\{v \in \tilde{M} ; j(t) v=\gamma(t) v \forall t \in \mathfrak{t}\}$. There results a weight space decomposition

$$
\tilde{M}:=\bigoplus_{\gamma \in \Gamma} \tilde{M}_{\gamma},
$$

where $\Gamma \subset \mathfrak{t}^{*}$ is finite and $\tilde{M}_{\gamma} \neq(0)$ for every $\gamma \in \Gamma$. For $x \in \operatorname{Spec}_{k}(A)(k)$ the subspace $\tilde{M}_{\gamma}(x) \subset M$ is the weight space $M_{\gamma \circ \varphi_{x}^{-1}}$ relative to the torus $\varphi_{x}(\mathfrak{t}) \subset \mathfrak{g}$. In particular, if $\mathcal{X}=\mathcal{X}_{\mathbf{t}}$ and the originally given inclusion $\mathfrak{t} \hookrightarrow \mathfrak{g}$ corresponds to $x_{0} \in \operatorname{Spec}_{k}(A)(k)$, then

$$
M=\bigoplus_{\gamma \in \Gamma} \tilde{M}_{\gamma}\left(x_{0}\right)
$$

is the weight space decomposition of $M$ relative to $\mathfrak{t}$.

By letting $\mathfrak{g}$ act on itself via the adjoint representation, we obtain a decomposition

$$
\tilde{\mathfrak{g}}=\tilde{\mathfrak{g}}_{0} \oplus \bigoplus_{\alpha \in \Phi} \tilde{\mathfrak{g}}_{\alpha},
$$

where $\Phi \subset \mathfrak{t}^{*} \backslash\{0\}$. For $\mathcal{X}=\mathcal{X}_{\mathfrak{t}}$ this decomposition specializes to the root space decomposition of $\mathfrak{g}$ relative to $\mathfrak{t}$.

Let $V \subset \mathfrak{g}$ be a subset. The $p$-subalgebra $C_{\mathfrak{g}}(V):=\{x \in \mathfrak{g} ;[x, v]=0 \quad \forall v \in V\}$ is called the centralizer of $V$ in $\mathfrak{g}$. For example, we have $\tilde{\mathfrak{g}}_{0}(x)=C_{\mathfrak{g}}\left(\varphi_{x}(\mathfrak{t})\right)$ for every element $x \in \operatorname{Spec}_{k}(A)(k)$. We write $C(\mathfrak{g}):=C_{\mathfrak{g}}(\mathfrak{g})$ for the center of $\mathfrak{g}$.

We begin by describing the scheme $\mathcal{T}_{\mathfrak{g}}$ for Lie algebras of smooth (reduced) groups, and denote for every commutative $k$-algebra $R$ the category of all commutative $R$-algebras by $M_{R}$. Let $\mathcal{G}$ be an affine algebraic $k$-group with Lie algebra $\mathfrak{g}:=\operatorname{Lie}(\mathcal{G})$, and consider its adjoint representation $\mathrm{Ad}: \mathcal{G} \longrightarrow \mathcal{A U} \mathcal{T}(\operatorname{Lie}(\mathcal{G}))$. If $\mathfrak{t} \subset \mathfrak{g}$ is a torus, then $\operatorname{Cent}_{\mathcal{G}}(\mathfrak{t})$ is the centralizer of the subfunctor $\mathfrak{t} \subset \mathfrak{g}$. By definition (see [16, (I.2.6)]) we have

$$
\operatorname{Cent}_{\mathcal{G}}(\mathfrak{t})(R)=\left\{g \in \mathcal{G}(R) ; \operatorname{Ad}\left(g_{R^{\prime}}\right)(t)=t \quad \forall t \in \mathfrak{t} \otimes_{k} R^{\prime} \text { and all } R^{\prime} \in M_{R}\right\}
$$

for every $R \in M_{k}$. If $\mathcal{G}$ is smooth, then [16, (I.2.6(12))] yields $\operatorname{Cent}_{\mathcal{G}(k)}(\mathfrak{t})=$ $\operatorname{Cent}_{\mathcal{G}}(\mathfrak{t})(k)$. The group scheme $\mathcal{G}$ operates on the affine scheme $\mathcal{T}_{\mathfrak{g}}$ via

$$
g \cdot \varphi:=\operatorname{Ad}(g) \circ \varphi \quad \forall g \in \mathcal{G}(R), \varphi \in \mathcal{T}_{\mathfrak{g}}(R) \text { and all } R \in M_{k}
$$

Theorem 2.1. Let $\mathfrak{g}=\operatorname{Lie}(\mathcal{G})$ be the Lie algebra of a smooth, connected, affine algebraic $k$-group $\mathcal{G}, \mathfrak{t} \subset \mathfrak{g}$ a torus. Then the following statements hold:

(1) The connected components of $\mathcal{T}_{\mathfrak{g}}$ are the $\mathcal{G}$-orbits in $\mathcal{T}_{\mathfrak{g}}(k)$.

(2) The action of $\mathcal{G}$ on $\mathcal{T}_{\mathfrak{g}}$ induces an isomorphism $\mathcal{G} /$ Cent $_{\mathcal{G}}(\mathfrak{t}) \cong \mathcal{X}_{\mathfrak{t}}$ of schemes.

Proof. Let $\mathcal{X} \subset \mathcal{T}_{\mathfrak{g}}$ be a connected component, $\varphi \in \mathcal{X}(k)$ a rational point. According to [16, (I.2.6(9))] the stabilizer $\operatorname{Stab}_{\mathcal{G}}(\mathcal{X})$ of $\mathcal{X}$ in $\mathcal{G}$ is a closed subgroup of $\mathcal{G}$. Since the orbit $\mathcal{G}(k) \cdot \varphi$ is connected, we have $g \cdot \varphi \in \mathcal{X}(k)$ for every $g \in \mathcal{G}(k)$. Thus, [16, (I.2.6(12))] implies

$$
\mathcal{G}(k)=\operatorname{Stab}_{\mathcal{G}(k)}(\mathcal{X}(k))=\operatorname{Stab}_{\mathcal{G}}(\mathcal{X})(k) .
$$

As $\mathcal{G}$ is reduced, $\mathcal{G}(k)$ is dense in $\mathcal{G}$, so that $\mathcal{G}=\operatorname{Stab}_{\mathcal{G}}(\mathcal{X})$. Consequently, the group $\mathcal{G}$ operates on $\mathcal{X}$.

Note that

$$
\operatorname{Stab}_{\mathcal{G}(k)}(\varphi)=\{g \in \mathcal{G}(k) ; \operatorname{Ad}(g)(\varphi(t))=\varphi(t) \quad \forall t \in \mathfrak{t}\} .
$$


Thus, $\operatorname{Lie}\left(\operatorname{Stab}_{\mathcal{G}(k)}(\varphi)\right) \subset C_{\mathfrak{g}}(\varphi(\mathfrak{t}))$, and [11, (1.6)] yields

$$
\operatorname{dim} \overline{\mathcal{G}(k) \cdot \varphi}=\operatorname{dim} \mathcal{G}(k)-\operatorname{dim} \operatorname{Stab}_{\mathcal{G}(k)}(\varphi) \geq \operatorname{dim}_{k} \mathfrak{g}-\operatorname{dim}_{k} C_{\mathfrak{g}}(\varphi(\mathfrak{t}))=\operatorname{dim} \mathcal{X} .
$$

Hence every orbit of $\mathcal{X}(k)$ lies dense in $\mathcal{X}(k)$. Consequently, every orbit is closed, and $\mathcal{X}(k)=\mathcal{G}(k) \cdot \varphi$.

By the above, the morphism

$$
\gamma: \mathcal{G} \longrightarrow \mathcal{X} \quad ; \quad g \mapsto \operatorname{Ad}(g) \circ \varphi
$$

given by

$$
\gamma_{R}(g)=\operatorname{Ad}(g) \circ \varphi_{R} \quad \forall R \in M_{k}, g \in \mathcal{G}(R)
$$

induces a bijective $\mathcal{G}(k)$-equivariant map $\omega: \mathcal{G}(k) / \operatorname{Stab}_{\mathcal{G}(k)}(\varphi) \longrightarrow \mathcal{X}(k)$ of homogeneous spaces. Since

$$
\begin{aligned}
\operatorname{dim}_{k} C_{\mathfrak{g}}(\varphi(\mathfrak{t})) & =\operatorname{dim}_{k} \mathfrak{g}-\operatorname{dim} \mathcal{X}(k)=\operatorname{dim}_{k} \mathfrak{g}-\operatorname{dim}(\mathcal{G}(k) \cdot \varphi)=\operatorname{dim} \operatorname{Stab}_{\mathcal{G}(k)}(\varphi) \\
& =\operatorname{dim}_{\operatorname{Cent}_{\mathcal{G}(k)}(\varphi(\mathfrak{t})),}
\end{aligned}
$$

we obtain for the tangent space $T_{\overline{1}}\left(\mathcal{G}(k) / \operatorname{Cent}_{\mathcal{G}(k)}(\varphi(\mathfrak{t}))\right)$ at the image $\overline{1}$ of the identity element $1 \in \mathcal{G}(k)$

$$
\begin{aligned}
& \operatorname{dim}_{k} T_{\overline{1}}\left(\mathcal{G}(k) / \operatorname{Cent}_{\mathcal{G}(k)}(\varphi(\mathfrak{t}))\right)=\operatorname{dim}_{\mathcal{G}}(k) / \operatorname{Cent}_{\mathcal{G}(k)}(\varphi(\mathfrak{t})) \\
& =\operatorname{dim} \mathcal{G}(k)-\operatorname{dim}_{\operatorname{Cent}} \operatorname{G(k)}_{(\varphi(\mathfrak{t}))} \\
& =\operatorname{dim}_{k} T_{\varphi}(\mathcal{X}) \text {. }
\end{aligned}
$$

Let $\operatorname{Der}_{p}(\varphi(\mathfrak{t}), \mathfrak{g})$ be the space of derivations $\zeta: \varphi(\mathfrak{t}) \longrightarrow \mathfrak{g}$ satisfying $\zeta\left(t^{[p]}\right)=$ $(\operatorname{ad} t)^{p-1}(\zeta(t))$ for every $t \in \varphi(\mathfrak{t})$. Thanks to [11, (1.4),(1.5)] this space coincides with the tangent space $T_{\varphi}(\mathcal{X})$. The differential of $\gamma$ at the identity is given by

$$
(d \gamma)_{1}: \mathfrak{g} \longrightarrow \operatorname{Der}_{p}(\varphi(\mathfrak{t}), \mathfrak{g}) ;\left.x \mapsto \operatorname{ad} x\right|_{\varphi(\mathfrak{t})} .
$$

Since $\varphi(\mathfrak{t})$ is a torus (cf. [29] (II.4.5)]), an application of [13, (2.1)] shows that this map is surjective. Consequently, the tangent map $(d \omega)_{\overline{1}}: T_{\overline{1}}\left(\mathcal{G}(k) / \operatorname{Cent}_{\mathcal{G}(k)}(\varphi(\mathfrak{t}))\right)$ $\longrightarrow \operatorname{Der}_{p}(\varphi(\mathfrak{t}), \mathfrak{g})$ also enjoys this property. As both spaces have the same dimension, $(d \omega)_{\overline{1}}$ is bijective, and [28, (4.3.3)] implies that $\omega$ is in fact an isomorphism.

Now let $\bar{\gamma}: \mathcal{G} / \operatorname{Stab}_{\mathcal{G}}(\varphi) \longrightarrow \mathcal{X}$ be the morphism induced by $\gamma$. In view of 6. (III, $\S 3,5.4)$ ] the $k$-functor $\mathcal{G} / \operatorname{Stab}_{\mathcal{G}}(\varphi)$ is a scheme, whose rational points are given by $\mathcal{G}(k) / \operatorname{Stab}_{\mathcal{G}(k)}(\varphi)$ (cf. [ㅁ, (III, $\left.\left.\S 1,1.15\right)\right]$ ). By the above, the map $\bar{\gamma}_{k}$ is an isomorphism of varieties. Since the schemes $\mathcal{G} / \operatorname{Stab}_{\mathcal{G}}(\varphi)$ and $\mathcal{X}$ are reduced, it follows from [6, $(\mathrm{I}, \S 3,6.8,6.9)]$ that $\bar{\gamma}$ is an isomorphism. This verifies (1) and (2) follows by considering $\mathcal{X}_{\mathfrak{t}}$ along with the given embedding $\mathfrak{t} \hookrightarrow \mathfrak{g}$.

Remark. (1) As a consequence we recover classical facts on centralizers of semisimple elements (cf. [4, (III.9.1)]). Let $s \in \mathfrak{g}$ be semisimple, and consider the torus $\mathfrak{t}:=$ $\sum_{i \geq 0} k s^{[p]^{i}}$. Owing to (2.1) and [11, (1.6)] we have $C_{\mathfrak{g}}(s)=C_{\mathfrak{g}}(\mathfrak{t})=\operatorname{Lie}\left(\operatorname{Cent}_{\mathcal{G}(k)}(\mathfrak{t})\right)$ $=\operatorname{Lie}\left(\operatorname{Cent}_{\mathcal{G}(k)}(s)\right)$. By the same token, the variety $\mathcal{G}(k) / \operatorname{Cent}_{\mathcal{G}(k)}(s)$ is affine.

(2) Theorem 2.1 does not hold for an arbitrary finite-dimensional restricted Lie algebra $(\mathfrak{g},[p])$, that is, the group $\mathcal{G}$ may not be replaced by the connected component of the automorphism group of $\mathfrak{g}$. Consider, for instance, the Witt-algebra $W(1):=\bigoplus_{i=-1}^{p-2} k e_{i}$ with bracket and $p$-map

$$
\left[e_{i}, e_{j}\right]=(j-i) e_{i+j} ; \quad e_{i}^{[p]}=\delta_{i 0} e_{0} .
$$

Owing to [7] the space $\mathfrak{t}:=k e_{0}$ is a maximal torus of $W(1)$, whose conjugates under $\operatorname{Aut}_{p}(W(1))^{\circ}$ are contained in the $p$-subalgebra $W(1)_{(0)}:=\sum_{i \geq 0} k e_{i}$. Hence, if 
$\mathcal{X}_{\mathfrak{t}}(k)$ is the orbit of the standard embedding, then $\mathcal{X}_{\mathfrak{t}}(k)$ is a connected component of $\mathcal{T}_{W(1)_{(0)}}(k)$, so that [11, (1.6)] implies $\operatorname{dim} \mathcal{X}_{\mathbf{t}}(k)=p-2$. By applying the same result to $\mathcal{X}_{\mathfrak{t}}(k) \subset \mathcal{T}_{W(1)}(k)$, we have $\operatorname{dim} \mathcal{X}_{\mathfrak{t}}(k)=p-1$, a contradiction.

We return to our general set-up, and let $(\mathfrak{g},[p])$ be an arbitrary finite-dimensional restricted Lie algebra, $\mathfrak{t} \subset \mathfrak{g}$ a torus.

Proposition 2.2. Let $\mathcal{X} \subset \mathcal{T}_{\mathfrak{g}}$ be an irreducible component, $\varphi$ an element of $\mathcal{X}(k)$. If $P \subset \tilde{\mathfrak{g}}$ is a $k[\mathcal{X}]$-direct summand, then the variety $\mathfrak{g}_{P, \mathcal{X}}$ is irreducible and of dimension $\operatorname{dim} \mathfrak{g}_{P, \mathcal{X}}=\operatorname{dim}_{k} \mathfrak{g} / C_{\mathfrak{g}}(\varphi(\mathfrak{t}))+\operatorname{rk}(P)$. In particular, we have $\operatorname{dim} \mathfrak{g}_{\tilde{g}_{0}, \mathcal{X}}=$ $\operatorname{dim}_{k} \mathfrak{g}$.

Proof. The first statement follows from a consecutive application of (1.6(3)) and [11, (1.6)]. Since $\operatorname{rk}\left(\tilde{\mathfrak{g}}_{0}\right)=\operatorname{dim}_{k} C_{\mathfrak{g}}(\varphi(\mathfrak{t}))$ we have $\operatorname{dim} \mathfrak{g}_{\tilde{g}_{0}, \mathcal{X}}=\operatorname{dim}_{k} \mathfrak{g}$.

If $\mathfrak{h} \subset \mathfrak{g}$ is a $p$-subalgebra, then $\mathcal{T}_{\mathfrak{h}}(k)$ will be identified with the closed subvariety of $\mathcal{T}_{\mathfrak{g}}(k)$ consisting of those injections whose images are contained in $\mathfrak{h}$.

Proposition 2.3. Let $\mathcal{X} \subset \mathcal{T}_{\mathfrak{g}}$ be an irreducible component, $v \in \mathfrak{g}$. If $P \subset \tilde{\mathfrak{g}}_{0}$ is a $k[\mathcal{X}]$-direct summand of $\tilde{\mathfrak{g}}$, then the morphism $\pi_{\mathcal{X}}: \mathfrak{g}_{P, \mathcal{X}} \longrightarrow \mathcal{X}(k)$ induces an embedding $\pi_{\mathfrak{g}}^{-1}(v) \hookrightarrow \mathcal{T}_{C_{\mathfrak{g}}(v)}(k)$.

Proof. We put $A:=k[\mathcal{X}]$. Note that $(x, b) \in \pi_{\mathfrak{g}}^{-1}(v)$ if and only if $b=v$ and $v \in$ $P(x)$. Since $P$ is contained in $\tilde{\mathfrak{g}}_{0}$, we have $v \in \tilde{\mathfrak{g}}_{0}(x)=C_{\mathfrak{g}}\left(\varphi_{x}(\mathfrak{t})\right)$, so that $\varphi_{x}(\mathfrak{t}) \subset$ $C_{\mathfrak{g}}(v)$. Accordingly, the projection $\pi_{\mathcal{X}}: \mathfrak{g}_{P, \mathcal{X}} \longrightarrow \mathcal{X}(k)$ induces an embedding $\pi_{\mathfrak{g}}^{-1}(v) \hookrightarrow \mathcal{T}_{C_{\mathfrak{g}}(v)}(k)$.

\section{TORI AND SEMISIMPLE ELEMENTS}

Let $\mathfrak{t} \subset \mathfrak{g}$ be a maximal torus of the finite-dimensional restricted Lie algebra $(\mathfrak{g},[p])$. The main result of this section, Theorem [3.5, asserts that $\mathfrak{t}$ is "generically contained" in a torus of dimension $\mu(\mathfrak{g})$ in the sense that such an inclusion holds on a dense open subset of $\mathcal{X}_{\mathfrak{t}}(k)$. While the results of this section substantially overlap with earlier work by Premet [22], our approach via schemes of tori differs significantly from Premet's methods.

Let $n:=\operatorname{dim}_{k} \mathfrak{g}$. For an element $v \in \mathfrak{g}$ we consider the Fitting-0-space

$$
\mathfrak{g}_{0}(v):=\left\{x \in \mathfrak{g} ;(\operatorname{ad} v)^{n}(x)=0\right\}
$$

of the linear transformation ad $v: \mathfrak{g} \longrightarrow \mathfrak{g} ; x \mapsto[v, x]$. For a subset $V \subset \mathfrak{g}$ we put $\mathfrak{g}_{0}(V):=\bigcap_{v \in V} \mathfrak{g}_{0}(v)$. Since $\mathfrak{g}_{0}(V)=C_{\mathfrak{g}}\left(V^{[p]^{m}}\right)$ for a suitably chosen $m \in \mathbb{N}$, the space $\mathfrak{g}_{0}(V)$ is a $p$-subalgebra of $\mathfrak{g}$. Observe that $\mathfrak{g}_{0}(V)$ is self-normalizing.

An element $a \in \mathfrak{g}$ is said to be regular if the dimension of $\mathfrak{g}_{0}(a)$ is minimal. The set $\operatorname{Reg}(\mathfrak{g})$ of regular elements is a dense open subset of $\mathfrak{g}$. For easy reference we collect a few basic facts concerning Cartan subalgebras and regular elements.

Lemma 3.1. The following statements hold:

(1) For every $a \in \operatorname{Reg}(\mathfrak{g})$ the algebra $\mathfrak{g}_{0}(a)$ is a Cartan subalgebra of $\mathfrak{g}$.

(2) Let $\mathfrak{h} \subset \mathfrak{g}$ be a Cartan subalgebra. Then there exists a maximal torus $\mathfrak{t} \subset \mathfrak{g}$ such that $\mathfrak{h}=C_{\mathfrak{g}}(\mathfrak{t})$.

(3) Let $\mathfrak{h} \subset \mathfrak{g}$ be a Cartan subalgebra. Then there exists an element $a \in \mathfrak{g}$ such that $\mathfrak{h}=\mathfrak{g}_{0}(a)$.

Proof. (1), (2) This can be found in [29, (I.4.7)] and [29, (II.4.1)], respectively.

(3) This was proved in [1, Thm.1]. 
We define the rank of $\mathfrak{g} \operatorname{via} \operatorname{rk}(\mathfrak{g}):=\operatorname{dim}_{k} \mathfrak{g}_{0}(a)$, where $a \in \operatorname{Reg}(\mathfrak{g})$. (This is not to be confused with our earlier notion of the rank of a projective module.)

If $W \subset \mathfrak{g}$ is a subspace, then $W_{p}$ denotes the smallest $[p]$-stable subspace of $\mathfrak{g}$ containing $W$. Thus, $W_{p}=(k x)_{p}=\sum_{i>0} k x^{[p]^{i}}$ in case $W=k x$.

For the reader's convenience we begin by recording two basic facts that will be used repeatedly in the sequel. All topological notions are understood to refer to the Zariski topology of the ambient spaces.

Lemma 3.2. Let $\mathfrak{t} \subset \mathfrak{g}$ be a torus. Then there exists a dense open subset $U \subset \mathfrak{t}$ such that

(a) $\mathfrak{t}=(k t)_{p}$ for every $t \in U$, and

(b) $C_{\mathfrak{g}}(\mathfrak{t})=C_{\mathfrak{g}}(t)$ for every $t \in U$.

Proof. By general theory (cf. [29, (II.3.6)]) there exists a basis $\left\{t_{1}, \ldots, t_{n}\right\}$ of $\mathfrak{t}$ such that $t_{i}^{[p]}=t_{i}$ for $1 \leq i \leq n$. Consider the polynomial $f:=\operatorname{det}\left(\left(X_{i}^{p^{j-1}}\right)_{1 \leq i, j \leq n}\right) \in$ $k\left[X_{1}, \ldots, X_{n}\right]$. If $t=\sum_{i=1} \alpha_{i} t_{i}$ is an element of $\mathfrak{t}$, then $\left\{t, t^{[p]}, \ldots, t^{[p]^{n-1}}\right\}$ is linearly independent if and only if $f\left(\alpha_{1}, \ldots, \alpha_{n}\right) \neq 0$. Since $f \neq 0$, the dense open subset $U:=\left\{\sum_{i=1}^{n} \alpha_{i} t_{i} ; f\left(\alpha_{1}, \ldots, \alpha_{n}\right) \neq 0\right\}$ has property (a).

Let $t$ be an element of $U$. If $x \in C_{\mathfrak{g}}(t)$, then $k t \subset C_{\mathfrak{g}}(x)$. As the latter space is a $p$ subalgebra of $\mathfrak{g}$, it follows that $\mathfrak{t}=(k t)_{p} \subset C_{\mathfrak{g}}(x)$, whence $x \in C_{\mathfrak{g}}(\mathfrak{t})$. Consequently, the set $U$ also satisfies (b).

Recall that an element $s \in \mathfrak{g}$ is referred to as semisimple if $s \in\left(k s^{[p]}\right)_{p}$. Thus, the semisimple elements of $\mathfrak{g}$ are those belonging to some torus. We denote by $S_{\mathfrak{g}}$ the set of semisimple elements of $\mathfrak{g}$. Since ad $s: \mathfrak{g} \longrightarrow \mathfrak{g}$ is a semisimple transformation for every $s \in S_{\mathfrak{g}}$, we have $\mathfrak{g}_{0}(s)=C_{\mathfrak{g}}(s) \forall s \in S_{\mathfrak{g}}$. Note that the set $\operatorname{Regs}(\mathfrak{g}):=S_{\mathfrak{g}} \cap \operatorname{Reg}(\mathfrak{g})$ of regular semisimple elements is an open subset of $S_{\mathfrak{g}}$.

Given an element $x \in \mathfrak{g}$, the Jordan-Chevalley-Seligman decomposition provides a semisimple element $x_{s}$ and a $p$-nilpotent element $x_{n}$ such that

$$
x=x_{s}+x_{n} \text { and }\left[x_{s}, x_{n}\right]=0 .
$$

This implies $\mathfrak{g}_{0}(x)=C_{\mathfrak{g}}\left(x_{s}\right)$, so that $x$ is regular if and only if $x_{s}$ is regular in the sense of [5, Chap. 2, §1].

If the Lie algebra $\mathfrak{g}$ is nilpotent, then $S_{\mathfrak{g}} \subset C(\mathfrak{g})$ is the unique maximal torus of $\mathfrak{g}$ (cf. [29, (II.4.2)]). In view of [29, (II.3.6)] the algebra $\mathfrak{g}$ admits only finitely many tori in this case. Conversely, if $\mathfrak{g}$ possesses only finitely many tori and $\mathfrak{t} \subset \mathfrak{g}$ is a maximal torus, then, the finiteness of the automorphism group of $\mathfrak{t}$ implies $\operatorname{dim} \mathcal{X}_{\mathfrak{t}}=0$, and a consecutive application of [11, (1.6)] and [29, (II.4.1)] shows that $\mathfrak{g}=C_{\mathfrak{g}}(\mathfrak{t})$ is nilpotent. Consequently, the restricted Lie algebra $\mathfrak{g}$ is nilpotent if and only if $\mathcal{T}_{\mathfrak{g}}(k)$ is finite for every $\mathfrak{t} \subset \mathfrak{g}$.

Lemma 3.3. Let $\mathfrak{t} \subset \mathfrak{g}$ be a torus. Then the following statements hold:

(1) $\operatorname{dim}_{k} C_{\mathfrak{g}}(\mathfrak{t}) \geq \operatorname{rk}(\mathfrak{g})$.

(2) $\mathfrak{t} \cap \operatorname{Regs}(\mathfrak{g}) \neq \emptyset$ if and only if $\operatorname{dim}_{k} C_{\mathfrak{g}}(\mathfrak{t})=\operatorname{rk}(\mathfrak{g})$. In that case $C_{\mathfrak{g}}(\mathfrak{t})$ is a Cartan subalgebra of $\mathfrak{g}$, and there exists $t \in \mathfrak{t} \cap \operatorname{Regs}(\mathfrak{g})$ such that $\mathfrak{t}=(k t)_{p}$.

Proof. Thanks to (3.2) we have $C_{\mathfrak{g}}(\mathfrak{t})=C_{\mathfrak{g}}(t)$ for a suitably chosen element $t \in \mathfrak{t}$. Consequently, $\operatorname{dim}_{k} C_{\mathfrak{g}}(\mathfrak{t})=\operatorname{dim}_{k} C_{\mathfrak{g}}(t)=\operatorname{dim}_{k} \mathfrak{g}_{0}(t) \geq \operatorname{rk}(\mathfrak{g})$. Moreover, if equality holds, then $t$ is regular, and $t \in \mathfrak{t} \cap \operatorname{Regs}(\mathfrak{g})$. Now suppose that $\mathfrak{t} \cap \operatorname{Regs}(\mathfrak{g}) \neq \emptyset$. Since $\mathfrak{t} \cap \operatorname{Regs}(\mathfrak{g})=\mathfrak{t} \cap \operatorname{Reg}(\mathfrak{g})$ is an open subset of $\mathfrak{t}$, (3.2) provides an element 
$t \in \mathfrak{t} \cap \operatorname{Regs}(\mathfrak{g})$ such that $C_{\mathfrak{g}}(\mathfrak{t})=C_{\mathfrak{g}}(t)$. As a result, $C_{\mathfrak{g}}(\mathfrak{t})=\mathfrak{g}_{0}(t)$ is a Cartan subalgebra of dimension $\operatorname{rk}(\mathfrak{g})$.

Let $\mathfrak{h} \subset \mathfrak{g}$ be a Cartan subalgebra. According to (3.1(2)) there exists a maximal torus $\mathfrak{t} \subset \mathfrak{g}$ such that $\mathfrak{h}=C_{\mathfrak{g}}(\mathfrak{t})$, and (3.3) yields $\operatorname{dim}_{k} \mathfrak{h} \geq \operatorname{rk}(\mathfrak{g})$. Thus, $\operatorname{rk}(\mathfrak{g})$ is the minimum dimension of all Cartan subalgebras of $\mathfrak{g}$. We will see in $\S 7$ that an arbitrary Cartan subalgebra of $\mathfrak{g}$ always contains a deformation of a Cartan subalgebra of dimension $\operatorname{rk}(\mathfrak{g})$.

Lemma 3.4. Let $\mathfrak{t} \subset \mathfrak{g}$ be a torus, $\mathcal{X} \subset \mathcal{T}_{\mathfrak{g}}$ an irreducible component. If $\varphi_{0} \in \mathcal{X}(k)$ is a rational point such that $\varphi_{0}(\mathfrak{t}) \subset \mathfrak{g}$ is a maximal torus, then $\pi_{\mathfrak{g}}: \mathfrak{g}_{\mathfrak{g}}, \mathcal{X} \longrightarrow \mathfrak{g}$ is a dominant morphism.

Proof. Owing to (3.2) there exists an element $t \in \varphi_{0}(\mathfrak{t})$ such that $\varphi_{0}(\mathfrak{t})=(k t)_{p}$. As $\varphi_{0}(\mathfrak{t})$ is maximal, it follows that the algebra $C_{\mathfrak{g}}\left(\varphi_{0}(\mathfrak{t})\right)=C_{\mathfrak{g}}(t)$ is nilpotent. Thus, by (2.3), the fibre $\pi_{\mathfrak{g}}^{-1}\left(\pi_{\mathfrak{g}}\left(\varphi_{0}, t\right)\right) \subset \mathcal{T}_{C_{\mathfrak{g}}(t)}(k)$ is finite, and a consecutive application of (2.2) and (1.2) yields the assertion.

We are now in a position to establish the generic containment of tori. Parts (1) and (2) of the following result were first obtained by Premet [22, Thm.1, Thm.2(i)], who used generalizations of Winter's exponentials [30].

Theorem 3.5. Let $\mathfrak{t} \subset \mathfrak{g}$ be a maximal torus.

(1) If $\operatorname{dim}_{k} \mathfrak{t}=\mu(\mathfrak{g})$, then $\operatorname{dim}_{k} C_{\mathfrak{g}}(\mathfrak{t})=\operatorname{rk}(\mathfrak{g})$, and there exists $t \in \operatorname{Regs}(\mathfrak{g})$ such that $\mathfrak{t}=(k t)_{p}$.

(2) There exists a dense open subset $U \subset \operatorname{Reg}(\mathfrak{g})$ such that $\mu\left(\mathfrak{g}_{0}(a)\right)=\mu(\mathfrak{g})$ for every $a \in U$.

(3) There exists a dense open subset $O \subset \mathcal{X}_{\mathfrak{t}}(k)$ such that $\mu\left(\tilde{\mathfrak{g}}_{0}(x)\right)=\mu(\mathfrak{g})$ for every $x \in O$.

Proof. (1),(2) Suppose that $\mathfrak{t}$ has dimension $\mu(\mathfrak{g})$. Thanks to (3.4) the morphism $\pi_{\mathfrak{g}}: \mathfrak{g}_{\mathfrak{g}_{0}, \mathcal{X}_{\mathfrak{t}}} \longrightarrow \mathfrak{g}$ is dominant. Accordingly, its image $\operatorname{im} \pi_{\mathfrak{g}}=\bigcup_{x \in \mathcal{X}_{\mathfrak{t}}(k)} \tilde{\mathfrak{g}}_{0}(x)$ contains a dense open subset of $\mathfrak{g}$. Since $\mathfrak{g}$ is irreducible, the intersection $U$ of this open set with $\operatorname{Reg}(\mathfrak{g})$ is not empty, and is thus a dense subset of $\mathfrak{g}$.

Let $a$ be an element of $U$. Then there exists an element $x_{0} \in \mathcal{X}_{\mathfrak{t}}(k)$ such that $a \in$ $\tilde{\mathfrak{g}}_{0}\left(x_{0}\right)$. As $\mathfrak{t}$ has maximal dimension, $\tilde{\mathfrak{g}}_{0}\left(x_{0}\right)=C_{\mathfrak{g}}\left(\varphi_{x_{0}}(\mathfrak{t})\right)$ is a Cartan subalgebra of $\mathfrak{g}$. Consequently, $\tilde{\mathfrak{g}}_{0}\left(x_{0}\right) \subset \mathfrak{g}_{0}(a)$, while (1) of (3.3) implies $\operatorname{dim}_{k} \tilde{\mathfrak{g}}_{0}\left(x_{0}\right) \geq \operatorname{rk}(\mathfrak{g})=$ $\operatorname{dim}_{k} \mathfrak{g}_{0}(a)$. Thus, $\tilde{\mathfrak{g}}_{0}\left(x_{0}\right)=\mathfrak{g}_{0}(a)$, so that

$$
\mu\left(\mathfrak{g}_{0}(a)\right)=\mu\left(\tilde{\mathfrak{g}}_{0}\left(x_{0}\right)\right)=\mu(\mathfrak{g}) .
$$

Since the map $x \mapsto \operatorname{dim}_{k} \tilde{\mathfrak{g}}_{0}(x)$ is constant on $\mathcal{X}_{\mathfrak{t}}(k)$, we also obtain

$$
\operatorname{dim}_{k} C_{\mathfrak{g}}(\mathfrak{t})=\operatorname{dim}_{k} \tilde{\mathfrak{g}}_{0}\left(x_{0}\right)=\operatorname{rk}(\mathfrak{g}),
$$

as claimed. In virtue of (3.3) this implies the existence of $t \in \operatorname{Regs}(\mathfrak{g})$ such that $\mathfrak{t}=(k t)_{p}$.

(3) Now let $\mathfrak{t} \subset \mathfrak{g}$ be an arbitrary maximal torus. Owing to Lemma 3.4 the map $\pi_{\mathfrak{g}}: \mathfrak{g}_{\tilde{\mathfrak{g}}_{0}, \mathcal{X}_{\mathfrak{t}}} \longrightarrow \mathfrak{g}$ is dominant, and its image thereby contains a dense open subset of $\mathfrak{g}$. In particular, $\operatorname{im} \pi_{\mathfrak{g}}$ meets the open set $U$ given in (2). As a result, there exist elements $x_{0} \in \mathcal{X}_{\mathfrak{t}}(k)$ and $p_{0} \in \tilde{\mathfrak{g}}_{0}$ such that $\left(\mathrm{id}_{\mathfrak{g}} \otimes x_{0}\right)\left(p_{0}\right) \in U$. The map

$$
\psi: \mathcal{X}_{\mathfrak{t}}(k) \longrightarrow \mathfrak{g} ; x \mapsto\left(\operatorname{id}_{\mathfrak{g}} \otimes x\right)\left(p_{0}\right)
$$

is a morphism of affine varieties. By the above observation $O:=\psi^{-1}(U)$ is a dense open subset of the irreducible variety $\mathcal{X}_{\mathfrak{t}}(k)$. 
Let $x \in O$. Then $a:=\psi(x)$ is contained in $\tilde{\mathfrak{g}}_{0}(x) \cap U=C_{\mathfrak{g}}\left(\varphi_{x}(\mathfrak{t})\right) \cap U$, so that $\varphi_{x}(\mathfrak{t}) \subset \mathfrak{g}_{0}(a)$. As the element $a$ belongs to $U, \mathfrak{g}_{0}(a)$ is a Cartan subalgebra with $\mu\left(\mathfrak{g}_{0}(a)\right)=\mu(\mathfrak{g})$. Hence there exists a torus $\mathfrak{t}^{\prime}$ of dimension $\mu(\mathfrak{g})$ such that $\varphi_{x}(\mathfrak{t}) \subset \mathfrak{t}^{\prime}$. Consequently, $\mathfrak{t}^{\prime} \subset C_{\mathfrak{g}}\left(\varphi_{x}(\mathfrak{t})\right)=\tilde{\mathfrak{g}}_{0}(x)$, and $\mu\left(\tilde{\mathfrak{g}}_{0}(x)\right)=\mu(\mathfrak{g})$.

Remark. (1) Let $\mathfrak{t} \subset \mathfrak{g}$ be a maximal torus, $O \subset \mathcal{X}_{\mathfrak{t}}(k)$ a dense open subset such that $\mu\left(C_{\mathfrak{g}}(\varphi(\mathfrak{t}))\right)=\mu(\mathfrak{g})$ for every $\varphi \in O$. If $\varphi \in O$, then $\varphi(\mathfrak{t}) \subset C\left(C_{\mathfrak{g}}(\varphi(\mathfrak{t}))\right)$ is contained in a torus $\mathfrak{t}_{\varphi}$ of dimension $\mu(\mathfrak{g})$. Thus, either all tori coming from elements of $\mathcal{X}_{\mathfrak{t}}(k)$ are maximal, or "most" of these tori do not have this property.

(2) Suppose that $\operatorname{dim}_{k} \mathfrak{t}=\mu(\mathfrak{g})$. In view of $(3.5(1))$ and [11, (1.6)] the variety $\mathcal{T}_{\mathfrak{g}}(k)$ is equidimensional. We shall see in $\S 4$ below that the irreducible components of $\mathcal{T}_{\mathfrak{g}}$ are even isomorphic.

We record an immediate consequence that will be generalized in (7.6) below.

Corollary 3.6. Let $\mathfrak{t} \subset \mathfrak{g}$ be a self-centralizing torus. Then $\operatorname{dim}_{k} \mathfrak{t}=\mu(\mathfrak{g})$, and $\varphi(\mathfrak{t})=C_{\mathfrak{g}}(\varphi(\mathfrak{t}))$ for every $\varphi \in \mathcal{X}_{\mathfrak{t}}(k)$.

Proof. Since the maps $x \mapsto \operatorname{dim}_{k} \varphi_{x}(\mathfrak{t})$ and $x \mapsto \operatorname{dim}_{k} \tilde{\mathfrak{g}}_{0}(x)$ are constant on $\mathcal{X}_{\mathfrak{t}}(k)$, it follows that $\tilde{\mathfrak{g}}_{0}(x)=\varphi_{x}(\mathfrak{t})$ for every $x \in \mathcal{X}_{\mathfrak{t}}(k)$. Theorem [3.5) (3) provides an element $x_{0} \in \mathcal{X}_{\mathfrak{t}}(k)$ with $\mu\left(\tilde{\mathfrak{g}}_{0}\left(x_{0}\right)\right)=\mu(\mathfrak{g})$. Consequently, we obtain $\operatorname{dim}_{k} \mathfrak{t}=$ $\operatorname{dim}_{k} \varphi_{x_{0}}(\mathfrak{t})=\mu(\mathfrak{g})$, as desired.

We let

$$
N(\mathfrak{g}):=\left\{x \in \mathfrak{g} ; \exists n \in \mathbb{N} \text { such that } x^{[p]^{n}}=0\right\}
$$

be the conical, affine subvariety of $p$-nilpotent elements, the so-called nilpotent cone of $\mathfrak{g}$. We next employ schemes of tori to give a short proof of [22, Thm.2(iii)].

Proposition 3.7. The set $S_{\mathfrak{g}}$ of semisimple elements of $\mathfrak{g}$ is irreducible, constructible, and of dimension $\operatorname{dim} S_{\mathfrak{g}}=\operatorname{dim}_{k} \mathfrak{g}+\mu(\mathfrak{g})-\operatorname{rk}(\mathfrak{g})$.

Proof. Let $\ell:=\operatorname{dim}_{k} \mathfrak{g}$. We begin by verifying $S_{\mathfrak{g}}=\mathfrak{g}^{[p]^{\ell}}$.

Let $x$ be an arbitrary element of $\mathfrak{g}$. We decompose $x=x_{s}+x_{n}$ into its semisimple and $p$-nilpotent part (cf. [29, (II.3.5)]), and observe that $x_{n}^{[p]^{\ell}}=0$. Since $x_{s}$ and $x_{n}$ commute, we have $x^{[p]^{\ell}}=x_{s}^{[p]^{\ell}} \in S_{\mathfrak{g}}$. Consequently, $\mathfrak{g}^{[p]^{\ell}} \subset S_{\mathfrak{g}}$. Let $s \in S_{\mathfrak{g}}$ be a semisimple element. Then $s \in\left(k s^{[p]}\right)_{p}$, and the $p$-map of $(k s)_{p}$ is surjective. Hence $s \in(k s)_{p}^{[p]^{\ell}} \subset \mathfrak{g}^{[p]^{\ell}}$.

The map $x \mapsto x^{[p]^{\ell}}$ is a morphism of irreducible varieties. Consequently, $S_{\mathfrak{g}}$ is, as the image of an irreducible set, irreducible. Moreover, by Chevalley's theorem [21, (I, $\S 8$, Cor.2)], the set $S_{\mathfrak{g}}$ is constructible. It remains to show that the generic fibre of this morphism has $\operatorname{dimension} \operatorname{rk}(\mathfrak{g})-\mu(\mathfrak{g})$.

Let $\mathfrak{t} \subset \mathfrak{g}$ be a torus of dimension $\mu(\mathfrak{g})$, and consider the morphism $\pi_{\mathfrak{g}}: \mathfrak{g}_{\mathfrak{g}_{0}, \mathcal{X}_{\mathfrak{t}}} \longrightarrow$ $\mathfrak{g}$. According to (3.4) $\pi_{\mathfrak{g}}$ is a dominant morphism of irreducible varieties. By our observations above the morphism $\zeta: \mathfrak{g} \longrightarrow \bar{S}_{\mathfrak{g}} ; x \mapsto x^{[p]^{\ell}}$ is also dominant. Consequently, $\gamma:=\zeta \circ \pi_{\mathfrak{g}}$ enjoys this property as well.

Let $s \in \operatorname{Regs}(\mathfrak{g})$ be given and suppose that $(x, v)$ is an element of the fibre $\gamma^{-1}(s)$. Then $v$ is contained in the Cartan subalgebra $\tilde{\mathfrak{g}}_{0}(x)$ of dimension $\operatorname{rk}(\mathfrak{g})$ (cf. (3.5)), and $v^{[p]^{\ell}}=s$. Thus, $s \in C\left(\tilde{\mathfrak{g}}_{0}(x)\right)$, so that $\tilde{\mathfrak{g}}_{0}(x) \subset C_{\mathfrak{g}}(s)$. Since the element $s$ is regular, equality of dimensions forces $\tilde{\mathfrak{g}}_{0}(x)=C_{\mathfrak{g}}(s)$. Accordingly,

$$
\mu\left(C_{\mathfrak{g}}(s)\right)=\mu(\mathfrak{g}),
$$


and $x$ belongs to the finite set $\mathcal{T}_{C_{\mathfrak{g}}(s)}(k)$. In the nilpotent restricted Lie algebra $C_{\mathfrak{g}}(s)$ there exists a central, semisimple element $t_{0}$ such that $t_{0}^{[p]^{\ell}}=s$. This readily implies that $v$ belongs to the variety $t_{0}+N\left(C_{\mathfrak{g}}(s)\right)$, so that

$$
\{x\} \times\left(t_{0}+N\left(C_{\mathfrak{g}}(s)\right)\right) \subset \gamma^{-1}(s) \subset \mathcal{T}_{C_{\mathfrak{g}}(s)}(k) \times\left(t_{0}+N\left(C_{\mathfrak{g}}(s)\right)\right) .
$$

Let $O \subset \operatorname{im} \gamma$ be a dense open subset of $\bar{S}_{\mathfrak{g}}$. Since $\operatorname{im} \gamma \subset S_{\mathfrak{g}}$, the above observation yields

$$
\operatorname{dim} \gamma^{-1}(s)=\operatorname{dim} N\left(C_{\mathfrak{g}}(s)\right)
$$

for every element of the dense open subset $\operatorname{Reg}(\mathfrak{g}) \cap O$ of $\bar{S}_{\mathfrak{g}}$. For such an element $s$ the Jordan-Chevalley decomposition within the nilpotent Lie algebra $C_{\mathfrak{g}}(s)$ provides a bijective morphism

$$
S_{C_{\mathfrak{g}}(s)} \times N\left(C_{\mathfrak{g}}(s)\right) \longrightarrow C_{\mathfrak{g}}(s) ;(t, n) \mapsto t+n .
$$

In the nilpotent Lie algebra $C_{\mathfrak{g}}(s)$, the set $S_{C_{\mathfrak{g}}(s)}$ is the unique maximal torus, so that $\operatorname{dim} S_{C_{\mathfrak{g}}(s)}=\mu\left(C_{\mathfrak{g}}(s)\right)=\mu(\mathfrak{g})$. Consequently, $\operatorname{dim} N\left(C_{\mathfrak{g}}(s)\right)=\operatorname{dim}_{k} C_{\mathfrak{g}}(s)-$ $\operatorname{dim}_{k} S_{C_{\mathfrak{g}}(s)}=\operatorname{rk}(\mathfrak{g})-\mu\left(C_{\mathfrak{g}}(s)\right)=\operatorname{rk}(\mathfrak{g})-\mu(\mathfrak{g})$, and our assertion now follows directly from (2.2).

Example. Let $\mathfrak{g}=k t \oplus k x,[t, x]=x, t^{[p]}=t, x^{[p]}=0$ be the two-dimensional nonabelian restricted Lie algebra. Direct computation shows that $(\alpha t+\beta x)^{[p]}=$ $\alpha^{p} t+\alpha^{p-1} \beta x$. Since $S_{\mathfrak{g}}=\mathfrak{g}^{[p]^{2}}$, this readily implies

$$
S_{\mathfrak{g}}=\{\alpha t+\beta x ; \alpha \neq 0\} \cup\{0\} \cong((k \backslash\{0\}) \times k) \cup\{(0,0)\} .
$$

As $\mathfrak{g} \backslash S_{\mathfrak{g}}$ contains $\{0\} \times(k \backslash\{0\})$, its closure contains $\{(0,0)\}$. Thus, $S_{\mathfrak{g}}$ is not open, and hence not locally closed.

Corollary 3.8. The following statements are equivalent:

(1) There exists a torus $\mathfrak{t} \subset \mathfrak{g}$ such that $\mathfrak{t}=C_{\mathfrak{g}}(\mathfrak{t})$.

(2) $S_{\mathfrak{g}}$ is a dense subset of $\mathfrak{g}$.

(3) $\operatorname{Regs}(\mathfrak{g})$ is a dense subset of $\mathfrak{g}$.

(4) Every torus of $\mathfrak{g}$ of maximal dimension is self-centralizing.

Proof. (1) $\Rightarrow(2)$. Let $\mathfrak{t} \subset \mathfrak{g}$ be a torus such that $C_{\mathfrak{g}}(\mathfrak{t})=\mathfrak{t}$. Thanks to (3.6) and (3.5)(1)) $\operatorname{dim}_{k} \mathfrak{t}=\mu(\mathfrak{g})=\operatorname{rk}(\mathfrak{g})$, and we have $\operatorname{dim} \overline{S_{\mathfrak{g}}}=\operatorname{dim} S_{\mathfrak{g}}=\operatorname{dim}_{k} \mathfrak{g}$, whence $\mathfrak{g}=\overline{S_{\mathfrak{g}}}$.

$(2) \Rightarrow(3)$. Since $S_{\mathfrak{g}}$ is constructible and dense, it contains a nonempty open subset $U$ of $\mathfrak{g}$. Consequently, $\emptyset \neq U \cap \operatorname{Reg}(\mathfrak{g}) \subset \operatorname{Regs}(\mathfrak{g})$ lies dense in $\mathfrak{g}$.

$(3) \Rightarrow(4)$. Since $\mathfrak{g} \supset \overline{S_{\mathfrak{g}}} \supset \overline{\operatorname{Regs}(\mathfrak{g})}=\mathfrak{g}$, it follows from (3.7) that $\operatorname{rk}(\mathfrak{g})=\mu(\mathfrak{g})$. By (3.5(1)) every torus of dimension $\mu(\mathfrak{g})$ is self-centralizing.

$(4) \Rightarrow(1)$. This is trivial.

\section{ThE SCHEME OF TORI OF MAXIMAL DIMENSION}

In this section we study a geometric quotient of the scheme $\mathcal{T}_{\mathfrak{g}}$ in case $\mathfrak{t} \subset \mathfrak{g}$ is a torus of dimension $\mu(\mathfrak{g})$. Our main result, Theorem 4.1, will yield a refinement of (3.5)(1)), see (4.2) below.

Let $\mathfrak{t}$ be a torus of dimension $r, G_{\mathfrak{t}}:=\mathcal{A U} \mathcal{T}(\mathfrak{t})$ the automorphism scheme of $\mathfrak{t}$, i.e., the $k$-group functor $G_{\mathfrak{t}}: M_{k} \longrightarrow$ Ens that associates to every commutative $k$-algebra $R$ the group

$$
G_{\mathfrak{t}}(R)=\operatorname{Aut}_{p}\left(\mathfrak{t} \otimes_{k} R\right)
$$


of automorphisms of the restricted $R$-Lie algebra $\mathfrak{t} \otimes_{k} R$. Since the group scheme $G_{\mathfrak{t}}$ is algebraic and $G_{\mathfrak{t}}(k) \cong \mathrm{GL}_{r}\left(\mathbb{F}_{p}\right)$ is a finite group, the coordinate ring $k\left[G_{\mathfrak{t}}\right]$ is finitely generated and of Krull dimension 0 . Thus, $G_{\mathfrak{t}}$ is a finite algebraic group. It acts contragrediently on $\mathcal{T}_{\mathfrak{g}}$ via

$$
g \cdot \varphi:=\varphi \circ g^{-1} \quad \forall g \in G_{\mathfrak{t}}(R), \varphi \in \mathcal{T}_{\mathfrak{g}}(R), R \in M_{k} .
$$

As $G_{\mathfrak{t}}$ is a finite algebraic group acting freely on $\mathcal{T}_{\mathfrak{g}}$, the geometric quotient $\hat{\mathcal{T}}_{\mathfrak{g}}:=$ $\mathcal{T}_{\mathfrak{g}} / G_{\mathfrak{t}}$ has the structure of an affine algebraic scheme with function algebra $k\left[\hat{\mathcal{T}}_{\mathfrak{g}}\right]=$ $k\left[\mathcal{T}_{\mathfrak{g}}\right]^{G_{\mathfrak{t}}}$ (cf. [16, (I.5.5(6))]). Owing to [6, (III, $\left.\left.\S 1,1.15\right)\right] \hat{\mathcal{T}}_{\mathfrak{g}}(k)=\mathcal{T}_{\mathfrak{g}}(k) / G_{\mathfrak{t}}(k)$ may be identified with the set $\left\{\mathfrak{t} \in \operatorname{Tor}(\mathfrak{g}) ; \operatorname{dim}_{k} \mathfrak{t}=r\right\}$. The geometric nature of this correspondence will be elaborated on in $\S 8$.

Examples. (1) Let $\mathfrak{g}$ be nilpotent, $\mathfrak{t} \subset \mathfrak{g}$ a torus. Then $\hat{\mathcal{T}}_{\mathfrak{g}}(k)$ is finite, and the variety $\hat{\mathcal{T}}_{\mathfrak{g}}(k)$ is irreducible if and only if $\operatorname{dim}_{k} \mathfrak{t}=\mu(\mathfrak{g})$.

(2) Let $\mathfrak{g}=k t \oplus k x,[t, x]=x, t^{[p]}=t, x^{[p]}=0$, and consider the variety $T_{\mathfrak{g}}:=\left\{s \in \mathfrak{g} ; s^{[p]}=s\right\}$ of toral elements of $\mathfrak{g}$. Since $(\alpha t+\beta x)^{[p]}=\alpha^{p} t+\alpha^{p-1} \beta x$, we have

$$
T_{\mathfrak{g}}=\left\{\alpha t+\beta x ; \alpha \in \mathbb{F}_{p} \backslash\{0\}, \beta \in k\right\} \cup\{0\} .
$$

Now let $\mathfrak{t}=k t$. Then $\mathfrak{t}$ is a torus of maximal dimension, and the map

$$
\mathcal{T}_{\mathfrak{g}}(k) \longrightarrow T_{\mathfrak{g}} \backslash\{0\} \quad ; \quad \varphi \mapsto \varphi(t)
$$

is an isomorphism, so that $\mathcal{T}_{\mathfrak{g}}(k)$ is the disjoint union of $p-1$ copies of $\mathbb{A}^{1}$. The above map also induces an isomorphism $\hat{\mathcal{T}}_{\mathfrak{g}}(k) \longrightarrow \mathbb{A}^{1}$ (cf. also (6.1) below).

Definition. Let $(\mathfrak{g},[p])$ be a finite-dimensional restricted Lie algebra, $\mathfrak{t} \subset \mathfrak{g}$ a torus of maximal dimension. Then $S(\mathfrak{g}, \mathfrak{t}):=\operatorname{Stab}_{G_{\mathfrak{t}}(k)}\left(\mathcal{X}_{\mathfrak{t}}(k)\right)$ is called the toral stabilizer of $\mathfrak{g}$ (relative to $\mathfrak{t}$ ).

Remarks. (1) Since the smooth scheme $\mathcal{X}_{\mathbf{t}}$ is reduced, an application of [16. (I.2.6(11))] shows that $S(\mathfrak{g}, \mathfrak{t})$ is the finite group of rational points of the finite algebraic group $\operatorname{Stab}_{G_{\mathfrak{t}}}\left(\mathcal{X}_{\mathfrak{t}}\right)$.

(2) Let $\iota: \mathfrak{t} \hookrightarrow \mathfrak{g}$ be the given embedding and identify the set $G_{\mathfrak{t}}(k)$ with $G_{\mathfrak{t}}(k) \cdot \iota \subset \mathcal{T}_{\mathfrak{g}}(k)$. As $\mathcal{T}_{\mathfrak{g}}(k)$ is smooth, $\mathcal{X}_{\mathfrak{t}}(k)$ is a connected component of $\mathcal{T}_{\mathfrak{g}}(k)$. Consequently, $g \in G_{\mathfrak{t}}(k)$ belongs to $S(\mathfrak{g}, \mathfrak{t})$ if and only if $\iota \circ g=g^{-1} \cdot \iota \in \mathcal{X}_{\mathfrak{t}}(k)$. Hence we obtain

$$
S(\mathfrak{g}, \mathfrak{t})=G_{\mathfrak{t}}(k) \cap \mathcal{X}_{\mathfrak{t}}(k) .
$$

Theorem 4.1. Let $(\mathfrak{g},[p])$ be a finite-dimensional restricted Lie algebra, $\mathfrak{t} \subset \mathfrak{g}$ a torus of dimension $\mu(\mathfrak{g})$. Then the following statements hold:

(1) $\mathcal{T}_{\mathfrak{g}}(k)=\bigcup_{g \in G_{\mathfrak{t}}(k)} g \cdot \mathcal{X}_{\mathfrak{t}}(k)$, and $\mathcal{T}_{\mathfrak{g}}(k)$ has $\left[G_{\mathfrak{t}}(k): S(\mathfrak{g}, \mathfrak{t})\right]$ irreducible components.

(2) The scheme $\hat{\mathcal{T}}_{\mathfrak{g}}$ is smooth, integral, and of dimension $\operatorname{dim}_{k} \mathfrak{g}-\operatorname{rk}(\mathfrak{g})$.

(3) $\mathcal{X}_{\mathfrak{t}}(k) / S(\mathfrak{g}, \mathfrak{t}) \cong \hat{\mathcal{T}}_{\mathfrak{g}}(k)$.

Proof. (1) Let $\mathcal{X} \subset \mathcal{T}_{\mathfrak{g}}$ be an arbitrary irreducible component, $j: \mathfrak{t} \hookrightarrow \mathfrak{g} \otimes_{k} k[\mathcal{X}]$ the corresponding universal embedding. As before, we consider the canonical projection $\pi_{\mathfrak{g}}: \mathfrak{g}_{k[\mathcal{X}] j(\mathfrak{t}), \mathcal{X}} \longrightarrow \overline{S_{\mathfrak{g}}}$. In view of $[2.2$, (3.5) $(1))$ and (3.7) the varieties $\mathfrak{g}_{k[\mathcal{X}] j(\mathfrak{t}), \mathcal{X}}$ and $\overline{S_{\mathfrak{g}}}$ are irreducible and of equal dimension. 
Let $\varphi$ be an element of $\mathcal{X}(k)$. Since $\mathfrak{t}$ is a torus of maximal dimension, the torus $\varphi(\mathfrak{t})$ also has maximal dimension, and (B.5(1)) implies the existence of an element $t \in \operatorname{Regs}(\mathfrak{g}) \cap \varphi(\mathfrak{t})$. By (2.3) and the nilpotency of $C_{\mathfrak{g}}(t)$ the fibre $\pi_{\mathfrak{g}}^{-1}\left(\pi_{\mathfrak{g}}(\varphi, t)\right)$ is finite, and (1.2) ensures the dominance of the morphism $\pi_{\mathfrak{g}}$. Accordingly, $S_{\mathcal{X}}:=$ $\operatorname{im} \pi_{\mathfrak{g}} \subset S_{\mathfrak{g}}$ contains a dense open subset of $\overline{S_{\mathfrak{g}}}$. Since $\operatorname{Reg}(\mathfrak{g}) \cap \overline{S_{\mathfrak{g}}}$ is a nonempty open subset of the irreducible variety $\overline{S_{\mathfrak{g}}}$, we see that $\emptyset \neq S_{\mathcal{X}} \cap S_{\mathcal{X}_{\mathfrak{t}}} \cap \operatorname{Reg}(\mathfrak{g})=$ $S_{\mathcal{X}} \cap S_{\mathcal{X}_{\mathfrak{t}}} \cap \operatorname{Regs}(\mathfrak{g})$.

Let $t_{0}$ be an element of $S_{\mathcal{X}} \cap S_{\mathcal{X}_{\mathfrak{t}}} \cap \operatorname{Regs}(\mathfrak{g})$. Then there exist rational points $\varphi_{0} \in \mathcal{X}(k)$ and $\psi_{0} \in \mathcal{X}_{\mathfrak{t}}(k)$ such that $t_{0} \in \varphi_{0}(\mathfrak{t}) \cap \psi_{0}(\mathfrak{t})$. For $\varphi \in\left\{\varphi_{0}, \psi_{0}\right\}$ the Cartan subalgebra $C_{\mathfrak{g}}(\varphi(\mathfrak{t}))$ is contained in the nilpotent Lie algebra $C_{\mathfrak{g}}\left(t_{0}\right)$. Consequently, $C_{\mathfrak{g}}(\varphi(\mathfrak{t}))=C_{\mathfrak{g}}\left(t_{0}\right)$, and the maximality of $\varphi(\mathfrak{t})$ implies $\varphi(\mathfrak{t})=S_{\mathfrak{g}} \cap C_{\mathfrak{g}}\left(t_{0}\right)$ for $\varphi \in$ $\left\{\varphi_{0}, \psi_{0}\right\}$. Thus, $g:=\varphi_{0}^{-1} \circ \psi_{0}$ belongs to $G_{\mathfrak{t}}(k)$, and $g \cdot \psi_{0}=\psi_{0} \circ \psi_{0}^{-1} \circ \varphi_{0}=\varphi_{0}$. This shows that $\mathcal{X}(k) \cap g \cdot \mathcal{X}_{\mathfrak{t}}(k) \neq \emptyset$. Owing to [11, (1.4)] the scheme $\mathcal{T}_{\mathfrak{g}}$ is smooth. Hence its irreducible components are its connected components, and $\mathcal{X}(k)=g \cdot \mathcal{X}_{\mathfrak{t}}(k)$.

Let $g, h \in G_{\mathfrak{t}}(k)$ be two elements such that $g \cdot \mathcal{X}_{\mathfrak{t}}(k)=h \cdot \mathcal{X}_{\mathfrak{t}}(k)$. Then $g h^{-1}$ belongs to $S(\mathfrak{g}, \mathfrak{t})$. This completes the proof of (1).

(2) Consider the canonical morphism $\pi_{k}: \mathcal{T}_{\mathfrak{g}}(k) \longrightarrow \hat{\mathcal{T}}_{\mathfrak{g}}(k)$. Thanks to (1), we have $\hat{\mathcal{T}}_{\mathfrak{g}}(k)=\pi_{k}\left(\mathcal{X}_{\mathfrak{t}}(k)\right)$, so that the variety $\hat{\mathcal{T}}_{\mathfrak{g}}(k)$ is irreducible. By (1.3) $\pi_{k}$ is étale and thus sends regular points to regular points. The smoothness of $\hat{\mathcal{T}}_{\mathfrak{g}}(k)$ now follows from [11, (1.4)]. Accordingly, the reduced algebra $k\left[\hat{\mathcal{T}}_{\mathfrak{g}}\right] \subset k\left[\mathcal{T}_{\mathfrak{g}}\right]$ is an integral domain, so that $\hat{\mathcal{T}}_{\mathfrak{g}}$ is integral. Since each fibre of $\left.\pi_{k}\right|_{\mathcal{X}_{\mathfrak{t}}(k)}$ is finite, we have $\operatorname{dim} \hat{\mathcal{T}}_{\mathfrak{g}}(k)=\operatorname{dim} \mathcal{X}_{\mathfrak{t}}(k)=\operatorname{dim}_{k} \mathfrak{g}-\operatorname{rk}(\mathfrak{g})$.

(3) Owing to (1.3) the restriction $h: \mathcal{X}_{\mathfrak{t}}(k) \longrightarrow \hat{\mathcal{T}}_{\mathfrak{g}}(k)$ of $\pi_{k}$ to the connected component $\mathcal{X}_{\mathfrak{t}}(k)$ is étale. According to (1) it induces a bijective morphism

$$
\mathcal{X}_{\mathfrak{t}}(k) / S(\mathfrak{g}, \mathfrak{t}) \longrightarrow \hat{\mathcal{T}}_{\mathfrak{g}}(k),
$$

whose composite with the projection $\mathcal{X}_{\mathfrak{t}}(k) \longrightarrow \mathcal{X}_{\mathfrak{t}}(k) / S(\mathfrak{g}, \mathfrak{t})$ is $h$. Thanks to Lemma 1.4 this map is étale, and [18 (3.6.3.4)] (which states that every étale and universally injective morphism is an open immersion) shows that it is in fact an isomorphism.

Remark. According to (4.1(1)) the stabilizers of the components of $\mathcal{T}_{\mathfrak{g}}(k)$ are conjugate. Hence the structure of $S(\mathfrak{g}, \mathfrak{t})$ does not depend on the choice of $\mathfrak{t}$ and is thus an invariant of the restricted Lie algebra $(\mathfrak{g},[p])$.

Example. Theorem 4.1 does not hold for tori that do not have maximal dimension. Consider the restricted Lie algebra $\mathfrak{g}:=k t \oplus k x \oplus k z$, with Lie bracket $[t, x]=$ $x,[t, z]=0=[x, z]$ and $p$-map $t^{[p]}=t, x^{[p]}=0, z^{[p]}=z$ as well as the variety $\mathcal{T}_{\mathfrak{g}}(k)$ associated to the torus $\mathfrak{t}:=k t$. According to [11, (1.6)] we have $\operatorname{dim} \mathcal{X}_{\mathfrak{t}}=1$ for the component containing the inclusion $\mathfrak{t} \subset \mathfrak{g}$, while the component $\mathcal{Y} \subset \mathcal{T}_{\mathfrak{g}}$ having the embedding $\mathfrak{t} \hookrightarrow \mathfrak{g} ; t \mapsto z$ as a rational point is zero-dimensional. Direct computation shows that $\hat{\mathcal{T}}_{\mathfrak{g}}(k)$ has $p$ irreducible components of dimension one, and one irreducible component of dimension zero.

The general fact underlying our example is the following direct consequence of 11, (1.6)]: Let $\mathfrak{t} \subset \mathfrak{g}$ be a torus. The variety $\mathcal{T}_{\mathfrak{g}}(k)$ has an irreducible component of dimension zero if and only if $\operatorname{dim}_{k} \mathfrak{t} \leq \mu(C(\mathfrak{g}))$.

Our first application, which slightly generalizes [23, Cor.1], concerns the distribution of weight spaces. 
Corollary 4.2. Let $\mathfrak{t}$ and $\mathfrak{t}^{\prime}$ be two tori of $\mathfrak{g}$ of maximal dimension. If $M$ is a restricted $\mathfrak{g}$-module with weight space decompositions $M=\bigoplus_{\lambda \in \Lambda} M_{\lambda}$, and $M=$ $\bigoplus_{\lambda^{\prime} \in \Lambda^{\prime}} M_{\lambda^{\prime}}$ relative to $\mathfrak{t}$ and $\mathfrak{t}^{\prime}$, respectively, then there exists an isomorphism $\varphi$ : $\mathfrak{t} \longrightarrow \mathfrak{t}^{\prime}$ such that $\lambda \mapsto \lambda \circ \varphi^{-1}$ defines a bijection $\Lambda \longrightarrow \Lambda^{\prime}$ satisfying $\operatorname{dim}_{k} M_{\lambda \circ \varphi^{-1}}=$ $\operatorname{dim}_{k} M_{\lambda}$ for every $\lambda \in \Lambda$.

Proof. We put $A:=k\left[\mathcal{X}_{\mathfrak{t}}\right]$, and let

$$
\tilde{M}=\bigoplus_{\gamma \in \Gamma} \tilde{M}_{\gamma}
$$

be the weight space decomposition of $\tilde{M}:=M \otimes_{k} A$ induced by the universal embedding $j: \mathfrak{t} \longrightarrow \tilde{\mathfrak{g}}$ of $\mathcal{X}_{\mathfrak{t}}$. Since $\mathfrak{t}$ and $\mathfrak{t}^{\prime}$ have maximal dimension, Theorem 4.1 provides an element $\varphi \in \mathcal{X}_{\mathfrak{t}}(k)$ such that $\mathfrak{t}^{\prime}=\varphi(\mathfrak{t})$. Let $x_{0} \in \operatorname{Spec}_{k}(A)(k)$ be the corresponding element, so that $\varphi=\left(\mathrm{id}_{\mathfrak{g}} \otimes x_{0}\right) \circ j$. According to our introductory remarks of Section 2, the weight space decomposition of $\mathfrak{g}$ relative to $\mathfrak{t}^{\prime}$ is given by

$$
M=\bigoplus_{\gamma \in \Gamma} \tilde{M}_{\gamma}\left(x_{0}\right)
$$

where $\tilde{M}_{\gamma}\left(x_{0}\right)=M_{\gamma \circ \varphi^{-1}}$ is the weight space relative to $\mathfrak{t}^{\prime}$. By applying the same reasoning to the canonical embedding $\mathfrak{t} \hookrightarrow \mathfrak{g}$ we obtain $\Lambda=\Gamma$, and $\Lambda^{\prime}=\{\lambda \circ$ $\left.\varphi^{-1} ; \lambda \in \Lambda\right\}$. Since the functions $x \mapsto \operatorname{dim}_{k} \tilde{M}_{\gamma}(x)$ are constant on $\mathcal{X}_{\mathfrak{t}}(k)$, we have $\operatorname{dim}_{k} M_{\lambda \circ \varphi^{-1}}=\operatorname{dim}_{k} M_{\lambda}$ for every weight $\lambda \in \Lambda$.

Corollary 4.3. Let $\mathfrak{t}$ and $\mathfrak{t}^{\prime}$ be two tori of $\mathfrak{g}$ of maximal dimension, $\mathfrak{g}=C_{\mathfrak{g}}(\mathfrak{t}) \oplus$ $\bigoplus_{\alpha \in R} \mathfrak{g}_{\alpha}$, and $\mathfrak{g}=C_{\mathfrak{g}}\left(\mathfrak{t}^{\prime}\right) \oplus \bigoplus_{\beta \in R^{\prime}} \mathfrak{g}_{\beta}$ the root space decompositions of $\mathfrak{g}$ relative to $\mathfrak{t}$ and $\mathfrak{t}^{\prime}$, respectively. Then $\operatorname{dim}_{k} C_{\mathfrak{g}}(\mathfrak{t})=\operatorname{dim}_{k} C_{\mathfrak{g}}\left(\mathfrak{t}^{\prime}\right)$, and there exists a bijection $\sigma: R \longrightarrow R^{\prime}$ such that $\operatorname{dim}_{k} \mathfrak{g}_{\sigma(\alpha)}=\operatorname{dim}_{k} \mathfrak{g}_{\alpha}$ for every $\alpha \in R$.

We continue by recovering the conjugacy theorem for maximal tori of restricted Lie algebras of smooth algebraic groups and the characterization via tori of the group (cf. [14, (13.3), (13.5)]).

Corollary 4.4. Let $\mathfrak{g}=\operatorname{Lie}(\mathcal{G})$ be the Lie algebra of a smooth, connected, algebraic $k$-group.

(1) Any two maximal tori and any two Cartan subalgebras of $\mathfrak{g}$ are conjugate.

(2) Given a maximal torus $\mathfrak{t} \subset \mathfrak{g}$, there exists a maximal torus $T \subset \mathcal{G}$ such that $\mathfrak{t}=\operatorname{Lie}(T)$.

Proof. (1) Let $\mathfrak{t}$ be an arbitrary maximal torus of $\mathfrak{g}, \mathfrak{s}_{0} \subset \mathfrak{g}$ a torus of dimension $\mu(\mathfrak{g})$. Thanks to $[3.5(3))$ there exists an element $\varphi \in \mathcal{X}_{\mathfrak{t}}(k)$ such that the torus $\varphi(\mathfrak{t})$ is contained in a torus of dimension $\mu(\mathfrak{g})$. By (2.1) the tori $\mathfrak{t}$ and $\varphi(\mathfrak{t})$ are conjugate, so that $\mathfrak{t}$ is also contained in a torus of dimension $\mu(\mathfrak{g})$. From the maximality of $\mathfrak{t}$ we obtain $\operatorname{dim}_{k} \mathfrak{t}=\mu(\mathfrak{g})$.

According to (4.1(1)) there thus exists an element $\psi \in \mathcal{X}_{\mathfrak{t}}(k)$ such that $\mathfrak{s}_{0}=\psi(\mathfrak{t})$. Another application of (2.1) shows that $\mathfrak{t}$ and $\mathfrak{s}_{0}$ are conjugate, as desired.

Since any Cartan subalgebra of $\mathfrak{g}$ is the centralizer of a maximal torus (3.1), the conjugacy of maximal tori implies the corresponding fact for Cartan subalgebras.

(2) Let $T^{\prime} \subset \mathcal{G}$ be a maximal torus. Thanks to [14, (13.2)] the torus $\mathfrak{t}^{\prime}:=\operatorname{Lie}\left(T^{\prime}\right)$ is maximal. Since $\mathfrak{t}$ and $\mathfrak{t}^{\prime}$ are conjugate, $\mathfrak{t}$ has the required form. 
Example. Suppose that $p \geq 5$, and let $L$ be a simple Lie algebra of Cartan type. Then $L$ contains a subalgebra $\mathfrak{g}$ such that

(a) $\mathfrak{g}$ is a restricted Lie algebra,

(b) there exists a $p$-nilpotent $p$-ideal $\mathfrak{n} \triangleleft \mathfrak{g}$ such that $\mathfrak{g} / \mathfrak{n} \cong \mathfrak{g l}(n), \mathfrak{s l}(n), \mathfrak{s p}(n)$, $\mathfrak{s p}(n) \oplus k z$, where the last algebra is a direct sum of restricted Lie algebras with $z^{[p]}=z$.

In view of (b) and (4.4) any maximal torus of $\mathfrak{g}$ has dimension $\mu(\mathfrak{g})$. Thus, every Cartan subalgebra has dimension $\operatorname{rk}(\mathfrak{g})$ (3.5). Since the roots of $L$ relative to a certain standard maximal torus $\mathfrak{t}_{0} \subset \mathfrak{g}$ form a vector space over the prime field $\mathbb{F}_{p}$ of dimension $\operatorname{dim}_{k} \mathfrak{t}_{0}$, an application of (4.2) shows that this statement obtains for every maximal torus of $\mathfrak{g}$. These facts were established in [2, (2.34)] via switching methods.

We turn to two closely related classes of restricted Lie algebras; classical Lie algebras and Lie algebras of reductive groups. Recall that a restricted Lie algebra $(\mathfrak{g},[p])$ of characteristic $p \geq 5$ is referred to as classical if

(1) $C(\mathfrak{g})=0$ and $[\mathfrak{g}, \mathfrak{g}]=\mathfrak{g}$, and

(2) $\mathfrak{g}$ has an abelian Cartan subalgebra $\mathfrak{h} \subset \mathfrak{g}$ relative to which

(a) $\mathfrak{g}=\mathfrak{h} \oplus \bigoplus_{\alpha \in R} \mathfrak{g}_{\alpha}$, with $\mathfrak{g}_{\alpha}=\{x \in \mathfrak{g} ;[h, x]=\alpha(h) x \quad \forall h \in \mathfrak{h}\}$, and

(b) $\operatorname{dim}_{k}\left[\mathfrak{g}_{\alpha}, \mathfrak{g}_{-\alpha}\right]=1$ for every $\alpha \in R$, and

(c) if $\alpha, \beta \in R$, then there exists $n \in \mathbb{F}_{p}$ such that $\alpha+n \beta \notin R$.

Any Cartan subalgebra of $\mathfrak{g}$ satisfying (a) through (c) is called a classical Cartan subalgebra.

According to 25, (IV.1.2)] all Cartan subalgebras of a classical Lie algebra are conjugate under its automorphism group. Thus, all Cartan subalgebras are classical. Thanks to [25, (II.3.1)] every root $\alpha$ of a Cartan subalgebra $\mathfrak{h} \subset \mathfrak{g}$ gives rise to a unique element $h_{\alpha} \in\left[\mathfrak{g}_{\alpha}, \mathfrak{g}_{-\alpha}\right]$ such that $\alpha\left(h_{\alpha}\right)=2$.

Lemma 4.5. Suppose that $\mathfrak{g}$ is classical with Cartan subalgebra $\mathfrak{h}$. Then we have $h_{\alpha}^{[p]}=h_{\alpha}$ for every $\alpha \in R$. In particular, $\mathfrak{h}$ is a torus of dimension $\mu(\mathfrak{g})$.

Proof. In view of [25. (II.3.2)] the subspace $\mathfrak{g}^{\alpha}:=\mathfrak{g}_{-\alpha} \oplus k h_{\alpha} \oplus \mathfrak{g}_{\alpha}$ is a subalgebra of $\mathfrak{g}$ that is isomorphic to $\mathfrak{s l}(2)$. Moreover, (a) and (c) yield $(\operatorname{ad} e)^{p-1}=0$ for every root vector $e$. Standard $\mathfrak{s l}(2)$ representation theory now implies that the eigenvalues of ad $h_{\alpha}$ lie in the Galois field $\mathbb{F}_{p}$. We conclude that $\beta\left(h_{\alpha}\right) \in \mathbb{F}_{p}$ for every root $\beta \in R$. This yields $\beta\left(h_{\alpha}^{[p]}-h_{\alpha}\right)=\beta\left(h_{\alpha}\right)^{p}-\beta\left(h_{\alpha}\right)=0$ for every $\beta \in R$, so that

$$
h_{\alpha}^{[p]}-h_{\alpha} \in \bigcap_{\beta \in R} \operatorname{ker} \beta=C(\mathfrak{g})=(0),
$$

as desired.

Thanks to axioms (1) and (2), the abelian Lie algebra $\mathfrak{h}$ is generated by the $h_{\alpha}$, proving the surjectivity of the $p$-map on $\mathfrak{h}$. Consequently, the $p$-map is bijective and $\mathfrak{h}$ is a torus. As $\mathfrak{h}$ is self-centralizing, (3.6) implies $\operatorname{dim}_{k} \mathfrak{h}=\mu(\mathfrak{g})$.

Let $\mathfrak{g}$ be a classical Lie algebra, $\mathfrak{t} \subset \mathfrak{g}$ a Cartan subalgebra, $\alpha \in R$ a root. According to Lemma 4.5 the map

$$
s_{\alpha}: \mathfrak{t} \longrightarrow \mathfrak{t} ; t \mapsto t-\alpha(t) h_{\alpha}
$$

belongs to $G_{\mathfrak{t}}(k)$. We define the Weyl group $W(\mathfrak{g}, \mathfrak{t})$ to be the subgroup of $G_{\mathfrak{t}}(k)$ that is generated by $\left\{s_{\alpha} ; \alpha \in R\right\}$. Let $\Sigma \subset R$ be a fundamental system of roots (cf. [25, p. 33]). Following Seligman (cf. [25] p. 45]) we define $W(\mathfrak{g}, \mathfrak{t}, \Sigma)=\left\langle s_{\alpha} ; \alpha \in \Sigma\right\rangle$. 
We consider the canonical action of $G:=\operatorname{Aut}_{p}(\mathfrak{g})^{\circ}$ on $\mathfrak{g}$ along with its induced action on $\mathcal{T}_{\mathfrak{g}}(k)$. We put $\operatorname{Nor}_{G}(\mathfrak{t}):=\{g \in G ; g(\mathfrak{t})=\mathfrak{t}\}$ and $\operatorname{Cent}_{G}(\mathfrak{t}):=\{g \in$ $G ; g(t)=t \forall t \in \mathfrak{t}\}$. Our next result identifies the stabilizer of $\mathcal{X}_{\mathfrak{t}}(k)$.

Theorem 4.6. Let $\mathfrak{t}$ be a Cartan subalgebra of a classical Lie algebra $(\mathfrak{g},[p])$. Then we have $W(\mathfrak{g}, \mathfrak{t})=S(\mathfrak{g}, \mathfrak{t}) \cong \operatorname{Nor}_{G}(\mathfrak{t}) / \operatorname{Cent}_{G}(\mathfrak{t})$.

Proof. According to [25, p. 52f] every element $w \in W(\mathfrak{g}, \mathfrak{t}, \Sigma)$ gives rise to an element $\psi_{w} \in G$ such that $\left.\psi_{w}\right|_{\mathfrak{t}}=w$. We fix a map $w \mapsto \psi_{w}$.

(a) We have $\operatorname{Nor}_{G}(\mathfrak{t})=\left\{\psi_{w} ; w \in W(\mathfrak{g}, \mathfrak{t}, \Sigma)\right\} \operatorname{Cent}_{G}(\mathfrak{t})$.

Set $\mathfrak{g}_{1}(\mathfrak{t}):=\bigoplus_{\alpha \in R} \mathfrak{g}_{\alpha}$. One inclusion being clear, we let $g$ be an element of $\operatorname{Nor}_{G}(\mathfrak{t})$. A consecutive application of [25, (III.5.1)] and the Bruhat-Chevalley decomposition [25. (III.2.1)] provides elements $w \in W(\mathfrak{g}, \mathfrak{t})$, and $v, h, u \in G$ such that $g=v^{-1} \psi_{w} h u$. By definition, the elements $v$ and $u$ are products of exponentials of positive root vectors such that

$$
v(t) \equiv t \equiv u(t) \quad \bmod \mathfrak{g}_{1}(\mathfrak{t}) \quad \forall t \in \mathfrak{t},
$$

while $\left.h\right|_{\mathfrak{t}}=\mathrm{id}_{\mathfrak{t}}$. Let $t$ be an element of $\mathfrak{t}$. Since $g(t) \in \mathfrak{t}$ and $\psi_{w}, h$ permute the root spaces, we obtain

$$
g(t) \equiv v(g(t)) \equiv \psi_{w}(h(u(t))) \equiv \psi_{w}(h(t)) \equiv \psi_{w}(t) \bmod \mathfrak{g}_{1}(\mathfrak{t}),
$$

so that $g(t)=\psi_{w}(t)$. This implies $\left(\psi_{w}\right)^{-1} g \in \operatorname{Cent}_{G}(\mathfrak{t})$, as desired.

(b) We have $W(\mathfrak{g}, \mathfrak{t}) \subset S(\mathfrak{g}, \mathfrak{t})$.

We put $\psi_{\alpha}:=\psi_{s_{\alpha}}$ for $\alpha \in R$. Let $\iota: \mathfrak{t} \hookrightarrow \mathfrak{g}$ be the canonical embedding. Since the orbit $G . \iota$ is connected, we have $G . \iota \subset \mathcal{X}_{\mathfrak{t}}(k)$, whence

$$
s_{\alpha} \cdot \iota=\iota \circ s_{\alpha}=\psi_{\alpha} \circ \iota=\psi_{\alpha} . \iota \in \mathcal{X}_{\mathfrak{t}}(k) .
$$

Thus, $s_{\alpha} \cdot \mathcal{X}_{\mathfrak{t}}(k)=\mathcal{X}_{\mathfrak{t}}(k)$, and our assertion follows.

We continue by showing that $S(\mathfrak{g}, \mathfrak{t}) \subset W(\mathfrak{g}, \mathfrak{t}, \Sigma)$. The connected group $G$ operates on the variety $\mathcal{X}_{\mathfrak{t}}(k)$ in such a way that this action commutes with the one of $S(\mathfrak{g}, \mathfrak{t})$. Accordingly, the map $\varphi \mapsto h \cdot \varphi$ is a $G$-equivariant automorphism of $\mathcal{X}_{\mathfrak{t}}(k)$ for every $h \in S(\mathfrak{g}, \mathfrak{t})$. If $\varphi$ is an element of $\mathcal{X}_{\mathfrak{t}}(k)$, then $\varphi(\mathfrak{t})$ is a classical Cartan subalgebra, and [25, (III.4.1)] provides an element $g \in G$ such that

$$
\varphi(\mathfrak{t})=g(\mathfrak{t})=(g . \iota)(\mathfrak{t}) .
$$

Accordingly, $\varphi \in G$. $(h \cdot \iota)$ for a suitably chosen $h \in S(\mathfrak{g}, \mathfrak{t})$. This shows that the variety $\mathcal{X}_{\mathfrak{t}}(k)$ decomposes into finitely many $G$-orbits, all of which have the same dimension. Since $\mathcal{X}_{\mathfrak{t}}(k)$ is irreducible, one of these orbits must lie dense in $\mathcal{X}_{\mathfrak{t}}(k)$. Consequently, this is true for all of them, and there is only one orbit. It follows that $\mathcal{X}_{\mathbf{t}}(k)=$ G.ı.

Now let $h \in S(\mathfrak{g}, \mathfrak{t})$ be given. Then there exists an element $g \in G$ with $h \cdot \iota=g . \iota$. In particular, $g \in \operatorname{Nor}_{G}(\mathfrak{t})$, and (a) shows that $g=\psi_{w} g^{\prime}$ for some $w \in W(\mathfrak{g}, \mathfrak{t}, \Sigma)$ and $g^{\prime} \in \operatorname{Cent}_{G}(\mathfrak{t})$. Thus,

$$
h \cdot \iota=\left(\psi_{w} g^{\prime}\right) . \iota=\psi_{w} \circ g^{\prime} \circ \iota=\psi_{w} \circ \iota=w^{-1} \cdot \iota,
$$

so that $h=w^{-1} \in W(\mathfrak{g}, \mathfrak{t}, \Sigma)$. Observing (b), we thus obtain

$$
W(\mathfrak{g}, \mathfrak{t}, \Sigma) \subset W(\mathfrak{g}, \mathfrak{t}) \subset S(\mathfrak{g}, \mathfrak{t}) \subset W(\mathfrak{g}, \mathfrak{t}, \Sigma) .
$$

Since $G . \iota=\mathcal{X}_{\mathfrak{t}}(k)$, the canonical restriction map induces a surjective homomorphism $\operatorname{Nor}_{G}(\mathfrak{t}) \longrightarrow S(\mathfrak{g}, \mathfrak{t})$, whose kernel clearly coincides with $\operatorname{Cent}_{G}(\mathfrak{t})$. 
We conclude this section by studying the scheme $\hat{\mathcal{T}}_{\mathfrak{g}}$ for Lie algebras of smooth groups. Given such a group $\mathcal{G}$, and a torus $\mathfrak{t} \subset \mathfrak{g}:=\operatorname{Lie}(\mathcal{G})$, we let $\operatorname{Nor}_{\mathcal{G}}(\mathfrak{t})$ be the normalizer of $\mathfrak{t}$ in $\mathcal{G}$, that is, the subgroup functor given by

$$
\operatorname{Nor}_{\mathcal{G}}(\mathfrak{t})(R):=\left\{g \in \mathcal{G}(R) ; \operatorname{Ad}\left(g_{S}\right)\left(\mathfrak{t} \otimes_{k} S\right)=\mathfrak{t} \otimes_{k} S \text { for all } S \in M_{R}\right\}
$$

for every $R \in M_{k}$. Thanks to [16, (I.2.12(5))] $\operatorname{Nor}_{\mathcal{G}}(\mathfrak{t})$ is a closed subgroup of $\mathcal{G}$. Since the actions of $G_{\mathfrak{t}}$ and $\mathcal{G}$ on $\mathcal{T}_{\mathfrak{g}}$ commute, the universal property of quotient faisceaux provides an operation (cf. [16, (I.5.5(5))])

$$
\mathcal{G} \times \hat{\mathcal{T}}_{\mathfrak{g}} \longrightarrow \hat{\mathcal{T}}_{\mathfrak{g}} .
$$

Let $X: M_{k} \longrightarrow$ Ens be the $k$-functor given by $R \mapsto \mathcal{T}_{\mathfrak{g}}(R) / G_{\mathfrak{t}}(R)$. Then $\hat{\mathcal{T}}_{\mathfrak{g}}$ is the faisceau associated to $X$, and $\mathcal{G}$ operates on $\hat{\mathcal{T}}_{\mathfrak{g}}$ in such a way that the natural transformation $\eta: X \longrightarrow \hat{\mathcal{T}}_{\mathfrak{g}}$ is $\mathcal{G}$-equivariant. According to [16, (I.5.4, I.5.5)] each map $\eta_{R}: X(R) \longrightarrow \hat{\mathcal{T}}_{\mathfrak{g}}(R)$ is injective. Moreover, $\eta_{k}$ is an isomorphism (cf. [6] (III, $\S 1,1.15)])$. Given $\varphi \in \mathcal{T}_{\mathfrak{g}}(k)$, we denote by $[\varphi]$ and $\widehat{[\varphi]}$ the corresponding elements of $X(k)$ and $\hat{\mathcal{T}}_{\mathfrak{g}}(k)$, respectively. Since the image of $[\varphi]$ in $X(R)$ is sent via $\eta_{R}$ onto the image of $\widehat{[\varphi]}$ in $\hat{\mathcal{T}}_{\mathfrak{g}}(R)$, we have

$$
\operatorname{Nor}_{\mathcal{G}}(\varphi(\mathfrak{t}))=\operatorname{Stab}_{\mathcal{G}}([\varphi])=\operatorname{Stab}_{\mathcal{G}}(\widehat{[\varphi]}) \text {. }
$$

Given a smooth, connected, affine algebraic group $\mathcal{G}$ we recall that the correspondence $T \mapsto \operatorname{Lie}(T)$ is a bijection between the sets of maximal tori of $\mathcal{G}(k)$ and $\operatorname{Lie}(\mathcal{G})$ [14, (13.2), (13.3)]. If $T \subset \mathcal{G}(k)$ is a maximal torus, then $W(\mathcal{G}, T):=$ $\operatorname{Nor}_{\mathcal{G}(k)}(T) / \operatorname{Cent}_{\mathcal{G}(k)}(T)$ denotes the Weyl group of $\mathcal{G}$ relative to $T$.

Theorem 4.7. Let $\mathfrak{g}=\operatorname{Lie}(\mathcal{G})$ be the Lie algebra of a smooth, connected, algebraic $k$-group, $\mathfrak{t}=\operatorname{Lie}(T)$ a torus of dimension $\mu(\mathfrak{g})$.

(1) There exists an isomorphism $\mathcal{G} / \operatorname{Nor}_{\mathcal{G}}(\mathfrak{t}) \cong \hat{\mathcal{T}}_{\mathfrak{g}}$ of schemes.

(2) $S(\mathfrak{g}, \mathfrak{t}) \cong \operatorname{Nor}_{\mathcal{G}(k)}(\mathfrak{t}) / \operatorname{Cent}_{\mathcal{G}(k)}(\mathfrak{t})$.

(3) If $p \geq 5$, then $S(\mathfrak{g}, \mathfrak{t}) \cong W(\mathcal{G}, T)$.

Proof. (1) Let $\iota_{0}: \mathfrak{t} \hookrightarrow \mathfrak{g}$ be the canonical embedding. The operation of $\mathcal{G}$ on $\hat{\mathcal{T}}_{\mathfrak{g}}$ induces a morphism

$$
\zeta: \mathcal{G} \longrightarrow \hat{\mathcal{T}}_{\mathfrak{g}} \quad ; g \mapsto g \cdot \widehat{\left[\iota_{0}\right]}
$$

which, in view of the above observation, factors through to a map

$$
\bar{\zeta}: \mathcal{G} / \operatorname{Nor}_{\mathcal{G}}(\mathfrak{t}) \longrightarrow \hat{\mathcal{T}}_{\mathfrak{g}}
$$

It follows from (2.1) and (4.1) that the map $\zeta_{k}$ is a surjective, equivariant morphism with surjective differential $\left(d \zeta_{k}\right)_{1}$. Accordingly,

$$
\bar{\zeta}_{k}: \mathcal{G}(k) / \operatorname{Nor}_{\mathcal{G}(k)}(\mathfrak{t}) \longrightarrow \hat{\mathcal{T}}_{\mathfrak{g}}(k)
$$

is a bijective, $\mathcal{G}(k)$-equivariant morphism whose differential at $\overline{1}$ is surjective. Since the automorphism group of $\mathfrak{t}$ is finite, the connected component $\operatorname{Nor}_{\mathcal{G}(k)}(\mathfrak{t})^{\circ}$ of $\operatorname{Nor}_{\mathcal{G}(k)}(\mathfrak{t})$ is contained in $\operatorname{Cent}_{\mathcal{G}(k)}(\mathfrak{t})$. Hence

$$
\operatorname{dim} \mathcal{G}(k) / \operatorname{Nor}_{\mathcal{G}(k)}(\mathfrak{t})=\operatorname{dim} \mathcal{G}(k)-\operatorname{dim}_{\operatorname{Cent}_{\mathcal{G}(k)}}(\mathfrak{t})=\operatorname{dim}_{k} \mathfrak{g}-\operatorname{rk}(\mathfrak{g})=\operatorname{dim} \hat{\mathcal{T}}_{\mathfrak{g}}(k),
$$

so that $\left(d \bar{\zeta}_{k}\right)_{\overline{1}}$ is in fact an isomorphism. Thanks to [28, (4.3.3)] this implies that $\bar{\zeta}_{k}$ is an isomorphism. Since $\mathcal{G} / \operatorname{Nor}_{\mathcal{G}}(\mathfrak{t})$ and $\hat{\mathcal{T}}_{\mathfrak{g}}$ are schemes, we conclude that $\bar{\zeta}$ is the desired isomorphism (cf. [6, (I, $\S 3,6.8)]$ ). 
(2) Consider the homomorphism

$$
f: \operatorname{Nor}_{\mathcal{G}(k)}(\mathfrak{t}) \longrightarrow G_{\mathfrak{t}}(k) ;\left.h \mapsto \operatorname{Ad}(h)\right|_{\mathfrak{t}}
$$

of groups. Thanks to 2.1 the image of $f$ is contained in $S(\mathfrak{g}, \mathfrak{t})$. If $g$ is an arbitrary element of the latter group, then the product $g \cdot \iota_{0}$ is contained in $\mathcal{X}_{\mathfrak{t}}(k)$. According to 2.1) there exists an element $h \in \mathcal{G}(k)$ with $g \cdot \iota_{0}=\operatorname{Ad}(h) \circ \iota_{0}$. Thus, $h \in \operatorname{Nor}_{\mathcal{G}(k)}(\mathfrak{t})$, and the image of $f$ coincides with $S(\mathfrak{g}, \mathfrak{t})$. The assertion now follows from the equality $\operatorname{ker} f=\operatorname{Cent}_{\mathcal{G}(k)}(\mathfrak{t})$.

(3) Under the assumption that $\mathcal{G}$ is reductive, we first show that the canonical morphism $W(\mathcal{G}, T) \longrightarrow \operatorname{Nor}_{\mathcal{G}(k)}(\mathfrak{t}) / \mathrm{Cent}_{\mathcal{G}(k)}(\mathfrak{t})$ is surjective. It obviously suffices to verify $\operatorname{Nor}_{\mathcal{G}(k)}(\mathfrak{t}) \subset \operatorname{Nor}_{\mathcal{G}(k)}(T) \operatorname{Cent}_{\mathcal{G}(k)}(\mathfrak{t})$. To that end, we let $B \supset T$ be a Borel subgroup of $\mathcal{G}(k)$ and $N \subset \operatorname{Nor}_{\mathcal{G}(k)}(T)$ a complete set of representatives of the Weyl group $W(\mathcal{G}, T)$. There results the Bruhat decomposition

$$
\mathcal{G}=\bigcup_{n \in N} B n B
$$

of $\mathcal{G}$ (cf. [4, (14.12)]).

Let $g$ be an element of $\operatorname{Nor}_{\mathcal{G}(k)}(\mathfrak{t})$. Then there exist $b_{1}, b_{2} \in B$ and $n \in N$ such that $g=b_{1} n b_{2}$. Letting $\mathfrak{g}_{1}(T):=\bigoplus_{\alpha \in R} \mathfrak{g}_{\alpha}$ be the sum of the root spaces relative to $T$, we have

$$
b_{i} . t \equiv t \quad \bmod \mathfrak{g}_{1}(T) \quad \forall t \in \mathfrak{t}, 1 \leq i \leq 2 .
$$

Let $t \in \mathfrak{t}$. Observing $g . t \in \mathfrak{t}$ as well as $n \cdot \mathfrak{g}_{1}(T)=\mathfrak{g}_{1}(T)$, we thus obtain

$$
g . t \equiv b_{1}^{-1} \cdot(g . t) \equiv\left(n b_{2}\right) . t \equiv n . t \quad \bmod \mathfrak{g}_{1}(T)
$$

so that $g . t=n . t$. Consequently, $n^{-1} g \in \operatorname{Cent}_{\mathcal{G}(k)}(\mathfrak{t})$, and $g \in n \operatorname{Cent}_{\mathcal{G}(k)}(\mathfrak{t}) \subset$ $\operatorname{Nor}_{\mathcal{G}(k)}(T) \operatorname{Cent}_{\mathcal{G}(k)}(\mathfrak{t})$.

We now claim that $\operatorname{Cent}_{\mathcal{G}_{(k)}}(T)=\operatorname{Cent}_{\mathcal{G}_{(k)}}(\mathfrak{t})$. One inclusion being obvious, we consider an element $g \in \operatorname{Cent}_{\mathcal{G}(k)}(\mathfrak{t})$. As before, we may write $g=b_{1} n b_{2}$ with $b_{i} \in B$ and $n \in N$. The above reasoning then shows that $n \in \operatorname{Cent}_{\mathcal{G}(k)}(\mathfrak{t})$. Thanks to [14, (3.7)] the root space decomposition of $\mathfrak{g}$ relative to $\mathfrak{t}$ is given by $\mathfrak{g}=\mathfrak{t} \oplus \bigoplus_{\alpha \in R} \mathfrak{g}_{d(\alpha)}$, where $d(\alpha) \in \mathfrak{t}^{*}$ is the differential of the root $\alpha$. Moreover, [14. (5.2)] yields $\mathfrak{g}_{\alpha}=\mathfrak{g}_{d(\alpha)}$ for every $\alpha \in R$. By the above, the element $n$ sends $\mathfrak{g}_{d(\alpha)}$ onto itself. Consequently, the element $w \in W(\mathcal{G}, T)$ corresponding to $n$ induces the identity on $R$. This implies $w=1$, so that $n \in \operatorname{Cent}_{\mathcal{G}(k)}(T)=T$. Hence $g \in B$, and $\operatorname{Cent}_{\mathcal{G}(k)}(\mathfrak{t})=\operatorname{Cent}_{B}(\mathfrak{t})$. The latter group is a closed, $T$-stable subgroup of $B$ containing $T$. In view of $4,(14.4)]$ it is thus directly spanned by $T$ and the root subgroups $U_{\alpha}$ with $\mathfrak{g}_{\alpha} \subset \operatorname{Lie}\left(\operatorname{Cent}_{B}(\mathfrak{t})\right) \subset C_{\operatorname{Lie}(B)}(\mathfrak{t})$. Consequently, $\operatorname{Cent}_{\mathcal{G}(k)}(\mathfrak{t})=$ $\operatorname{Cent}_{B}(\mathfrak{t})=T$, as desired.

As an upshot of the above, the canonical homomorphism $W(\mathcal{G}, T) \longrightarrow \operatorname{Nor}_{\mathcal{G}(k)}(\mathfrak{t}) /$ $\operatorname{Cent}_{\mathcal{G}(k)}(\mathfrak{t})$ is an isomorphism, so that $(2)$ implies our assertion in case $\mathcal{G}$ is reductive.

Now suppose $\mathcal{G}$ is arbitrary, and let $\mathcal{U} \subset \mathcal{G}$ be the unipotent radical of $\mathcal{G}$. We put $\mathcal{G}^{\prime}:=\mathcal{G} / \mathcal{U}$ and denote by $\pi: \mathcal{G} \longrightarrow \mathcal{G}^{\prime}$ the canonical projection. Then $\mathcal{G}^{\prime}$ is reductive and $T^{\prime}:=\pi(T)$ is a maximal torus of $\mathcal{G}^{\prime}$ (cf. [4, (11.14)]). Moreover, $\mathfrak{g}^{\prime}:=\mathfrak{g} / \operatorname{Lie}(\mathcal{U})$ is the Lie algebra of $\mathcal{G}^{\prime}$ with maximal torus $\mathfrak{t}^{\prime}:=\operatorname{Lie}\left(T^{\prime}\right)$ (cf. [4] (6.7)]). Let $\mathcal{X}_{\mathfrak{t}^{\prime}} \subset \mathcal{T}_{\mathfrak{g}^{\prime}}$ be the irreducible component containing the inclusion $\mathfrak{t}^{\prime} \subset \mathfrak{g}^{\prime}$. Since $\operatorname{Lie}(\mathcal{U})$ is $p$-nilpotent, the canonical projection $d \pi: \mathfrak{g} \longrightarrow \mathfrak{g}^{\prime}$ induces an injective homomorphism

$$
\eta: S(\mathfrak{g}, \mathfrak{t}) \longrightarrow S\left(\mathfrak{g}^{\prime}, \mathfrak{t}^{\prime}\right)
$$


such that $\eta(g) \circ d \pi=d \pi \circ g$ for every $g \in G_{\mathfrak{t}}(k)$ (cf. also (5.6)) below. Since $\operatorname{Ad}^{\prime}(\pi(h)) \circ d \pi=d \pi \circ \operatorname{Ad}(h)$ for all $h \in \mathcal{G}(k)$, we obtain a commutative diagram

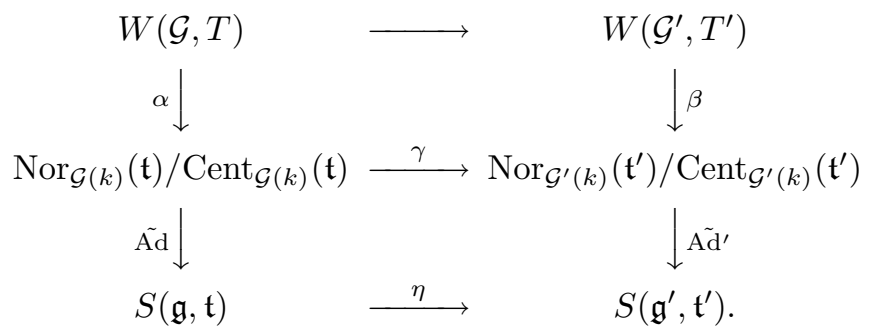

By (2) the maps $\tilde{\mathrm{Ad}}$ and $\tilde{\mathrm{Ad}}{ }^{\prime}$ are isomorphisms, so that the lower square yields the injectivity of $\gamma$. According to [4, (11.20)] and the first part of our proof, the top horizontal map and $\beta$ are isomorphisms. As a result, $\gamma$ is surjective. Hence $\gamma$ is bijective, and $\alpha$ is also an isomorphism.

Remark. (1) The proof of (3) in conjunction with (2) also shows that the morphism $\operatorname{Nor}_{\mathcal{G}(k)}(T) \longrightarrow G_{\mathfrak{t}}(k) ;\left.g \mapsto \operatorname{Ad}(g)\right|_{\mathfrak{t}}$ induces a surjection $W(\mathcal{G}, T) \longrightarrow S(\mathfrak{g}, \mathfrak{t})$ for any $p>0$. Moreover, since for $p \geq 3$ the root space decomposition of a reductive group of semisimple rank 1 coincides with the one of its Lie algebra, the above arguments also imply that $S(\mathfrak{g}, \mathfrak{t})$ is not trivial whenever $\mathcal{G}$ is not solvable.

(2) For $p=2$ the Lie algebra $\mathfrak{g}=\mathfrak{s l}(2)$ of $\mathcal{G}=\mathrm{SL}(2)$ is nilpotent, so that the toral stabilizer is trivial, while the corresponding Weyl group is $\mathbb{Z} /(2)$.

Example. For $p \geq 3$ we consider $\mathcal{G}=\operatorname{SL}(2)$ with its standard torus $T$. Let $\mathfrak{g}=\mathfrak{s l}(2)$ be the Lie algebra of $\mathcal{G}$, and put $\mathfrak{t}:=\operatorname{Lie}(T)$ as well as $t:=\left(\begin{array}{cc}1 & 0 \\ 0 & -1\end{array}\right)$. If $U:=\left\{x \in \mathfrak{g} \backslash\{0\} ; x^{[p]}=x\right\}$, then

$$
\mathcal{T}_{\mathfrak{g}}(k) \longrightarrow U \quad ; \varphi \mapsto \varphi(t)
$$

is a $\mathcal{G}(k)$-equivarant isomorphism. According to (2.1) the connected components of $U$ are the $\mathcal{G}(k)$-orbits, hence the elements of a given component $V \subset U$ have the same eigenvalues and the same determinant. Since each orbit is represented by $\left(\begin{array}{cc}\alpha & 0 \\ 0 & -\alpha\end{array}\right)$ with $\alpha \in \mathbb{F}_{p} \backslash\{0\}$, and

$$
\left(\begin{array}{cc}
0 & 1 \\
-1 & 0
\end{array}\right)\left(\begin{array}{cc}
\alpha & 0 \\
0 & -\alpha
\end{array}\right)\left(\begin{array}{cc}
0 & -1 \\
1 & 0
\end{array}\right)=\left(\begin{array}{cc}
-\alpha & 0 \\
0 & \alpha
\end{array}\right)
$$

we see that $U$ possesses exactly $\frac{p-1}{2}$ components parametrized by $\left\{\alpha^{2} ; \alpha \in \mathbb{F}_{p} \backslash\right.$ $\{0\}$ \}. In particular, the component $V_{0}$ corresponding to $\mathcal{X}_{\mathfrak{t}}(k)$ is given by

$$
V_{0}:=\left\{A \in \mathfrak{g} ; A^{2}=I_{2}\right\},
$$

so that $V_{0}$ is isomorphic to $W_{0}:=\left\{(a, b, c) \in k^{3} ; a^{2}+b c-1=0\right\}$.

According to (4.7(3)), which in our context also holds for $p=3$, the stabilizer of $V_{0} \cong \mathcal{X}_{\mathfrak{t}}(k)$ is generated by the residue class of the matrix $\left(\begin{array}{cc}0 & 1 \\ -1 & 0\end{array}\right)$. Its operation on $W_{0}$ is induced by the automorphism

$$
(a, b, c) \mapsto(-a,-c,-b)
$$

of $\mathbb{A}^{3}$. Consider the automorphism

$$
\tau: \mathbb{A}^{3} \longrightarrow \mathbb{A}^{3} ;(x, y, z) \mapsto(x, y-z, y+z) .
$$


Then

$$
W:=\tau^{-1}\left(W_{0}\right)=\left\{(x, y, z) \in k^{3} ; x^{2}+y^{2}-z^{2}-1=0\right\},
$$

and the operation on $W$ is effected by the transformation

$$
\sigma: \mathbb{A}^{3} \longrightarrow \mathbb{A}^{3} ;(x, y, z) \mapsto(-x,-y, z) .
$$

Let $G$ be the group generated by the comorphism

$$
\gamma: k[X, Y, Z] \longrightarrow k[X, Y, Z] ; X \mapsto-X, Y \mapsto-Y, Z \mapsto Z
$$

of $\sigma$. Since ideal $I=\left(X^{2}+Y^{2}-Z^{2}-1\right)$ of the variety $W$ is generated by a $G$-invariant element, $G$ naturally operates on $k[W]$. According to (4.1) the coordinate ring of $\hat{\mathcal{T}}_{\mathfrak{g}}(k)$ is isomorphic to the algebra $k[W]^{G}$ of invariants. As $p \geq 3$, Maschke's Theorem implies that the canonical projection $k[X, Y, Z] \longrightarrow k[W]$ induces an isomorphism $k[X, Y, Z]^{G} / I^{G} \cong k[W]^{G}$ of $k$-algebras. Since $I$ is generated by the invariant $f:=X^{2}+Y^{2}-Z^{2}-1$, we have $I^{G}=k[X, Y, Z]^{G} f$. Direct computation shows $k[X, Y, Z]^{G}=k\left[X^{2}, Y^{2}, X Y, Z\right]$. Using Gröbner basis techniques the morphism

$$
A \mapsto X^{2}, B \mapsto Y^{2}, C \mapsto X Y, D \mapsto Z
$$

is seen to induce an isomorphism $k[A, B, C, D] /\left(A B-C^{2}\right) \cong k\left[X^{2}, Y^{2}, X Y, Z\right]$. Consequently, this map also induces an isomorphism

$$
k[A, B, C, D] /\left(A+B-D^{2}-1, A B-C^{2}\right) \cong k[W]^{G},
$$

so that we have an isomorphism

$$
\hat{\mathcal{T}}_{\mathfrak{g}}(k) \cong\left\{(a, b, c, d) \in k^{4} ; a+b-d^{2}-1=0, a b-c^{2}=0\right\} .
$$

We shall see later (cf. (6.5), (6.7)) that neither $\mathcal{X}_{\mathfrak{t}}(k)$ nor $\hat{\mathcal{T}}_{\mathfrak{g}}(k)$ is isomorphic to some $\mathbb{A}^{n}$.

\section{The Passage From $\mathfrak{g}$ TO $\mathfrak{g} / \mathfrak{n}$}

5.1. General facts. Let $L$ be a restricted Lie algebra over a commutative $k$-algebra $R$. A $p$-ideal $N \triangleleft L$ is called strongly abelian if $[N, N]=\{0\}=N^{[p]}$. In that case, every element $x \in N$ defines an automorphism

$$
\exp (x): L \longrightarrow L ; y \mapsto y+[x, y]
$$

of restricted $R$-Lie algebras whose composite $\pi \circ \exp (x)$ with the canonical projection $\pi: L \longrightarrow L / N$ coincides with $\pi$.

Throughout this subsection we fix a finite-dimensional torus $(\mathfrak{t},[p])$ over $k$.

Lemma 5.1. Let $L$ be a restricted Lie algebra over a commutative $k$-algebra $R$, $N \triangleleft L$ a strongly abelian $p$-ideal. If $\varphi, \varphi^{\prime}: \mathfrak{t} \longrightarrow L$ are homomorphisms of restricted Lie algebras over $k$ such that $\pi \circ \varphi=\pi \circ \varphi^{\prime}$, then there exists an element $x \in N$ such that

$$
\varphi^{\prime}=\exp (x) \circ \varphi
$$

Proof. We put $\psi:=\pi \circ \varphi$. Since $N$ is abelian, the adjoint action of $L$ on $N$ factors through to an action $L / N \longrightarrow \mathfrak{g l}(N)$. Pullback along $\psi$ gives $N$ the structure of a restricted $\mathrm{t}$-module. The $k$-linear map

$$
\gamma: \mathfrak{t} \longrightarrow N \quad ; \quad t \mapsto \varphi(t)-\varphi^{\prime}(t)
$$

satisfies

$$
s . \gamma(t)-t . \gamma(s)=0 \quad \forall s, t \in \mathfrak{t} .
$$


Moreover, since $N$ is strongly abelian, we have

$$
\begin{aligned}
\varphi\left(t^{[p]}\right) & =\varphi(t)^{[p]}=\left(\varphi^{\prime}(t)+\gamma(t)\right)^{[p]}=\varphi^{\prime}(t)^{[p]}+\left(\operatorname{ad} \varphi^{\prime}(t)\right)^{p-1}(\gamma(t)) \\
& =\varphi^{\prime}\left(t^{[p]}\right)+t^{p-1} \cdot \gamma(t),
\end{aligned}
$$

implying that $\gamma$ is a $p$-derivation. As $\mathfrak{t}$ is a torus, the restricted enveloping algebra $u(\mathfrak{t})$ is semisimple, so that the restricted cohomology group $H_{*}^{1}(\mathfrak{t}, N)=$ $\operatorname{Ext}_{u(\mathfrak{t})}^{1}(k, N)$ vanishes. Thanks to [13, (2.1)] this implies that $\gamma$ is an inner derivation. Accordingly, there exists an element $x \in N$ with $\gamma(t)=t$. $x$ for every $t \in \mathfrak{t}$, whence

$$
\varphi^{\prime}(t)=\varphi(t)-\gamma(t)=\varphi(t)-t . x=\varphi(t)-[\varphi(t), x]=(\exp (x) \circ \varphi)(t),
$$

as desired.

We fix a finite-dimensional restricted Lie algebra $(\mathfrak{g},[p])$ over $k$, and a $p$-ideal $\mathfrak{n} \triangleleft \mathfrak{g}$. Let $\operatorname{Lie}_{p}(\mathfrak{t}, \mathfrak{g})$ be the $k$-functor that assigns to every commutative $k$-algebra $R \in M_{k}$ the set of homomorphisms $\mathfrak{t} \longrightarrow \mathfrak{g} \otimes_{k} R$ of restricted Lie algebras over $k$. The canonical projection $\pi: \mathfrak{g} \longrightarrow \mathfrak{g} / \mathfrak{n}$ induces a morphism

$$
\Pi: \mathcal{T}_{\mathfrak{g}} \longrightarrow \operatorname{Lie}_{p}(\mathfrak{t}, \mathfrak{g} / \mathfrak{n})
$$

such that $\Pi_{R}(\varphi)=\left(\pi \otimes \operatorname{id}_{R}\right) \circ \varphi$ for $\varphi \in \mathcal{T}_{\mathfrak{g}}(R)$ and $R \in M_{k}$.

To see that the above morphism affords a comparison between the schemes of embeddings of $\mathfrak{g}$ and $\mathfrak{g} / \mathfrak{n}$, we require the following subsidiary result.

Lemma 5.2. For every irreducible component $\mathcal{X} \subset \mathcal{T}_{\mathfrak{g}}$, there exists a subtorus $\mathfrak{t}_{\mathcal{X}} \subset \mathfrak{t}$ such that $\varphi^{-1}(\mathfrak{n})=\mathfrak{t}_{\mathcal{X}}$ for every element $\varphi \in \mathcal{X}(k)$.

Proof. Since the scheme $\mathcal{T}_{\mathfrak{g}}$ is smooth [11 (1.4)], the $k$-algebra $A:=k[\mathcal{X}]$ is a finitely generated integral domain. We let $j: \mathfrak{t} \longrightarrow \tilde{\mathfrak{g}}$ be the universal embedding of $\mathfrak{t}$ relative to $\mathcal{X}$, and consider the morphism

$$
\varrho: \operatorname{Spec}_{k}(A) \longrightarrow \operatorname{Lie}_{p}(\mathfrak{t}, \mathfrak{g} / \mathfrak{n}) ; x \mapsto\left(\operatorname{id}_{\mathfrak{g} / \mathfrak{n}} \otimes x\right) \circ\left(\pi \otimes \operatorname{id}_{A}\right) \circ j
$$

of affine algebraic schemes. According to [11, (1.3)] there exists a subtorus $\mathfrak{t}_{\mathcal{X}} \subset \mathfrak{t}$ such that

$$
\operatorname{ker} \varrho_{k}(x)=\mathfrak{t}_{\mathcal{X}} \quad \forall x \in \operatorname{Spec}_{k}(A)(k) .
$$

Let $\varphi$ be an element of $\mathcal{X}(k)$. Then there exists $x \in \operatorname{Spec}_{k}(A)(k)$ such that $\varphi=$ $\left(\operatorname{id}_{\mathfrak{g}} \otimes x\right) \circ j$. Since $\pi \circ \varphi=\varrho_{k}(x)$, we have $\operatorname{ker}(\pi \circ \varphi)=\mathfrak{t}_{\mathcal{X}}$, whence $\varphi^{-1}(\mathfrak{n})=\mathfrak{t}_{\mathcal{X}}$.

For a subtorus $\mathfrak{s} \subset \mathfrak{t}$, we let $\mathcal{Y}^{\mathfrak{s}}$ be the union of all components $\mathcal{X} \subset \mathcal{T}_{\mathfrak{g}}$ such that $\mathfrak{t}_{\mathcal{X}}=\mathfrak{s}$. By general theory, there exists a subtorus $\mathfrak{s}^{\prime} \subset \mathfrak{t}$ with $\mathfrak{t}=\mathfrak{s} \oplus \mathfrak{s}^{\prime}$. There results a morphism

$$
\Pi: \mathcal{Y}^{\mathfrak{s}} \longrightarrow \operatorname{Lie}_{p}\left(\mathfrak{s}^{\prime}, \mathfrak{g} / \mathfrak{n}\right)
$$

which sends $\varphi \in \mathcal{Y}^{\mathfrak{s}}(R)$ onto $\left.\left(\pi \otimes \operatorname{id}_{R}\right) \circ \varphi\right|_{\mathfrak{s}^{\prime}}$.

Given a commutative $k$-algebra $A$ and an ideal $I \triangleleft A$, we denote by $\mathrm{D}(I)$ the open subfunctor of $\operatorname{Spec}_{k}(A)$ associated to $I$ (cf. [16, (I.1.5)]). By definition, we have

$$
\mathrm{D}(I)(R):=\left\{x \in \operatorname{Spec}_{k}(A)(R) ; R x(I)=R\right\}
$$

for every $R \in M_{k}$.

Lemma 5.3. The morphism $\Pi$ induces a morphism $\Pi: \mathcal{Y}^{\mathfrak{s}} \longrightarrow \mathcal{T}_{\mathfrak{g} / \mathfrak{n}}$. 
Proof. According to [11, (1.4)] $\mathcal{T}_{\mathfrak{g} / \mathfrak{n}}$ is an open subfunctor of $\operatorname{Lie}_{p}(\mathfrak{t}, \mathfrak{g} / \mathfrak{n})$. In view of [16, $\left(\right.$ I.1.7(2))] the subfunctor $\mathcal{U}:=\Pi^{-1}\left(\mathcal{T}_{\mathfrak{g} / \mathfrak{n}}\right)$ of the algebraic affine scheme $\mathcal{Y}^{\mathfrak{s}}$ inherits this property. Hence there exists an ideal $I \triangleleft k\left[\mathcal{Y}^{\mathfrak{s}}\right]$ such that $\mathcal{U}=\mathrm{D}(I)$. From [16. (I.1.5(5))] we obtain $\mathrm{D}(I)=\mathrm{D}(\sqrt{I})$. Since $k\left[\mathcal{Y}^{\mathfrak{s}}\right]$ is finitely generated and $k$ is algebraically closed, Hilbert's Nullstellensatz and [16. (I.1.5(8))] imply $\sqrt{I}=\bigcap_{\alpha \in \mathcal{Y}^{\mathfrak{s}}(k) \backslash \mathcal{U}(k)}$ ker $\alpha$. Thus, $\mathcal{U}$ is completely determined by $\mathcal{U}(k)$. In other words, we have $\mathcal{Y}^{\mathfrak{s}}=\mathcal{U}$ whenever $\mathcal{U}(k)=\mathcal{Y}^{\mathfrak{s}}(k)$.

Let $\varphi$ be an element of $\mathcal{Y}^{\mathfrak{s}}(k)$. To verify the injectivity of $\Pi_{k}(\varphi)=\left.\pi \circ \varphi\right|_{\mathfrak{s}^{\prime}}$, we let $s$ be an element of $\operatorname{ker}(\pi \circ \varphi) \cap \mathfrak{s}^{\prime}$. Then we have $s \in \varphi^{-1}(\mathfrak{n}) \cap \mathfrak{s}^{\prime} \subset \mathfrak{s} \cap \mathfrak{s}^{\prime}=(0)$, so that $\varphi \in \mathcal{U}(k)$. Consequently, $\mathcal{U}=\mathcal{Y}^{\mathfrak{s}}$, and $\Pi$ factors through the subfunctor $\mathcal{T}_{\mathfrak{g} / \mathfrak{n}}$.

Lemma 5.4. The morphism $\Pi: \mathcal{Y}^{\mathfrak{s}} \longrightarrow \mathcal{T}_{\mathfrak{g} / \mathfrak{n}}$ is smooth.

Proof. Let $C$ be a finite-dimensional $k$-algebra, $I \triangleleft C$ an ideal such that $I^{2}=(0)$. The corresponding projection $C \longrightarrow C / I$ will be denoted pr. Consider $\varphi \in \mathcal{Y}^{\mathfrak{s}}(C / I)$ and $\psi \in \mathcal{T}_{\mathfrak{g} / \mathfrak{n}}(C)$ such that $\Pi_{C / I}(\varphi)=\psi_{C / I}$. Since the scheme $\mathcal{Y}^{\mathfrak{s}}$ is smooth, there exists an element $\gamma \in \mathcal{Y}^{\mathfrak{s}}(C)$ such that

$$
\varphi=\gamma_{C / I}=\left(\operatorname{id}_{\mathfrak{g}} \otimes \operatorname{pr}\right) \circ \gamma .
$$

As $\Pi$ is a morphism, we have

$$
\left(\mathrm{id}_{\mathfrak{g} / \mathfrak{n}} \otimes \operatorname{pr}\right) \circ \Pi_{C}(\gamma)=\Pi_{C / I}\left(\left(\operatorname{id}_{\mathfrak{g}} \otimes \operatorname{pr}\right) \circ \gamma\right)=\Pi_{C / I}(\varphi)=\psi_{C / I}=\left(\mathrm{id}_{\mathfrak{g} / \mathfrak{n}} \otimes \operatorname{pr}\right) \circ \psi
$$

In view of $(\mathfrak{g} / \mathfrak{n}) \otimes_{k} I$ being a strongly abelian $p$-ideal of $(\mathfrak{g} / \mathfrak{n}) \otimes_{k} C$, Lemma 5.1 provides an element $q \in(\mathfrak{g} / \mathfrak{n}) \otimes_{k} I$ such that

$$
\psi=\exp (q) \circ \Pi_{C}(\gamma) .
$$

Let $q^{\prime} \in \mathfrak{g} \otimes_{k} I$ be an element with $\left(\pi \otimes \operatorname{id}_{C}\right)\left(q^{\prime}\right)=q$.

We next show that $\exp \left(q^{\prime}\right) \circ \gamma \in \mathcal{Y}^{\mathfrak{s}}(C)$. Let $j: \mathfrak{t} \longrightarrow \tilde{\mathfrak{g}}$ be the universal embedding of $\mathcal{T}_{\mathfrak{g}}$ and put $A:=k\left[\mathcal{T}_{\mathfrak{g}}\right]$. Since $\mathcal{Y}^{s} \subset \mathcal{T}_{\mathfrak{g}}$ is an open subfunctor, there exists an ideal $J \triangleleft A$ such that $\mathrm{D}(J)$ is the inverse image of $\mathcal{Y}^{\mathfrak{s}}$ under the isomorphism

$$
\operatorname{Spec}_{k}(A) \longrightarrow \mathcal{T}_{\mathfrak{g}} \quad ; \quad x \mapsto\left(\mathrm{id}_{\mathfrak{g}} \otimes x\right) \circ j .
$$

Let $x$ be an element of $\operatorname{Spec}_{k}(C)(k)$. Since $x$ annihilates the nilpotent ideal $I$, we have

$$
\left(\operatorname{id}_{\mathfrak{g}} \otimes x\right) \circ \exp \left(q^{\prime}\right) \circ \gamma=\left(\operatorname{id}_{\mathfrak{g}} \otimes x\right) \circ \gamma \in \mathcal{Y}^{s}(k) .
$$

Thus, writing $\exp \left(q^{\prime}\right) \circ \gamma=\left(\mathrm{id}_{\mathfrak{g}} \otimes y\right) \circ j$ for a suitable $y \in \operatorname{Spec}_{k}(A)(C)$, we obtain $x \circ y \in \mathrm{D}(J)(k)$ for every $x \in \operatorname{Spec}_{k}(C)(k)$. Consequently, $C y(J)$ is not contained in any maximal ideal of $C$, whence $C y(J)=C$, and $y \in \mathrm{D}(J)(C)$. This implies $\exp \left(q^{\prime}\right) \circ \gamma \in \mathcal{Y}^{\mathfrak{s}}(C)$, as desired.

We now obtain

$$
\Pi_{C}\left(\exp \left(q^{\prime}\right) \circ \gamma\right)=\left.\left(\pi \otimes \operatorname{id}_{C}\right) \circ \exp \left(q^{\prime}\right) \circ \gamma\right|_{\mathfrak{s}^{\prime}}=\exp (q) \circ \Pi_{C}(\gamma)=\psi,
$$

as well as

$$
\left(\exp \left(q^{\prime}\right) \circ \gamma\right)_{C / I}=\left(\mathrm{id}_{\mathfrak{g}} \otimes \operatorname{pr}\right) \circ \exp \left(q^{\prime}\right) \circ \gamma=\gamma_{C / I}=\varphi .
$$

Consequently, $\Pi$ satisfies the lifting property, and is thus a smooth morphism. 
5.2. $p$-nilpotent ideals. Throughout this subsection, $\mathfrak{n} \triangleleft \mathfrak{g}$ is assumed to be a $p$ nilpotent $p$-ideal of the finite-dimensional restricted Lie algebra $(\mathfrak{g},[p])$. As before, we fix a finite-dimensional torus $(\mathfrak{t},[p])$. Given an irreducible component $\mathcal{X} \subset \mathcal{T}_{\mathfrak{g}}$, Lemma 5.2 yields $\varphi^{-1}(\mathfrak{n})=(0)$ for every $\varphi \in \mathcal{X}(k)$. According to Lemma 5.4 the canonical projection $\pi: \mathfrak{g} \longrightarrow \mathfrak{g} / \mathfrak{n}$ gives rise to a smooth morphism $\Pi: \mathcal{T}_{\mathfrak{g}} \longrightarrow \mathcal{T}_{\mathfrak{g} / \mathfrak{n}}$, which will turn out to be an iterated vector bundle.

Lemma 5.5. The morphism $\Pi: \mathcal{T}_{\mathfrak{g}} \longrightarrow \mathcal{T}_{\mathfrak{g} / \mathfrak{n}}$ admits a section $\Sigma: \mathcal{T}_{\mathfrak{g} / \mathfrak{n}} \longrightarrow \mathcal{T}_{\mathfrak{g}}$.

Proof. Setting $A:=k\left[\mathcal{T}_{\mathfrak{g} / \mathfrak{n}}\right]$, we consider the universal embedding $j: \mathfrak{t} \hookrightarrow \widetilde{\mathfrak{g} / \mathfrak{n}}$ of the affine scheme $\mathcal{T}_{\mathfrak{g} / \mathfrak{n}}$. Accordingly,

$$
\Gamma: \operatorname{Spec}_{k}(A) \longrightarrow \mathcal{T}_{\mathfrak{g} / \mathfrak{n}} \quad ; \quad x \mapsto\left(\operatorname{id}_{\mathfrak{g} / \mathfrak{n}} \otimes x\right) \circ j
$$

is an isomorphism. The projection $\tilde{\pi}:=\pi \otimes \mathrm{id}_{A}$ induces an exact sequence

$$
(0) \longrightarrow \mathfrak{n} \otimes_{k} A \longrightarrow \tilde{\pi}^{-1}(j(\mathfrak{t})) \longrightarrow j(\mathfrak{t}) \longrightarrow(0)
$$

of restricted Lie algebras over $k$. By virtue of (3.2) there exists an element $v \in$ $\tilde{\pi}^{-1}(j(\mathfrak{t}))$ such that $j(\mathfrak{t})=(k \tilde{\pi}(v))_{p}$. We can thus find a nonzero $p$-polynomial $f$, so that $f(v) \in \mathfrak{n} \otimes_{k} A$. Suppose that $\mathfrak{n}^{[p]^{m}}=(0)$. Then we have $z^{[p]^{m}} \in[\mathfrak{n}, \mathfrak{n}]_{p} \otimes_{k} A$ for every element $z \in \mathfrak{n} \otimes_{k} A$, and induction on $\operatorname{dim}_{k} \mathfrak{n}$ provides a natural number $\ell \in \mathbb{N}$ such that $\left(\mathfrak{n} \otimes_{k} A\right)^{[p]^{\ell}}=(0)$. It follows that $(k v)_{p}$ is finite-dimensional. By choosing a maximal torus $\mathfrak{s} \subset(k v)_{p}$ we see that the exact sequence

$$
(0) \longrightarrow\left(\mathfrak{n} \otimes_{k} A\right) \cap(k v)_{p} \longrightarrow(k v)_{p} \longrightarrow j(\mathfrak{t}) \longrightarrow(0)
$$

splits. Hence there exists a homomorphism $\omega: j(\mathfrak{t}) \longrightarrow \mathfrak{g} \otimes_{k} A$ of restricted $k$-Lie algebras such that

$$
\tilde{\pi} \circ \omega \circ j=j .
$$

Let $\widetilde{\omega \circ j}: \mathfrak{t} \otimes_{k} A \longrightarrow \mathfrak{g} \otimes_{k} A$ and $\tilde{j}: \mathfrak{t} \otimes_{k} A \longrightarrow \mathfrak{g} \otimes_{k} A$ be the homomorphisms of restricted $A$-Lie algebras corresponding to $\omega \circ j$ and $j$, respectively. Since $j \in$ $\mathcal{T}_{\mathfrak{g} / \mathfrak{n}}(A)$ there exists an $A$-linear map $\tilde{\gamma}: \widetilde{\mathfrak{g} / \mathfrak{n}} \longrightarrow \mathfrak{t} \otimes_{k} A$ such that

$$
\tilde{\gamma} \circ \tilde{j}=\operatorname{id}_{\mathfrak{t} \otimes_{k} A} \text {. }
$$

The above identity gives rise to $\tilde{\pi} \circ \widetilde{\omega \circ j}=\tilde{j}$, whence

$$
\tilde{\gamma} \circ \tilde{\pi} \circ \widetilde{\omega \circ j}=\tilde{\gamma} \circ \tilde{j}=\mathrm{id}_{\mathfrak{t} \otimes_{k} A},
$$

proving that $\omega \circ j \in \mathcal{T}_{\mathfrak{g}}(A)$. Accordingly,

$$
\Phi: \operatorname{Spec}_{k}(A) \longrightarrow \mathcal{T}_{\mathfrak{g}} ; \quad x \mapsto\left(\operatorname{id}_{\mathfrak{g}} \otimes x\right) \circ \omega \circ j
$$

is a morphism of affine schemes. We set $\Sigma:=\Phi \circ \Gamma^{-1}$ and consider, for $R \in M_{k}$ and $x \in \operatorname{Spec}_{k}(A)(R)$, the element $\psi=\Gamma_{R}(x)=\left(\operatorname{id}_{\mathfrak{g} / \mathfrak{n}} \otimes x\right) \circ j$ of $\mathcal{T}_{\mathfrak{g} / \mathfrak{n}}(R)$. Then we have

$$
\begin{aligned}
\Pi_{R}\left(\Sigma_{R}(\psi)\right) & =\left(\pi \otimes \operatorname{id}_{R}\right) \circ \Phi_{R}(x)=\left(\pi \otimes \operatorname{id}_{R}\right) \circ\left(\operatorname{id}_{\mathfrak{g}} \otimes x\right) \circ \omega \circ j=(\pi \otimes x) \circ \omega \circ j \\
& =\left(\operatorname{id}_{\mathfrak{g} / \mathfrak{n}} \otimes x\right) \circ \tilde{\pi} \circ \omega \circ j=\left(\operatorname{id}_{\mathfrak{g} / \mathfrak{n}} \otimes x\right) \circ j=\psi,
\end{aligned}
$$

so that $\Pi \circ \Sigma=\operatorname{id}_{\mathcal{T}_{\mathfrak{g} / \mathfrak{n}}}$.

Suppose that $\mathfrak{n} \subset \mathcal{V}_{C(\mathfrak{g})}$. In view of (5.1) the map $\Pi_{R}$ is injective for every $R \in M_{k}$. Thus, $\Pi$ is an isomorphism in that case. 
If the ideal $\mathfrak{n}$ is strongly abelian, then $\mathfrak{n} \otimes_{k} R$ is strongly abelian for every $R \in M_{k}$, and there results a morphism

$$
\exp : \mathfrak{n} \longrightarrow \mathcal{A U T}(\mathfrak{g}) \quad ; \quad n \mapsto \exp (n)
$$

of affine group schemes. In particular, $\exp _{k}: \mathfrak{n} \longrightarrow \operatorname{Aut}_{p}(\mathfrak{g})$ is a homomorphism of algebraic groups. Since $\mathfrak{n}$ is connected, we have $\exp _{k}(\mathfrak{n}) \subset \operatorname{Aut}_{p}(\mathfrak{g})^{\circ}$.

The following result corresponds to the invariance of the Weyl group under passage to the reductive factor group.

Proposition 5.6. Let $\mathfrak{t} \subset \mathfrak{g}$ be a torus of dimension $\mu(\mathfrak{g})$. Then we have $S(\mathfrak{g}, \mathfrak{t}) \cong$ $S(\mathfrak{g} / \mathfrak{n}, \mathfrak{t})$.

Proof. We proceed by induction on $\operatorname{dim}_{k} \mathfrak{n}$. Assuming $\mathfrak{n}$ to be strongly abelian, we put $\mathfrak{g}^{\prime}:=\mathfrak{g} / \mathfrak{n}$. Since the $G_{\mathfrak{t}}(k)$-equivariant morphism $\Pi_{k}$ sends $\mathcal{X}_{\mathfrak{t}}(k)$ to the component $\mathcal{X}_{\mathfrak{t}}^{\prime}(k)$ of $\mathcal{T}_{\mathfrak{g}^{\prime}}(k)$ containing the inclusion $\left.\pi\right|_{\mathfrak{t}}$, we readily obtain $S(\mathfrak{g}, \mathfrak{t}) \subset$ $S\left(\mathfrak{g}^{\prime}, \mathfrak{t}\right)$.

According to 5 (5.5) there exists a section $\Sigma_{k}^{\prime}: \mathcal{T}_{\mathfrak{g}^{\prime}}(k) \longrightarrow \mathcal{T}_{\mathfrak{g}}(k)$ of $\Pi_{k}$. Owing to $4.1(1))$ we can find $g \in G_{\mathfrak{t}}(k)$ such that $\Sigma_{k}^{\prime}\left(\mathcal{X}_{\mathfrak{t}}^{\prime}(k)\right) \subset g \cdot \mathcal{X}_{\mathfrak{t}}(k)$. Application of $\Pi_{k}$ yields $\mathcal{X}_{\mathfrak{t}}^{\prime}(k) \subset g \cdot \mathcal{X}_{\mathfrak{t}}^{\prime}(k)$. We have equality for dimension reasons, so that $g \in S\left(\mathfrak{g}^{\prime}, \mathfrak{t}\right)$. Consequently, the section

$$
\Sigma_{k}: \mathcal{T}_{\mathfrak{g}^{\prime}}(k) \longrightarrow \mathcal{T}_{\mathfrak{g}}(k) \quad ; \quad \psi \mapsto g^{-1} \cdot \Sigma_{k}^{\prime}(g \cdot \psi)
$$

sends $\mathcal{X}_{\mathfrak{t}}^{\prime}(k)$ to $\mathcal{X}_{\mathfrak{t}}(k)$.

Let $h$ be an element of $S\left(\mathfrak{g}^{\prime}, \mathfrak{t}\right)$. Then we have

$$
\Pi_{k}\left(h \cdot \Sigma_{k}\left(\left.\pi\right|_{\mathfrak{t}}\right)\right)=\left.h \cdot \pi\right|_{\mathfrak{t}}=\Pi_{k}\left(\Sigma_{k}\left(\left.h \cdot \pi\right|_{\mathfrak{t}}\right)\right),
$$

and Lemma 5.1 provides $x \in \mathfrak{n}$ with

$$
h \cdot \Sigma_{k}\left(\left.\pi\right|_{\mathfrak{t}}\right)=\exp _{k}(x) \circ \Sigma_{k}\left(\left.h \cdot \pi\right|_{\mathfrak{t}}\right) .
$$

Since $h \in S\left(\mathfrak{g}^{\prime}, \mathfrak{t}\right)$ and $\exp _{k}(\mathfrak{n}) \subset \operatorname{Aut}_{p}(\mathfrak{g})^{\circ}$, the right-hand side is contained in $\mathcal{X}_{\mathfrak{t}}(k)$. The left-hand side belongs to $h \cdot \mathcal{X}_{\mathfrak{t}}(k)$, so that $\left(h \cdot \mathcal{X}_{\mathfrak{t}}(k)\right) \cap \mathcal{X}_{\mathfrak{t}}(k) \neq \emptyset$. As $\mathcal{T}_{\mathfrak{g}}(k)$ is smooth [11, (1.4)], its irreducible components are its connected components. Hence $\left(h \cdot \mathcal{X}_{\mathfrak{t}}(k)\right)=\mathcal{X}_{\mathfrak{t}}(k)$ and $h$ is contained in $S(\mathfrak{g}, \mathfrak{t})$.

Now let $\mathfrak{n}$ be arbitrary. Then there exists a strongly abelian ideal $(0) \neq \mathfrak{n}_{0} \subset \mathfrak{n}$ of $\mathfrak{g}$. We consider $\mathfrak{g}^{\prime}:=\mathfrak{g} / \mathfrak{n}_{0}$ as well as the $p$-nilpotent ideal $\mathfrak{n}^{\prime}:=\mathfrak{n} / \mathfrak{n}_{0}$ of $\mathfrak{g}^{\prime}$. Thanks to [12, (3.3)] we have $\mu(\mathfrak{g})=\mu\left(\mathfrak{g}^{\prime}\right)$. Consequently, the above in conjuction with the inductive hypothesis yields

$$
S(\mathfrak{g}, \mathfrak{t}) \cong S\left(\mathfrak{g}^{\prime}, \mathfrak{t}\right) \cong S\left(\mathfrak{g}^{\prime} / \mathfrak{n}^{\prime}, \mathfrak{t}\right) \cong S(\mathfrak{g} / \mathfrak{n}, \mathfrak{t})
$$

as desired.

Our next result implies that the smooth morphism $\Pi_{k}: \mathcal{T}_{\mathfrak{g}}(k) \longrightarrow \mathcal{T}_{\mathfrak{g} / \mathfrak{n}}(k)$ is an iterated vector bundle. The reader is referred to [19, Chap. 1] or [27, Chap. VI] concerning basic properties of vector bundles. The Grassmann variety of $\ell$ dimensional subspaces of a finite-dimensional $k$-vector space $V$ will be denoted $\operatorname{Gr}_{\ell}(V)(k)$.

Proposition 5.7. Let $\mathfrak{t} \subset \mathfrak{g}$ be a torus of dimension $\mu(\mathfrak{g})$, and suppose that $\mathfrak{n} \triangleleft \mathfrak{g}$ is strongly abelian. Then $\Pi_{k}: \mathcal{T}_{\mathfrak{g}}(k) \longrightarrow \mathcal{T}_{\mathfrak{g} / \mathfrak{n}}(k)$ is an algebraic vector bundle of $\operatorname{rank} \operatorname{dim}_{k} \mathfrak{n} / C_{\mathfrak{n}}(\mathfrak{t})$. 
Proof. We put $B:=k\left[\mathcal{T}_{\mathfrak{g}}\right]$, and denote by $i: \mathfrak{t} \longrightarrow \tilde{\mathfrak{g}}$ the universal embedding corresponding to the isomorphism $\Delta: \operatorname{Spec}_{k}(B) \longrightarrow \mathcal{T}_{\mathfrak{g}}$. Thanks to (5.5) the morphism $\Pi$ admits a section $\Sigma: \mathcal{T}_{\mathfrak{g} / \mathfrak{n}} \longrightarrow \mathcal{T}_{\mathfrak{g}}$.

As $\mathfrak{n}$ is abelian, the algebra $\mathfrak{g} / \mathfrak{n}$ operates on $\mathfrak{n}$, such that

$$
C_{\mathfrak{n} \otimes_{k} R}\left(\Pi_{R}(\varphi)(\mathfrak{t})\right)=C_{\mathfrak{n} \otimes_{k} R}(\varphi(\mathfrak{t})) \quad \forall R \in M_{k}, \varphi \in \mathcal{T}_{\mathfrak{g}}(R) .
$$

The universal embedding defines, via the weight space decomposition of the $\mathfrak{t}$ module $\mathfrak{n} \otimes_{k} B$, a decomposition

$$
\mathfrak{n} \otimes_{k} B=C_{\mathfrak{n} \otimes_{k} B}(i(\mathfrak{t})) \oplus Q
$$

of $B$-modules.

Since $\mathfrak{n} \otimes_{k} B$ is a strongly abelian ideal, Lemma 5.1 provides, for the elements $i, \Sigma_{B}\left(\Pi_{B}(i)\right) \in \mathcal{T}_{\mathfrak{g}}(B)$, an element $q \in Q$ such that

$$
i=\exp _{B}(q) \circ \Sigma_{B}\left(\Pi_{B}(i)\right) .
$$

Let $\lambda_{q}: \operatorname{Spec}_{k}(B)(k) \longrightarrow \mathfrak{n}$ be the morphism given by $\lambda_{q}(x)=\left(\operatorname{id}_{\mathfrak{g}} \otimes x\right)(q)$, so that $\lambda_{q}(x) \in Q(x)$ for every $x \in \operatorname{Spec}_{k}(B)(k)$. Since $\left(\operatorname{id}_{\mathfrak{g}} \otimes x\right) \circ \exp _{B}(q)=$ $\exp _{k}\left(\lambda_{q}(x)\right) \circ\left(\mathrm{id}_{\mathfrak{g}} \otimes x\right)$ we have

$$
\left(\operatorname{id}_{\mathfrak{g}} \otimes x\right) \circ i=\exp _{k}\left(\lambda_{q}(x)\right) \circ \Sigma_{k}\left(\Pi_{k}\left(\left(\operatorname{id}_{\mathfrak{g}} \otimes x\right) \circ i\right)\right)
$$

for every $x \in \operatorname{Spec}_{k}(B)(k)$. Consequently,

$$
\varphi=\exp _{k}\left(\lambda_{q}\left(\Delta_{k}^{-1}(\varphi)\right)\right) \circ \Sigma_{k}\left(\Pi_{k}(\varphi)\right) \quad \forall \varphi \in \mathcal{T}_{\mathfrak{g}}(k) .
$$

According to Lemma 5.5 the morphism $\Pi_{k}: \mathcal{T}_{\mathfrak{g}}(k) \longrightarrow \mathcal{T}_{\mathfrak{g} / \mathfrak{n}}(k)$ is surjective, and the foregoing identity shows that $\exp _{k}: \mathfrak{n} \longrightarrow \operatorname{Aut}_{p}(\mathfrak{g})$ induces a bijection

$$
\zeta_{\psi}: \mathfrak{n} / C_{\mathfrak{n}}(\psi(\mathfrak{t})) \longrightarrow \Pi_{k}^{-1}(\psi) ; \bar{n} \mapsto \exp _{k}(n) \circ \Sigma_{k}(\psi)
$$

for every $\psi \in \mathcal{T}_{\mathfrak{g} / \mathfrak{n}}(k)$. The maps $\zeta_{\psi}$ endow $\Pi_{k}$ with the structure of a linear fibration.

Since $\operatorname{dim}_{k} \mathfrak{t}=\mu(\mathfrak{g})=\mu(\mathfrak{g} / \mathfrak{n})$ (cf. [12, (3.3)]), Theorem 4.1 implies that the map

$$
\mathcal{T}_{\mathfrak{g} / \mathfrak{n}}(k) \longrightarrow \operatorname{Gr}_{\ell}(\mathfrak{n})(k) ; \psi \mapsto C_{\mathfrak{n}}(\psi(\mathfrak{t})),
$$

with $\ell:=\operatorname{dim}_{k} C_{\mathfrak{n}}(\pi(\mathfrak{t}))$, is a morphism. Accordingly, given $\psi_{0} \in \mathcal{T}_{\mathfrak{g} / \mathfrak{n}}(k)$ and $M_{0} \subset \mathfrak{n}$ such that $C_{\mathfrak{n}}\left(\psi_{0}(\mathfrak{t})\right) \oplus M_{0}=\mathfrak{n}$, the set

$$
U_{0}:=\left\{\psi \in \mathcal{T}_{\mathfrak{g} / \mathfrak{n}}(k) ; C_{\mathfrak{n}}(\psi(\mathfrak{t})) \oplus M_{0}=\mathfrak{n}\right\}
$$

is an open neighbourhood of $\psi_{0}$.

We consider the morphism

$$
\zeta: U_{0} \times M_{0} \longrightarrow \Pi_{k}^{-1}\left(U_{0}\right) ;(\psi, m) \mapsto \exp _{k}(m) \circ \Sigma_{k}(\psi),
$$

and observe that $\Pi_{k} \circ \zeta$ is the projection $U_{0} \times M_{0} \longrightarrow U_{0}$.

We first show that $\zeta$ is injective. Suppose that $(\psi, m),\left(\psi^{\prime}, m^{\prime}\right)$ are elements of $U_{0} \times M_{0}$ with $\zeta(\psi, m)=\zeta\left(\psi^{\prime}, m^{\prime}\right)$. Application of $\Pi_{k}$ implies $\psi=\psi^{\prime}$, whence $\operatorname{ad}\left(m-m^{\prime}\right) \circ \Sigma_{k}(\psi)=0$. Consequently,

$$
m-m^{\prime} \in M_{0} \cap C_{\mathfrak{n}}\left(\Sigma_{k}(\psi)(\mathfrak{t})\right)=M_{0} \cap C_{\mathfrak{n}}(\psi(\mathfrak{t}))=(0),
$$

as desired.

Next, we construct the inverse of $\zeta$. Recall that

$$
U:=\left\{X \in \operatorname{Gr}_{\ell}(\mathfrak{n})(k) ; X \oplus M_{0}=\mathfrak{n}\right\}
$$


is an affine open subset of the Grassmannian such that $U \cong \operatorname{Hom}_{k}\left(\mathfrak{n} / M_{0}, M_{0}\right)$. It follows from this identification that

$$
U \longrightarrow \operatorname{Hom}_{k}\left(\mathfrak{n}, M_{0}\right) \quad ; \quad X \mapsto \operatorname{pr}_{X},
$$

with $\operatorname{pr}_{X}$ being the projection $\mathfrak{n} \longrightarrow M_{0}$ induced by the decomposition $\mathfrak{n}=X \oplus M_{0}$, is a morphism of affine varieties. Given $\psi \in U_{0}$ we set $\operatorname{pr}_{\psi}:=\operatorname{pr}_{C_{\mathfrak{n}}(\psi(\mathfrak{t}))}$. In this fashion we obtain a morphism

$$
\omega: U_{0} \longrightarrow \operatorname{Hom}_{k}\left(\mathfrak{n}, M_{0}\right) \quad ; \quad \psi \mapsto \operatorname{pr}_{\psi} .
$$

We now consider the morphism

$$
\gamma: \Pi_{k}^{-1}\left(U_{0}\right) \longrightarrow U_{0} \times M_{0} \quad ; \quad \varphi \mapsto\left(\Pi_{k}(\varphi), \omega\left(\Pi_{k}(\varphi)\right)\left(\lambda_{q}\left(\Delta_{k}^{-1}(\varphi)\right)\right)\right) .
$$

Given $\psi \in U_{0}$, we have $C_{\mathfrak{n}}(\psi(\mathfrak{t}))=C_{\mathfrak{n}}\left(\Sigma_{k}(\psi)(\mathfrak{t})\right)$, so that

$$
\exp _{k}(\omega(\psi)(n)) \circ \Sigma_{k}(\psi)=\exp _{k}(n) \circ \Sigma_{k}(\psi) \quad \forall n \in \mathfrak{n} .
$$

Observing (11), we now obtain for an element $\varphi \in \Pi_{k}^{-1}\left(U_{0}\right)$

$$
\begin{aligned}
\zeta(\gamma(\varphi)) & =\zeta\left(\Pi_{k}(\varphi), \omega\left(\Pi_{k}(\varphi)\right)\left(\lambda_{q}\left(\Delta_{k}^{-1}(\varphi)\right)\right)\right) \\
& =\exp _{k}\left(\omega\left(\Pi_{k}(\varphi)\right)\left(\lambda_{q}\left(\Delta_{k}^{-1}(\varphi)\right)\right) \circ \Sigma_{k}\left(\Pi_{k}(\varphi)\right)\right. \\
& =\exp _{k}\left(\lambda_{q}\left(\Delta_{k}^{-1}(\varphi)\right)\right) \circ \Sigma_{k}\left(\Pi_{k}(\varphi)\right)=\varphi,
\end{aligned}
$$

so that $\zeta \circ \gamma=\operatorname{id}_{\Pi_{k}^{-1}\left(U_{0}\right)}$. On the other hand, $\zeta$ is injective, and

$$
\zeta \circ(\gamma \circ \zeta)=(\zeta \circ \gamma) \circ \zeta=\zeta
$$

whence $\gamma \circ \zeta=\operatorname{id}_{U_{0} \times M_{0}}$. Accordingly, $\gamma$ and $\zeta$ are isomorphisms. If $p_{U_{0}}: U_{0} \times$ $M_{0} \longrightarrow U_{0}$ denotes the projection, then $p_{U_{0}} \circ \gamma=\Pi_{k}$. It remains to show that, for every $\psi \in U_{0}$, the map $\gamma$ induces an isomorphism $\Pi_{k}^{-1}(\psi) \longrightarrow M_{0}$ of $k$-vector spaces. Let $m$ be an element of $M_{0}$. Then $\zeta(\psi, m)=\exp _{k}(m) \circ \Sigma_{k}(\psi)=\zeta_{\psi}(\bar{m})$ so that the map induced by $\zeta$ corresponds to the isomorphism $M_{0} \longrightarrow \mathfrak{n} / C_{n}(\psi(\mathfrak{t}))$. Consequently, $\gamma$ has the requisite property, and the linear fibration $\Pi_{k}: \mathcal{T}_{\mathfrak{g}}(k) \longrightarrow$ $\mathcal{T}_{\mathfrak{g} / \mathfrak{n}}(k)$ is an algebraic vector bundle of the given rank.

Remark. The interested reader may note that $\mathcal{T}_{\mathfrak{g}} \longrightarrow \mathcal{T}_{\mathfrak{g} / \mathfrak{n}}$ is in fact a schemetheoretic vector bundle.

Let $\Upsilon$ be the set of sections of the $G_{\mathfrak{t}}(k)$-equivariant morphism $\Pi_{k}: \mathcal{T}_{\mathfrak{g}}(k) \longrightarrow$ $\mathcal{T}_{\mathfrak{g} / \mathfrak{n}}(k)$. The group $G_{\mathfrak{t}}(k)$ operates on $\Upsilon$ via

$$
(g \cdot \Sigma)(\psi):=g \Sigma\left(g^{-1} \psi\right) \quad \forall g \in G_{\mathfrak{t}}(k), \Sigma \in \Upsilon, \psi \in \mathcal{T}_{\mathfrak{g} / \mathfrak{n}}(k) .
$$

If $\mathfrak{n} \triangleleft \mathfrak{g}$ is strongly abelian, then $\mathfrak{n}$ acts on $\Upsilon$ via

$$
(n . \Sigma)(\psi):=\exp _{k}(n) \circ \Sigma(\psi) \quad \forall n \in \mathfrak{n}, \Sigma \in \Upsilon, \psi \in \mathcal{T}_{\mathfrak{g} / \mathfrak{n}}(k) .
$$

We collect a few basic properties of these actions.

Lemma 5.8. Let $\mathfrak{n} \triangleleft \mathfrak{g}$ be strongly abelian, $\psi: \mathfrak{t} \hookrightarrow \mathfrak{g} / \mathfrak{n}$ an embedding.

(1) If $\Sigma, \Sigma^{\prime}: \mathcal{T}_{\mathfrak{g} / \mathfrak{n}}(k) \longrightarrow \mathcal{T}_{\mathfrak{g}}(k)$ are sections, then there exists $n \in \mathfrak{n}$ such that

$$
\Sigma^{\prime}(\psi)=(n . \Sigma)(\psi) .
$$

(2) If $n \in \mathfrak{n}$ and $g \in G_{\mathfrak{t}}(k)$, then

$$
(n .(g \cdot \Sigma))(\psi)=(g \cdot(n . \Sigma))(\psi) .
$$


(3) Let $g \in G_{\mathfrak{t}}(k)$. Then there exists $n_{g} \in \mathfrak{n}$ such that

$$
(g \cdot \Sigma)(\psi)=\left(n_{g} \cdot \Sigma\right)(\psi)
$$

for every section $\Sigma: \mathcal{T}_{\mathfrak{g} / \mathfrak{n}}(k) \longrightarrow \mathcal{T}_{\mathfrak{g}}(k)$ of $\Pi_{k}$.

Proof. (1) Let $\pi: \mathfrak{g} \longrightarrow \mathfrak{g} / \mathfrak{n}$ be the canonical projection. Since $\Sigma$ and $\Sigma^{\prime}$ are sections, we have $\pi \circ \Sigma(\psi)=\Pi_{k}(\Sigma(\psi))=\psi=\Pi_{k}\left(\Sigma^{\prime}(\psi)\right)=\pi \circ \Sigma^{\prime}(\psi)$, and Lemma 5.1 yields the assertion.

(2) We have

$$
\begin{aligned}
(n .(g \cdot \Sigma))(\psi) & =\exp (n) \circ\left(g \Sigma\left(g^{-1} \psi\right)\right)=\exp (n) \circ \Sigma\left(g^{-1} \psi\right) \circ g^{-1} \\
& =(n . \Sigma)\left(g^{-1} \psi\right) \circ g^{-1}=g\left((n . \Sigma)\left(g^{-1} \psi\right)\right)=(g \cdot(n . \Sigma))(\psi),
\end{aligned}
$$

as desired.

(3) Let $\Sigma_{0}$ be a section. By (1) there exists $n_{g} \in \mathfrak{n}$ such that

$$
\left(g \cdot \Sigma_{0}\right)(\psi)=\left(n_{g} \cdot \Sigma_{0}\right)(\psi) .
$$

Now let $\Sigma$ be another section. Part (1) provides $m \in \mathfrak{n}$ with

$$
\Sigma(\psi)=\left(m . \Sigma_{0}\right)(\psi) .
$$

In view of Part (2) we have

$$
\begin{aligned}
(g \cdot \Sigma)(\psi) & =\left(g \cdot\left(m \cdot \Sigma_{0}\right)\right)(\psi)=\left(m \cdot\left(g \cdot \Sigma_{0}\right)\right)(\psi)=\exp (m) \circ\left(g \cdot \Sigma_{0}\right)(\psi) \\
& =\exp (m) \circ \exp \left(n_{g}\right) \circ \Sigma_{0}(\psi)=\exp \left(n_{g}\right) \circ \Sigma(\psi),
\end{aligned}
$$

as desired.

Lemma 5.9. Suppose that $\mathfrak{n} \triangleleft \mathfrak{g}$ is strongly abelian, and let $\Sigma: \mathcal{T}_{\mathfrak{g} / \mathfrak{n}}(k) \longrightarrow \mathcal{T}_{\mathfrak{g}}(k)$ be a section of $\Pi_{k}$. If $p \neq 2$ or $\mu(\mathfrak{g}) \neq 2$, then we have

$$
g \cdot \Sigma=\Sigma
$$

for every $g \in G_{\mathfrak{t}}(k)$.

Proof. Let $\Sigma^{\prime}$ be another section, $\psi \in \mathcal{T}_{\mathfrak{g} / \mathfrak{n}}(k)$ an embedding. Since $\mathfrak{n}$ is abelian, we have

$$
C_{\mathfrak{n}}\left(\Sigma^{\prime}(\psi)(\mathfrak{t})\right)=C_{\mathfrak{n}}(\Sigma(\psi)(\mathfrak{t})) .
$$

We choose a subspace $\mathfrak{m} \subset \mathfrak{n}$ with

$$
\mathfrak{n}=\mathfrak{m} \oplus C_{\mathfrak{n}}(\Sigma(\psi)(\mathfrak{t})) .
$$

Given $g \in G_{\mathfrak{t}}(k)$, Lemma $5.8(3)$ now provides a uniquely determined element $m_{g} \in$ $\mathfrak{m}$ such that

$$
(g \cdot \Sigma)(\psi)=\left(m_{g} \cdot \Sigma\right)(\psi) .
$$

We consider the resulting map

$$
\Phi: G_{\mathfrak{t}}(k) \longrightarrow \mathfrak{m} ; g \mapsto m_{g} .
$$

Thanks to $5.8(2),(3))$, and $(*)$ we have

$$
\begin{aligned}
\left(m_{g h} \cdot \Sigma\right)(\psi) & =(g h \cdot \Sigma)(\psi)=(g \cdot(h \cdot \Sigma))(\psi)=\left(m_{g} \cdot(h \cdot \Sigma)\right)(\psi)=\left(h \cdot\left(m_{g} \cdot \Sigma\right)\right)(\psi) \\
& =\left(m_{h} \cdot\left(m_{g} \cdot \Sigma\right)\right)(\psi)=\left(\left(m_{g}+m_{h}\right) \cdot \Sigma\right)(\psi),
\end{aligned}
$$

so that $\Phi: G_{\mathfrak{t}}(k) \longrightarrow \mathfrak{m}$ is a homomorphism of groups. Recall that $G_{\mathfrak{t}}(k) \cong$ $\operatorname{GL}(\mu(\mathfrak{g}))\left(\mathbb{F}_{p}\right)$. Thus, [17, p. 358] implies that, for $p \neq 2,\left(G_{\mathfrak{t}}(k), G_{\mathfrak{t}}(k)\right) \cong$ $\operatorname{SL}(\mu(\mathfrak{g}))\left(\mathbb{F}_{p}\right)$ is a subgroup of index $p-1$. Since $\left(G_{\mathfrak{t}}(k), G_{\mathfrak{t}}(k)\right) \subset \operatorname{ker} \Phi$ and $\mathfrak{m}$ is a $p$-group, it follows that $\Phi(g)=0$ for every $g \in G_{\mathfrak{t}}(k)$. By Lemma 2 of [17] 
p. 358], we have $G_{\mathfrak{t}}(k)=\left(G_{\mathfrak{t}}(k), G_{\mathfrak{t}}(k)\right)$ for $p=2$ and $\mu(\mathfrak{g}) \geq 2$. Since $G_{\mathfrak{t}}(k)=\{1\}$ for $\mu(\mathfrak{g})=1$, we obtain $\Phi(g)=0$ for all $g \in G_{\mathfrak{t}}(k)$ unless $\mu(\mathfrak{g})=2$.

Consequently,

$$
(g \cdot \Sigma)(\psi)=\Sigma(\psi) \quad \forall g \in G_{\mathfrak{t}}(k) .
$$

As $\psi$ was chosen arbitrarily, we obtain $g \cdot \Sigma=\Sigma$ for every $g \in G_{\mathfrak{t}}(k)$.

The $G_{\mathfrak{t}}(k)$-equivariant morphism $\Pi_{k}$ induces a morphism $\hat{\Pi}_{k}: \hat{\mathcal{T}}_{\mathfrak{g}}(k) \longrightarrow \hat{\mathcal{T}}_{\mathfrak{g} / \mathfrak{n}}(k)$. Our next result extends (5.7) to the varieties of schemes of tori of maximal dimension.

Proposition 5.10. Let $\mathfrak{t} \subset \mathfrak{g}$ be a torus of dimension $\mu(\mathfrak{g})$, and suppose that $\mathfrak{n} \triangleleft \mathfrak{g}$ is strongly abelian. If $\mu(\mathfrak{g}) \neq 2$ or $p \neq 2$, then $\hat{\Pi}_{k}: \hat{\mathcal{T}}_{\mathfrak{g}}(k) \longrightarrow \hat{\mathcal{T}}_{\mathfrak{g} / \mathfrak{n}}(k)$ is an algebraic vector bundle of rank $\operatorname{dim}_{k} \mathfrak{n} / C_{\mathfrak{n}}(\mathfrak{t})$.

Proof. Let $\ell:=\operatorname{dim}_{k} C_{\mathfrak{n}}(\mathfrak{t})$. As noted in the proof of (5.7), whose notation we adopt here, the map

$$
\mathcal{T}_{\mathfrak{g} / \mathfrak{n}}(k) \longrightarrow \operatorname{Gr}_{\ell}(\mathfrak{n})(k) \quad ; \quad \psi \mapsto C_{\mathfrak{n}}(\psi(\mathfrak{t}))
$$

is a morphism of varieties. This map factors through to a morphism

$$
\eta: \hat{\mathcal{T}}_{\mathfrak{g}}(k) \longrightarrow \operatorname{Gr}_{\ell}(\mathfrak{n})(k) .
$$

Given $\left[\psi_{0}\right] \in \hat{\mathcal{T}}_{\mathfrak{g}}(k)$, we let $M_{0} \subset \mathfrak{n}$ be a subspace of $\mathfrak{n}$ such that $C_{\mathfrak{n}}\left(\psi_{0}(\mathfrak{t})\right) \oplus M_{0}=\mathfrak{n}$. Then

$$
U:=\left\{X \in \operatorname{Gr}_{\ell}(\mathfrak{n})(k) ; X \oplus M_{0}=\mathfrak{n}\right\}
$$

is an affine open set of $\operatorname{Gr}_{\ell}(\mathfrak{n})(k)$, so that

$$
\hat{U}_{0}:=\left\{[\psi] \in \hat{\mathcal{T}}_{\mathfrak{g} / \mathfrak{n}}(k) ; C_{\mathfrak{n}}(\psi(\mathfrak{t})) \oplus M_{0}=\mathfrak{n}\right\}=\eta^{-1}(U)
$$

is an open neighbourhood of $\left[\psi_{0}\right] \in \hat{\mathcal{T}}_{\mathfrak{g} / \mathfrak{n}}(k)$.

Thanks to (5.5) the $G_{\mathfrak{t}}(k)$-equivariant morphism $\Pi_{k}: \mathcal{T}_{\mathfrak{g}} \longrightarrow \mathcal{T}_{\mathfrak{g} / \mathfrak{n}}$ admits a section $\Sigma: \mathcal{T}_{\mathfrak{g} / \mathfrak{n}}(k) \longrightarrow \mathcal{T}_{\mathfrak{g}}(k)$. In view of Lemma 5.9 the section $\Sigma$ is also $G_{\mathfrak{t}}(k)$ equivariant and thereby induces a morphism

$$
\hat{\Sigma}: \hat{\mathcal{T}}_{\mathfrak{g} / \mathfrak{n}}(k) \longrightarrow \hat{\mathcal{T}}_{\mathfrak{g}}(k) ;[\psi] \mapsto[\Sigma(\psi)] .
$$

In particular, $\hat{\Sigma}$ is a section for the morphism $\hat{\Pi}_{k}: \hat{\mathcal{T}}_{\mathfrak{g}}(k) \longrightarrow \hat{\mathcal{T}}_{\mathfrak{g} / \mathfrak{n}}(k)$.

Given $m \in M_{0}$, we denote by $\exp _{k}(m)$ the automorphism of $\hat{\mathcal{T}}_{\mathfrak{g}}(k)$ defined by

$$
[\psi] \mapsto\left[\exp _{k}(m) \circ \psi\right] .
$$

There results a commutative diagram (cf. 5.7 for the notation)

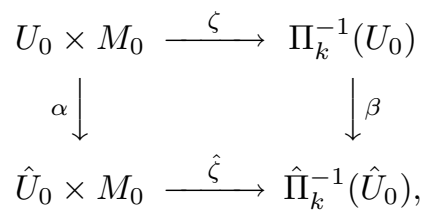

where $\alpha$ and $\beta$ are the canonical maps while $\zeta$ and $\hat{\zeta}$ are defined via

$$
\zeta(\psi, m)=\exp _{k}(m)(\Sigma(\psi)) \text { and } \hat{\zeta}([\psi], m)=\exp _{k}(m)(\hat{\Sigma}([\psi]))
$$

respectively. By the proof of (5.7) $\zeta$ is an isomorphism, so that $\hat{\zeta}$ is a bijective morphism. Since $\beta$ is étale (cf. (1.3)), the same applies to

$$
\beta \circ \zeta=\hat{\zeta} \circ \alpha \text {. }
$$


Observing the faithful flatness of $\alpha$ (cf. (1.3), [20, p. 46]) we may now apply (1.4) to see that $\hat{\zeta}$ is also étale. Consequently, [18, (3.6.3.4)] ensures that $\hat{\zeta}$ is an isomorphism. As a result, $\hat{\Pi}_{k}: \hat{\mathcal{T}}_{\mathfrak{g}}(k) \longrightarrow \hat{\mathcal{T}}_{\mathfrak{g} / \mathfrak{n}}(k)$ is an algebraic vector bundle of the given rank.

Remark. By the proof of the foregoing result $\hat{\Pi}_{k}$ is an algebraic vector bundle whenever $\Pi_{k}$ admits a $G_{\mathfrak{t}}(k)$-equivariant section. This is, for instance, always the case, if there is a homomorphism $\gamma: \mathfrak{g} / \mathfrak{n} \longrightarrow \mathfrak{g}$ of restricted Lie algebras with $\pi \circ \gamma=\mathrm{id}_{\mathfrak{g} / \mathfrak{n}}$.

5.3. Toral ideals. In this subsection we turn to toral $p$-ideals, that is, to $p$-ideals of $\mathfrak{g}$ that are tori. Such ideals are necessarily contained in the center of $\mathfrak{g}$. Given a finite-dimensional torus $(\mathfrak{t},[p])$, we recall that $G_{\mathfrak{t}}(k)=\operatorname{Aut}_{p}(\mathfrak{t})$ denotes the (necessarily finite) automorphism group of $\mathfrak{t}$.

For a decomposition $\mathfrak{t}=\mathfrak{s} \oplus \mathfrak{s}^{\prime}$ of $\mathfrak{t}$ by subtori we consider the subgroup $\operatorname{Nor}_{G_{\mathfrak{t}}(k)}(\mathfrak{s})$ $=\left\{g \in G_{\mathfrak{t}}(k) ; g(\mathfrak{s})=\mathfrak{s}\right\}$ of $G_{\mathfrak{t}}(k)$. If $\operatorname{pr}_{\mathfrak{s}^{\prime}}: \mathfrak{t} \longrightarrow \mathfrak{s}^{\prime}$ and $\operatorname{pr}_{\mathfrak{s}}: \mathfrak{t} \longrightarrow \mathfrak{s}$ are the projections with kernels $\mathfrak{s}$ and $\mathfrak{s}^{\prime}$, respectively, then

$$
\eta: \operatorname{Nor}_{G_{\mathfrak{t}}(k)}(\mathfrak{s}) \longrightarrow G_{\mathfrak{s}^{\prime}}(k) ;\left.g \mapsto\left(\operatorname{pr}_{\mathfrak{s}^{\prime}} \circ g\right)\right|_{\mathfrak{s}^{\prime}}
$$

is readily seen to be a surjective homomorphism of (finite) groups.

Given $\mathfrak{t}=\mathfrak{s} \oplus \mathfrak{s}^{\prime}$ we recall that $\mathcal{Y}^{\mathfrak{s}}$ is the union of those components $\mathcal{X} \subset \mathcal{T}_{\mathfrak{g}}$ such that $\mathfrak{t}_{\mathcal{X}}=\mathfrak{s}$. Note that $\mathcal{Y}^{\mathfrak{s}}(k)$ is a $\operatorname{Nor}_{G_{\mathfrak{t}}(k)}(\mathfrak{s})$-subset of $\mathcal{T}_{\mathfrak{g}}(k)$. If we let $\operatorname{Nor}_{G_{\mathfrak{t}}(k)}(\mathfrak{s})$ operate on $\mathcal{T}_{\mathfrak{g} / \mathfrak{n}}(k)$ via $\eta$, then $\Pi_{k}: \mathcal{Y}^{\mathfrak{s}}(k) \longrightarrow \mathcal{T}_{\mathfrak{g} / \mathfrak{n}}(k)$ is a $\operatorname{Nor}_{G_{\mathfrak{t}}(k)}(\mathfrak{s})$-equivariant morphism.

If $\operatorname{dim}_{k} \mathfrak{t}=\mu(\mathfrak{g})$, and $\mathcal{X}_{\mathfrak{t}} \subset \mathcal{Y}^{\mathfrak{s}}$ (that is, $\left.\mathfrak{s} \subset \mathfrak{n}\right)$, then we have $S(\mathfrak{g}, \mathfrak{t}) \subset \operatorname{Nor}_{G_{\mathfrak{t}}(k)}(\mathfrak{s})$. As $\Pi_{k}\left(\mathcal{X}_{\mathfrak{t}}(k)\right)$ is contained in the component $\mathcal{X}_{\mathfrak{s}^{\prime}}(k)$ containing the embedding $\left.\pi\right|_{\mathfrak{s}^{\prime}}$, we have $\eta(S(\mathfrak{g}, \mathfrak{t})) \subset S\left(\mathfrak{g} / \mathfrak{n}, \mathfrak{s}^{\prime}\right)$. We let $G_{\mathfrak{s}^{\prime}}(k)$ act from the right on the additive group $\operatorname{Lie}_{p}\left(\mathfrak{s}^{\prime}, \mathfrak{s}\right)(k)$, and consider the subgroup $\operatorname{Cent}_{G_{\mathfrak{t}}(k)}(\mathfrak{s}):=\left\{g \in G_{\mathfrak{t}}(k) ;\left.g\right|_{\mathfrak{s}}=\right.$ $\left.\operatorname{id}_{\mathfrak{s}}\right\}$. The map

$$
f_{\mathfrak{s}}: \operatorname{Cent}_{G_{\mathfrak{t}}(k)}(\mathfrak{s}) \longrightarrow G_{\mathfrak{s}^{\prime}}(k) \ltimes \operatorname{Lie}_{p}\left(\mathfrak{s}^{\prime}, \mathfrak{s}\right)(k) \quad ; \quad g \mapsto\left(\eta(g),\left.\operatorname{pr}_{\mathfrak{s}} \circ g\right|_{\mathfrak{s}^{\prime}}\right)
$$

is an embedding of finite groups.

From now on we assume $\mathfrak{n}$ to be a toral $p$-ideal of $\mathfrak{g}$, and let $\mathfrak{t} \subset \mathfrak{g}$ be a torus of dimension $\mu(\mathfrak{g})$. Then $\mathfrak{n}$ is contained in $\varphi(\mathfrak{t})$ for every element $\varphi \in \mathcal{T}_{\mathfrak{g}}(k)$. We pick a subtorus $\mathfrak{s}^{\prime}$ of $\mathfrak{t}$ such that $\mathfrak{t}=\mathfrak{n} \oplus \mathfrak{s}^{\prime}$. Since $\mu(\mathfrak{g})=\mu(\mathfrak{g} / \mathfrak{n})+\operatorname{dim}_{k} \mathfrak{n}$ (cf. [12 (3.3)]), we have $\operatorname{dim}_{k} \mathfrak{s}^{\prime}=\mu(\mathfrak{g} / \mathfrak{n})$.

Lemma 5.11. Let $\mathfrak{n} \triangleleft \mathfrak{g}$ be a toral $p$-ideal, $\mathfrak{t} \subset \mathfrak{g}$ a torus of dimension $\mu(\mathfrak{g})$. If $\mathcal{X} \subset \mathcal{T}_{\mathfrak{g}}$ is an irreducible component, and $\varphi_{0} \in \mathcal{X}(k)$ is a rational point, then $\left.\varphi\right|_{\mathfrak{n}}=\left.\varphi_{0}\right|_{\mathfrak{n}}$ for every $\varphi \in \mathcal{X}(k)$. In particular, we have $S(\mathfrak{g}, \mathfrak{t}) \subset \operatorname{Cent}_{G_{\mathfrak{t}}(k)}(\mathfrak{n})$.

Proof. By (5.2) there exists a subtorus $\mathfrak{t}_{\mathcal{X}} \subset \mathfrak{t}$ with $\varphi^{-1}(\mathfrak{n})=\mathfrak{t}_{\mathcal{X}}$ for every element $\varphi \in \mathcal{X}(k)$. Accordingly, restriction to $\mathfrak{t}_{\mathcal{X}}$ defines a morphism

$$
\mathcal{X}(k) \longrightarrow \operatorname{Lie}_{p}\left(\mathfrak{t}_{\mathcal{X}}, \mathfrak{n}\right)(k) \text {. }
$$

Since $\mathcal{X}(k)$ is connected and $\operatorname{Lie}\left(\mathfrak{t}_{\mathcal{X}}, \mathfrak{n}\right)(k)$ is finite, this map is constant, as desired.

If $g$ is an element of $S(\mathfrak{g}, \mathfrak{t})$, then $\left.\left(g \cdot \varphi_{0}\right)\right|_{\mathfrak{n}}=\left.\varphi_{0}\right|_{\mathfrak{n}}$, so that $\left.g\right|_{\mathfrak{n}}=\operatorname{id}_{\mathfrak{n}}$.

Proposition 5.12. If $\mathfrak{n} \subset \mathfrak{g}$ is a toral $p$-ideal, and $\mathfrak{t}=\mathfrak{n} \oplus \mathfrak{s}^{\prime} \subset \mathfrak{g}$ is a torus of dimension $\mu(\mathfrak{g})$, then the following statements hold:

(1) $\Pi_{k}: \mathcal{Y}^{\mathfrak{n}}(k) \longrightarrow \mathcal{T}_{\mathfrak{g} / \mathfrak{n}}(k) ;\left.\varphi \mapsto(\pi \circ \varphi)\right|_{\mathfrak{s}^{\prime}}$ is a surjective morphism, whose fibres are the $(\operatorname{ker} \eta)$-orbits of $\mathcal{Y}^{\mathfrak{n}}(k)$. 
(2) The homomorphism $\left.\eta\right|_{S(\mathfrak{g}, \mathfrak{t})}: S(\mathfrak{g}, \mathfrak{t}) \longrightarrow S\left(\mathfrak{g} / \mathfrak{n}, \mathfrak{s}^{\prime}\right)$ is surjective.

(3) The homomorphism $f_{\mathfrak{n}}$ induces an embedding

$$
S(\mathfrak{g}, \mathfrak{t}) \hookrightarrow S\left(\mathfrak{g} / \mathfrak{n}, \mathfrak{s}^{\prime}\right) \ltimes \operatorname{Lie}_{p}\left(\mathfrak{s}^{\prime}, \mathfrak{n}\right)(k) .
$$

(4) Let $\mathcal{X}_{\mathfrak{s}^{\prime}}(k)$ be the component of $\mathcal{T}_{\mathfrak{g} / \mathfrak{n}}(k)$ containing $\left.\pi\right|_{\mathfrak{s}^{\prime}}$. Then $\Pi_{k}$ induces isomorphisms $\hat{\mathcal{T}}_{\mathfrak{g}}(k) \cong \hat{\mathcal{T}}_{\mathfrak{g} / \mathfrak{n}}(k)$ and $\mathcal{X}_{\mathfrak{t}}(k) /(\operatorname{ker} \eta \cap S(\mathfrak{g}, \mathfrak{t})) \cong \mathcal{X}_{\mathfrak{s}^{\prime}}(k)$.

Proof. (1) Let $\psi: \mathfrak{s}^{\prime} \longrightarrow \mathfrak{g} / \mathfrak{n}$ be an element of $\mathcal{T}_{\mathfrak{g} / \mathfrak{n}}(k)$. Then $\pi^{-1}\left(\psi\left(\mathfrak{s}^{\prime}\right)\right)$ is, as an extension of tori, a torus of dimension $\mu(\mathfrak{g})$. Hence there exists an element $\lambda \in \mathcal{T}_{\mathfrak{g}}(k)$ such that $(\pi \circ \lambda)(\mathfrak{t})=\psi\left(\mathfrak{s}^{\prime}\right)$. Since $\operatorname{dim}_{k} \lambda^{-1}(\mathfrak{n})=\operatorname{dim}_{k} \mathfrak{n}$, there exists $g \in G_{\mathfrak{t}}(k)$ such that $\gamma:=g \cdot \lambda \in \mathcal{Y}^{\mathfrak{n}}(k)$. It follows that $\psi\left(\mathfrak{s}^{\prime}\right)=(\pi \circ \gamma)(\mathfrak{t})=\Pi_{k}(\gamma)\left(\mathfrak{s}^{\prime}\right)$. Since $\eta$ is surjective, we can find $h \in \operatorname{Nor}_{G_{\mathrm{t}}(k)}(\mathfrak{n})$ such that $\psi=\eta(h) \cdot \Pi_{k}(\gamma)=\Pi_{k}(h \cdot \gamma)$. As $h \cdot \gamma \in \mathcal{Y}^{\mathfrak{n}}(k)$, the surjectivity of $\Pi_{k}$ follows.

Each fibre of the $\operatorname{Nor}_{G_{\mathfrak{t}}(k)}(\mathfrak{n})$-equivariant morphism $\Pi_{k}$ is a (ker $\left.\eta\right)$-subset of $\mathcal{Y}^{\mathfrak{n}}(k)$. Now let $\varphi, \varphi^{\prime} \in \mathcal{Y}^{\mathfrak{n}}(k)$ be given such that $\Pi_{k}(\varphi)=\Pi_{k}\left(\varphi^{\prime}\right)$. Then we have $\pi \circ \varphi=\pi \circ \varphi^{\prime}$, whence $\varphi^{\prime}(\mathfrak{t}) \subset \varphi(\mathfrak{t})+\mathfrak{n} \subset \varphi(\mathfrak{t})$. Thus, there exists an element $g \in G_{\mathfrak{t}}(k)$ such that $\varphi^{\prime}=g \cdot \varphi$. Consequently,

$$
\mathfrak{n}=(g \cdot \varphi)^{-1}(\mathfrak{n})=g\left(\varphi^{-1}(\mathfrak{n})\right)=g(\mathfrak{n}),
$$

so that $g \in \operatorname{Nor}_{G_{\mathfrak{t}}(k)}(\mathfrak{n})$. Moreover, the identity

$$
\Pi_{k}(\varphi)=\Pi_{k}(g \cdot \varphi)=\eta(g) \cdot \Pi_{k}(\varphi)
$$

implies $g \in \operatorname{ker} \eta$.

(2) Let $h$ be an element of $S\left(\mathfrak{g} / \mathfrak{n}, \mathfrak{s}^{\prime}\right)$. Then there exists $g \in \operatorname{Nor}_{G_{\mathfrak{t}}(k)}(\mathfrak{n})$ such that $\eta(g)=h$. Consequently,

$$
\Pi_{k}\left(g \cdot \mathcal{X}_{\mathfrak{t}}(k)\right)=\eta(g) \cdot \Pi_{k}\left(\mathcal{X}_{\mathfrak{t}}(k)\right) \subset h \cdot \mathcal{X}_{\mathfrak{s}^{\prime}}(k)=\mathcal{X}_{\mathfrak{s}^{\prime}}(k) .
$$

Since $\operatorname{rk}(\mathfrak{g} / \mathfrak{n})=\operatorname{rk}(\mathfrak{g})-\operatorname{dim}_{k} \mathfrak{n},[11,(1.6)]$ and (1) imply that $\left.\Pi_{k}\right|_{\mathcal{X}_{\mathfrak{t}}(k)}: \mathcal{X}_{\mathfrak{t}}(k) \longrightarrow$ $\mathcal{X}_{\mathfrak{s}^{\prime}}(k)$ and $\left.\Pi_{k}\right|_{g \cdot \mathcal{X}_{\mathfrak{t}}(k)}: g \cdot \mathcal{X}_{\mathfrak{t}}(k) \longrightarrow \mathcal{X}_{\mathfrak{s}^{\prime}}(k)$ are dominant morphisms of irreducible varieties. Accordingly,

$$
\Pi_{k}\left(\mathcal{X}_{\mathfrak{t}}(k)\right) \cap \Pi_{k}\left(g \cdot \mathcal{X}_{\mathfrak{t}}(k)\right) \neq \emptyset,
$$

and there exist $\varphi_{1}, \varphi_{2} \in \mathcal{X}_{\mathfrak{t}}(k)$ such that

$$
\left.\pi \circ \varphi_{1} \circ g^{-1}\right|_{\mathfrak{s}^{\prime}}=\left.\pi \circ \varphi\right|_{\mathfrak{s}^{\prime}} .
$$

Consequently,

$$
\varphi_{1} \circ g^{-1}(s)=\varphi_{2}(s)+\psi(s) \quad \forall s \in \mathfrak{s}^{\prime}
$$

for some $\psi \in \operatorname{Lie}_{p}\left(\mathfrak{s}^{\prime}, \mathfrak{n}\right)(k)$. According to (5.11) we have $\left.\varphi_{1}\right|_{\mathfrak{n}}=\mathrm{id}_{\mathfrak{n}}=\left.\varphi_{2}\right|_{\mathfrak{n}}$. Thus, the homomorphism $\omega \in \operatorname{Lie}_{p}(\mathfrak{t}, \mathfrak{t})(k)$ given by

$$
\omega(s+n):=g^{-1}(s)-\psi(s)+n \quad \forall s \in \mathfrak{s}^{\prime}, n \in \mathfrak{n}
$$

satisfies

$$
\varphi_{1} \circ \omega=\varphi_{2} .
$$

In particular, $\omega$ is injective, so that $\omega \in \operatorname{Nor}_{G_{\mathfrak{t}}(k)}(\mathfrak{n})$. Since $\omega^{-1} \cdot \mathcal{X}_{\mathfrak{t}}(k) \cap \mathcal{X}_{\mathfrak{t}}(k) \neq \emptyset$, we conclude that $\omega \in S(\mathfrak{g}, \mathfrak{t})$. Directly from the definitions we obtain

$$
\eta(\omega)=\left.\operatorname{pr}_{\mathfrak{s}^{\prime}} \circ \omega\right|_{\mathfrak{s}^{\prime}}=\left.\operatorname{pr}_{\mathfrak{s}^{\prime}} \circ g^{-1}\right|_{\mathfrak{s}^{\prime}}=h^{-1},
$$

whence $\eta\left(\omega^{-1}\right)=h$.

(3) According to (5.11), we have $S(\mathfrak{g}, \mathfrak{t}) \subset \operatorname{Cent}_{G_{\mathfrak{t}}(k)}(\mathfrak{n})$. The assertion now follows from the inclusion $\eta(S(\mathfrak{g}, \mathfrak{t})) \subset S\left(\mathfrak{g} / \mathfrak{n}, \mathfrak{s}^{\prime}\right)$. 
(4) Since $\mathcal{Y}^{\mathfrak{n}}(k) \subset \mathcal{T}_{\mathfrak{g}}(k)$ is open, (5.4) and (1) imply that $\Pi_{k}$ is a smooth morphism with finite fibres, which, owing to [6, $(\mathrm{I}, \S 4,4.7)]$ is thereby étale. By virtue of Lemma 1.3 the morphism

$$
\Psi: \mathcal{Y}^{\mathfrak{n}}(k) \longrightarrow \hat{\mathcal{T}}_{\mathfrak{g} / \mathfrak{n}}(k) ; \varphi \mapsto\left[\Pi_{k}(\varphi)\right]
$$

is, as a composite of étale morphisms, étale. In view of the equivariance of $\Pi_{k}, \Psi$ is constant on the $\operatorname{Nor}_{G_{\mathfrak{t}}(k)}(\mathfrak{n})$-orbits of $\mathcal{Y}^{\mathfrak{n}}(k)$ and thus induces a morphism

$$
\hat{\Pi}_{k}: \mathcal{Y}^{\mathfrak{n}}(k) / \operatorname{Nor}_{G_{\mathfrak{t}}(k)}(\mathfrak{n}) \longrightarrow \hat{\mathcal{T}}_{\mathfrak{g} / \mathfrak{n}}(k) ;[\varphi] \mapsto\left[\Pi_{k}(\varphi)\right],
$$

whose composite with the étale and faithfully flat morphism $\mathcal{Y}^{\mathfrak{n}}(k) \longrightarrow \mathcal{Y}^{\mathfrak{n}}(k) /$ $\operatorname{Nor}_{G_{\mathfrak{t}}(k)}(\mathfrak{n})$ is just $\Psi$. Thanks, to Lemma 1.4 the morphism $\hat{\Pi}_{k}$ is étale. By part (1) $\hat{\Pi}_{k}$ is bijective, and [18, (3.6.3.4)] shows that $\hat{\Pi}_{k}$ is an isomorphism.

Consider the morphism

$$
\Lambda: \mathcal{Y}^{\mathfrak{n}}(k) \longrightarrow \hat{\mathcal{T}}_{\mathfrak{g}}(k) ; \varphi \mapsto[\varphi] .
$$

Since $\mathcal{Y}^{\mathfrak{n}}(k) \subset \mathcal{T}_{\mathfrak{g}}(k)$ is a union of connected components, Lemma 1.3 shows that $\Lambda$ is étale. As before, we see that the induced morphism

$$
\tilde{\Lambda}: \mathcal{Y}^{\mathfrak{n}}(k) / \operatorname{Nor}_{G_{\mathfrak{t}}(k)}(\mathfrak{n}) \longrightarrow \hat{\mathcal{T}}_{\mathfrak{g}}(k)
$$

enjoys the same property.

Let $[\psi]$ be an element $\hat{\mathcal{T}}_{\mathfrak{g}}(k)$. In view of $\operatorname{dim}_{k} \psi^{-1}(\mathfrak{n})=\operatorname{dim}_{k} \mathfrak{n}$, there exists $g \in G_{\mathfrak{t}}(k)$ with $(g \cdot \psi)^{-1}(\mathfrak{n})=\mathfrak{n}$. Thus, $g \cdot \psi \in \mathcal{Y}^{\mathfrak{n}}(k)$, and $\Lambda(g \cdot \psi)=[\psi]$. Consequently, $\tilde{\Lambda}$ is surjective.

If $\varphi, \varphi^{\prime}$ belong to $\mathcal{Y}^{\mathfrak{n}}(k)$ such that $\tilde{\Lambda}([\varphi])=\tilde{\Lambda}\left(\left[\varphi^{\prime}\right]\right)$, then there exists $g \in G_{\mathfrak{t}}(k)$ with $\varphi^{\prime}=g \cdot \varphi$. Hence $\mathfrak{n}=(g \cdot \varphi)^{-1}(\mathfrak{n})=g(\mathfrak{n})$, so that $g \in \operatorname{Nor}_{G_{\mathfrak{t}}(k)}(\mathfrak{n})$. Consequently, $[\varphi]=\left[\varphi^{\prime}\right]$, and $\tilde{\Lambda}$ is injective.

As before, the bijective, étale morphism $\tilde{\Lambda}$ is an isomorphism. Thus, $\hat{\Pi}_{k} \circ \tilde{\Lambda}^{-1}$ is the desired isomorphism.

To verify the last assertion we consider the commutative diagram

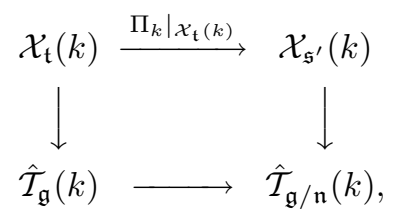

whose vertical arrows are surjective and whose bottom horizontal arrow is bijective. It follows that for any $\psi \in \mathcal{X}_{\mathfrak{s}^{\prime}}(k)$ there exist $h \in S\left(\mathfrak{g}^{\prime}, \mathfrak{s}^{\prime}\right)$ and $\varphi \in \mathcal{X}_{\mathfrak{t}}(k)$ such that

$$
\psi=h \cdot \Pi_{k}(\varphi) .
$$

In view of (2) we may write $h=\eta(g)$ for some $g \in S(\mathfrak{g}, \mathfrak{t})$, whence

$$
\psi=\Pi_{k}(g \cdot \varphi) \in \Pi_{k}\left(\mathcal{X}_{\mathfrak{t}}(k)\right) .
$$

In view of (1), (5.4) and [6, $(\mathrm{I}, \S 4,4.7)]\left.\Pi_{k}\right|_{\mathcal{X}_{\mathbf{t}}(k)}$ is étale and thus induces a bijective, étale morphism $\tilde{\Pi}_{k}: \mathcal{X}_{\mathfrak{t}}(k) /(\operatorname{ker} \eta \cap S(\mathfrak{g}, \mathfrak{t})) \longrightarrow \mathcal{X}_{\mathfrak{s}^{\prime}}(k)$, which, by [18, (3.6.3.4)], is an isomorphism.

Remark. The example following (4.1) shows that $(5.12(4))$ does not hold for arbitrary tori of $\mathfrak{g}$. 


\section{The StRUCture of $\mathfrak{g}$ AND $\mathcal{T}_{\mathfrak{g}}$}

In this section we study the interplay between the structure of a restricted Lie algebra $(\mathfrak{g},[p])$ and that of its varieties of tori. We have already observed that nilpotent Lie algebras are characterized by their varieties being finite.

6.1. Solvable Lie algebras. We apply the results of $\S 5$ to study the scheme of (embeddings of) tori of maximal dimension in case the underlying finite-dimensional Lie algebra $\mathfrak{g}$ is solvable. By way of illustration, we first consider the case when $\mathfrak{g}=\operatorname{Lie}(\mathcal{G})$ is the Lie algebra of a connected, smooth, solvable group $\mathcal{G}$. According to general theory [28, (6.11)] the group $\mathcal{G}$ decomposes into a semidirect product

$$
\mathcal{G}=\mathcal{U} \rtimes \mathcal{T}
$$

with a normal, unipotent $\operatorname{subgroup} \mathcal{U}$ and a maximal diagonalizable subgroup $\mathcal{T}$. Since $\mathfrak{g}=\operatorname{Lie}(\mathcal{U}) \oplus \operatorname{Lie}(\mathcal{T})$, the $p$-subalgebra $\mathfrak{t}:=\operatorname{Lie}(\mathcal{T})$ is a maximal torus of $\mathfrak{g}$. Theorem 2.1 now provides an isomorphism $\mathcal{X}_{\mathfrak{t}}(k) \cong \mathcal{U}(k) / \operatorname{Cen}_{\mathcal{U}(k)}(\mathfrak{t})$. The general structure theory of smooth unipotent algebraic groups entails that the latter space is isomorphic to the $\left(\operatorname{dim}_{k} \mathfrak{g}-\operatorname{rk}(\mathfrak{g})\right)$-dimensional affine space (cf. 6. (IV, §4, 3.4, $3.16)])$.

For any natural number $n$, we let $c_{n}$ be the order of the group $\mathrm{GL}_{n}\left(\mathbb{F}_{p}\right)$. Recall that $\mathfrak{g}$ is referred to as trigonalizable if every simple restricted $\mathfrak{g}$-module is onedimensional. We say that $\mathfrak{g}$ is supersolvable if $\mathfrak{g}$ possesses a filtration by $p$-ideals with one-dimensional factors. Trigonalizable restricted Lie algebras are supersolvable.

Theorem 6.1. Let $(\mathfrak{g},[p])$ be trigonalizable of dimension $\ell, \mathfrak{t} \subset \mathfrak{g}$ a torus of dimension $\mu(\mathfrak{g})$. Then the following statements hold:

(1) $\mathcal{T}_{\mathfrak{g}}(k)$ is the disjoint union of $c_{\mu(\mathfrak{g})}$ copies of $\mathbb{A}^{\ell-\mathrm{rk}(\mathfrak{g})}$.

(2) $\quad \hat{\mathcal{T}}_{\mathfrak{g}}(k) \cong \mathbb{A}^{\ell-\mathrm{rk}(\mathfrak{g})}$.

Proof. (1) By general theory (cf. proof of [29, (V.8.6)]), the Lie algebra $\mathfrak{g}$ decomposes into a semidirect sum $\mathfrak{g}=\mathfrak{t} \oplus \mathfrak{m}$ with a $p$-nilpotent ideal $\mathfrak{m}$. We proceed by induction on $\operatorname{dim}_{k} \mathfrak{m}$, with the case $\operatorname{dim}_{k} \mathfrak{m}=0$ following from the isomorphism $G_{\mathfrak{t}}(k) \cong \mathrm{GL}_{\mu(\mathfrak{g})}\left(\mathbb{F}_{p}\right)$. Assuming $\operatorname{dim}_{k} \mathfrak{m}>0$, we let $(0) \neq \mathfrak{n} \subset \mathfrak{m}$ be a strongly abelian $p$-ideal of $\mathfrak{g}$. Then $\mathfrak{g}^{\prime}:=\mathfrak{g} / \mathfrak{n} \cong \mathfrak{t} \oplus(\mathfrak{m} / \mathfrak{n})$ is trigonalizable, and the inductive hypothesis implies that the connected component $\mathcal{X}_{\mathfrak{t}}^{\prime}(k)$ of $\mathcal{T}_{\mathfrak{g}^{\prime}}(k)$ containing the standard embedding is an affine space $\mathbb{A}^{r}$.

Owing to Proposition 5.7 the morphism $\left.\Pi_{k}\right|_{\Pi_{k}^{-1}\left(\mathcal{X}_{\mathfrak{t}}^{\prime}(k)\right)}: \Pi_{k}^{-1}\left(\mathcal{X}_{\mathfrak{t}}^{\prime}(k)\right) \longrightarrow \mathcal{X}_{\mathfrak{t}}^{\prime}(k)$ is an algebraic vector bundle. It thus corresponds to a finitely generated projective module $P$ over $k\left[\mathcal{X}_{\mathfrak{t}}^{\prime}(k)\right] \cong k\left[X_{1}, \ldots, X_{r}\right]$ (cf. [9, p. 623]). Thanks to the QuillenSuslin theorem (cf. [24, Thm.4]) the module $P$ is free. Accordingly, the above bundle is trivial, whence $\Pi_{k}^{-1}\left(\mathcal{X}_{\mathfrak{t}}^{\prime}(k)\right) \cong \mathcal{X}_{\mathfrak{t}}^{\prime}(k) \times \mathbb{A}^{n} \cong \mathbb{A}^{r+n}$ for some $n \in \mathbb{N}$. In particular, $\Pi_{k}^{-1}\left(\mathcal{X}_{\mathfrak{t}}^{\prime}(k)\right)$ is an irreducible variety containing the component $\mathcal{X}_{\mathfrak{t}}(k)$. This implies $\mathcal{X}_{\mathfrak{t}}(k) \cong \mathbb{A}^{r+n}$, where $r+n=\ell-\operatorname{rk}(\mathfrak{g})$ for dimension reasons (cf. (3.5), [11, (1.6)]).

In view of (4.1) and the $G_{\mathrm{t}}(k)$-equivariance of $\Pi_{k}$, we see that the inverse image of each connected component of $\mathcal{T}_{\mathfrak{g}^{\prime}}(k)$ is $\mathcal{T}_{\mathfrak{g}}(k)$ is a connected component of $\mathcal{T}_{\mathfrak{g}}(k)$. Consequently, the number of these components is the same for $\mathcal{T}_{\mathfrak{g}}(k)$ and $\mathcal{T}_{\mathfrak{g}^{\prime}}(k)$, as desired.

(2) Owing to (4.1) $\left[G_{\mathfrak{t}}(k): S(\mathfrak{g}, \mathfrak{t})\right]$ is the number of components of the variety $\mathcal{T}_{\mathfrak{g}}(k)$. In view of $(1) S(\mathfrak{g}, \mathfrak{t})=\{1\}$ is the trivial subgroup, so that our assertion follows directly from (1) and $4.1(3))$. 
Remark. The triviality of the toral stabilizer $S(\mathfrak{g}, \mathfrak{t})$ corresponds to the fact that the Weyl group $W(\mathcal{G}, T)$ of a connected, solvable, smooth $k$-group is trivial (cf. [15] (19.4)]).

Theorem 6.2. Let $(\mathfrak{g},[p])$ be a solvable, restricted Lie algebra of dimension $\ell, \mathfrak{t} \subset \mathfrak{g}$ a torus of dimension $\mu(\mathfrak{g})$. Then the following statements hold:

(1) $S(\mathfrak{g}, \mathfrak{t})$ is a p-group.

(2) If $p \geq 3$, then $\hat{\mathcal{T}}_{\mathfrak{g}}(k) \cong \mathbb{A}^{\ell-\mathrm{rk}(\mathfrak{g})}$.

(3) If $\mathfrak{g}$ is supersolvable, then $\hat{\mathcal{T}}_{\mathfrak{g}}(k) \cong \mathbb{A}^{\ell-\mathrm{rk}(\mathfrak{g})}$, and $S(\mathfrak{g}, \mathfrak{t})$ is an elementary abelian p-group.

Proof. (1) We proceed by induction on $\ell$, the case $\ell=1$ being trivial. Let $\mathfrak{n} \subset \mathfrak{g}$ be a minimal $p$-ideal. As $\mathfrak{g}$ is solvable, $\mathfrak{n}$ is abelian, so that $\mathfrak{n}^{[p]} \subset C(\mathfrak{g})$. Consequently, $\mathfrak{n}$ is either strongly abelian or toral.

If $\mathfrak{n}$ is strongly abelian, then (5.6) yields $S(\mathfrak{g}, \mathfrak{t}) \cong S(\mathfrak{g} / \mathfrak{n}, \mathfrak{t})$, and the inductive hypothesis implies the assertion.

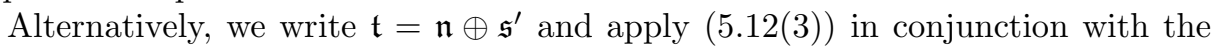
inductive hypothesis.

(2) As in (1) we proceed by induction on $\ell$ and let $\mathfrak{n} \triangleleft \mathfrak{g}$ be a minimal $p$-ideal. If $\mathfrak{n}$ is toral, then the inductive step follows from (5.12(4)). Alternatively, (5.10) in conjunction with the inductive hypothesis provides an algebraic vector bundle

$$
\hat{\mathcal{T}}_{\mathfrak{g}}(k) \longrightarrow \mathbb{A}^{q},
$$

which, by the arguments of the proof of Theorem 6.1 is trivial. Consequently, we have $\hat{\mathcal{T}}_{\mathfrak{g}}(k) \cong \mathbb{A}^{r}$, with $r=\ell-\operatorname{rk}(\mathfrak{g})$ for dimension reasons.

(3) Let $T(\mathfrak{g})$ be the largest toral ideal of $\mathfrak{g}$. As $\mathfrak{g}$ is supersolvable, the factor algebra $\mathfrak{g}^{\prime}:=\mathfrak{g} / T(\mathfrak{g})$ is trigonalizable (cf. [10, (2.3)]). By combining Theorem 6.1] with $(5.12(4))$, we obtain $\hat{\mathcal{T}}_{\mathfrak{g}}(k) \cong \hat{\mathcal{T}}_{\mathfrak{g}^{\prime}}(k) \cong \mathbb{A}^{\ell-\operatorname{rk}(\mathfrak{g})}$.

In view of (6.1) the stabilizer of the standard component of $\mathcal{T}_{\mathfrak{g}^{\prime}}(k)$ is trivial. Thus, $(\mathbf{5 . 1 2}(3))$ implies that $S(\mathfrak{g}, \mathfrak{t}) \hookrightarrow \operatorname{Lie}_{p}\left(\mathfrak{s}^{\prime}, \mathfrak{n}\right)(k)$ is elementary abelian.

Examples. (1) Consider the three-dimensional Lie algebra $\mathfrak{g}:=k t \oplus k x \oplus k z$ with product and $p$-map given by

$$
[t, x]=x,[t, z]=0=[x, z] ; t^{[p]}=t, x^{[p]}=z=z^{[p]} .
$$

Then $\mathfrak{t}:=k t \oplus k z$ is a torus of maximal dimension, and direct computation shows that $k z=C(\mathfrak{g})$ is the only one-dimensional $p$-ideal of the solvable Lie algebra $\mathfrak{g}$. Consequently, $\mathfrak{g}$ is not trigonalizable, and thus cannot be the Lie algebra of a smooth solvable group. If $p \geq 3$, it follows that $\mathfrak{g}$ is not the Lie algebra of any smooth group (cf. [14, (12.1)]). We have seen at the beginning of $\S 4$ that the connected component $\mathcal{X}_{k t}(k)$ of the Lie algebra $\mathfrak{g}^{\prime}:=\mathfrak{g} / C(\mathfrak{g})$ consists of those elements $\varphi \in \mathcal{T}_{\mathfrak{g}^{\prime}}(k)$ such that $\varphi(t)=t+\alpha x$ for some $\alpha \in k$. According to (6.1) the variety $\mathcal{T}_{\mathfrak{g}^{\prime}}(k)$ has $p-1$ irreducible components, each of which is isomorphic to $\mathbb{A}^{1}$.

Now let $\varphi$ be an element of $\mathcal{X}_{\mathfrak{t}}(k)$. By (5.11) we have $\varphi(z)=z$, and since $\Pi_{k}(\varphi) \in \mathcal{X}_{k t}(k)$, the above computations imply $\varphi(t)=t+\alpha x+\beta z$, with $\alpha, \beta \in k$ satisfying $\beta^{p}-\beta+\alpha^{p}=0$. We call this map $\varphi_{(\alpha, \beta)}$.

The variety $V:=\left\{(\alpha, \beta) \in \mathbb{A}^{2} ; \beta^{p}-\beta+\alpha^{p}=0\right\}$ is isomorphic to $\mathbb{A}^{1}$. In particular, $V$ is irreducible, and by the above, the image of morphism

$$
V \longrightarrow \mathcal{T}_{\mathfrak{g}}(k) ;(\alpha, \beta) \mapsto \varphi_{(\alpha, \beta)}
$$


contains the connected component $\mathcal{X}_{\mathfrak{t}}(k)$. Hence the image equals $\mathcal{X}_{\mathfrak{t}}(k)$ and we have an isomorphism $V \cong \mathcal{X}_{\mathfrak{t}}(k)$, sending $(0,0)$ onto the standard embedding $\iota$.

Let $g$ be an element of $S(\mathfrak{g}, \mathfrak{t})$. Then we have $g(z)=z$ as well as $g \in \mathcal{X}_{\mathfrak{t}}(k)$. Accordingly, $g(t)=t+\gamma z$ with $\gamma \in \mathbb{F}_{p}$. Conversely, we have $g \cdot \iota \in \mathcal{X}_{\mathfrak{t}}(k)$ for every such $g$, so that $S(\mathfrak{g}, \mathfrak{t}) \cong \mathbb{Z} /(p)$ and $\mathcal{T}_{\mathfrak{g}}(k)$ possesses $\frac{c_{2}}{p}=\left(p^{2}-1\right)(p-1)$ irreducible components.

(2) Let $\mathfrak{h}:=k x \oplus k y \oplus k z$ be the three-dimensional Heisenberg algebra, whose structure is given by

$$
[x, y]=z,[x, z]=0=[y, z] ; x^{[p]}=0=y^{[p]}, z^{[p]}=z .
$$

This algebra possesses a $p$-dimensional simple restricted module $V$, on which $z$ operates via the identity. We consider the semidirect product $\mathfrak{g}:=\mathfrak{h} \ltimes V$, where $V$ is strongly abelian and note that $\mathfrak{g}$ is not supersolvable. By the proof of (6.1) the variety $\mathcal{T}_{\mathfrak{g}}(k)$ given by the maximal torus $k z$ is the disjoint union of $p-1$ copies of $\mathbb{A}^{p}$. In view of (5.6) we have $S(\mathfrak{g}, k z)=S(\mathfrak{h}, k z)=\{1\}$, so that $\hat{\mathcal{T}}_{\mathfrak{g}}(k) \cong \mathcal{X}_{k z}(k) \cong \mathbb{A}^{p}$.

6.2. Lie algebras whose varieties of tori are $\mathbb{A}^{n}$. Let $(\mathfrak{g},[p])$ be a finitedimensional restricted Lie algebra, $\mathfrak{t} \subset \mathfrak{g}$ a torus of maximal dimension. Aside from $\mathfrak{s l}(2)$ the examples of the varieties of tori $\mathcal{X}_{\mathfrak{t}}(k)$ and $\hat{\mathcal{T}}_{\mathfrak{g}}(k)$ computed so far are full affine spaces. In this section we investigate this situation more systematically.

By choosing a basis of $\mathfrak{t}$ consisting of toral elements, we identify the automorphism group $G_{\mathfrak{t}}(k)$ with the general linear group $\operatorname{GL}(\mu(\mathfrak{g}))\left(\mathbb{F}_{p}\right)$ over the Galois field. Given a finite group $G$, we denote by $\operatorname{Ab}(G):=G /(G, G)$ its abelianization.

Theorem 6.3. Let $(\mathfrak{g},[p])$ be an $\ell$-dimensional restricted Lie algebra such that $\hat{\mathcal{T}}_{\mathfrak{g}}(k) \cong \mathbb{A}^{\ell-\operatorname{rk}(\mathfrak{g})}$. Then $\operatorname{Ab}(S(\mathfrak{g}, \mathfrak{t}))$ is a p-group, and $S(\mathfrak{g}, \mathfrak{t}) \subset \operatorname{SL}(\mu(\mathfrak{g}))\left(\mathbb{F}_{p}\right)$.

Proof. We put $n:=\ell-\operatorname{rk}(\mathfrak{g})$. Let $\mathcal{F}$ be an algebraic $k$-group. Following [6] (III, $\S 4,4.1)]$ we denote by $\tilde{H}^{1}\left(\mathbb{A}^{n}, \mathcal{F}\right)$ the set of isomorphism classes of principal fibre bundles (torseurs) over $\mathbb{A}^{n}$ with structure group $\mathcal{F}$ (respectively). In the following, we let $\mu_{k}=\operatorname{Spec}_{k}\left(k[T]_{T}\right)$ be the multiplicative group. For each $m \in \mathbb{N}$ the group scheme $\mu_{m}:=\operatorname{Spec}\left(k[T] /\left(T^{m}-1\right)\right)$ is the kernel of the morphism $\mu_{k} \longrightarrow \mu_{k} ; r \mapsto$ $r^{m}$.

Let $q$ be a prime power not divisible by $p, N \triangleleft S(\mathfrak{g}, \mathfrak{t})$ a normal subgroup such that $S(\mathfrak{g}, \mathfrak{t}) / N \cong \mathbb{Z} /(q)$. We consider the variety $Y:=\mathcal{X}_{\mathfrak{t}}(k) / N$. Owing to [6. (III, $\S 2,6.1)]$ the morphism $Y \longrightarrow \hat{\mathcal{T}}_{\mathfrak{g}}(k)$ is a principal fibre bundle with structure group $\mathbb{Z} /(q) \cong \mu_{q}(k)$.

According to [․, (III, $\S 4,5.5)]$ we have an exact sequence

$$
k\left[X_{1}, \ldots, X_{n}\right]^{*} / k\left[X_{1}, \ldots, X_{n}\right]^{* q} \longrightarrow \tilde{H}^{1}\left(\mathbb{A}^{n}, \mu_{q}\right) \longrightarrow \operatorname{Pic}\left(\mathbb{A}^{n}\right)_{q} .
$$

Here the left-hand group is the factor group of invertible elements by its subgroup of $q$-th powers, Pic $\left(\mathbb{A}^{n}\right)$ denotes the Picard group of $\mathbb{A}^{n}$ (cf. [6. (III, §4, 4.4)]) and $\operatorname{Pic}\left(\mathbb{A}^{n}\right)_{q}:=\left\{x \in \operatorname{Pic}\left(\mathbb{A}^{n}\right) ; x^{q}=1\right\}$. In view of (cf. [20, (20.7)]) and the surjectivity of $k^{*} \longrightarrow k^{*} ; \alpha \mapsto \alpha^{q}$ the extreme terms of the sequence are trivial, whence $\tilde{H}^{1}\left(\mathbb{A}^{n}, \mu_{q}\right)=\{1\}$. As a result, the principal fibre bundle $Y \longrightarrow \hat{\mathcal{T}}_{\mathfrak{g}}(k)$ is trivial, so that $Y \cong \mathbb{Z} /(q) \times \hat{\mathcal{T}}_{\mathfrak{g}}(k)$. Since $Y$ is irreducible, we have $q=1$, whence $N=S(\mathfrak{g}, \mathfrak{t})$.

We now decompose $\mathrm{Ab}(S(\mathfrak{g}, \mathfrak{t}))$ into a direct product of cyclic subgroups of prime power order. By the above, only factors of order a power of $p$ occur, so that $\operatorname{Ab}(S(\mathfrak{g}, \mathfrak{t}))$ is a $p$-group. 
The restriction of the homomorphism det $: G_{\mathfrak{t}}(k) \longrightarrow \mathbb{F}_{p}^{\times}$to $S(\mathfrak{g}, \mathfrak{t})$ factors through to a homomorphism $\mathrm{Ab}(S(\mathfrak{g}, \mathfrak{t})) \longrightarrow \mathbb{F}_{p}^{\times}$. By the first part of the proof this homomorphism is trivial, so that $S(\mathfrak{g}, \mathfrak{t}) \subset \mathrm{SL}(\mu(\mathfrak{g}))\left(\mathbb{F}_{p}\right)$.

Corollary 6.4. Let $(\mathfrak{g},[p])$ be an $\ell$-dimensional restricted Lie algebra with $\mu(\mathfrak{g})=1$, $\mathfrak{t} \subset \mathfrak{g}$ is a one-dimensional torus.

(1) If $\hat{\mathcal{T}}_{\mathfrak{g}}(k) \cong \mathbb{A}^{\ell-\mathrm{rk}(\mathfrak{g})}$, then $S(\mathfrak{g}, \mathfrak{t})=\{1\}$, and $\mathcal{X}_{\mathfrak{t}}(k) \cong \mathbb{A}^{\ell-\mathrm{rk}(\mathfrak{g})}$.

(2) If $\mathfrak{g}$ is solvable, then $S(\mathfrak{g}, \mathfrak{t})=\{1\}$, and $\mathcal{X}_{\mathfrak{t}}(k) \cong \mathbb{A}^{\ell-\mathrm{rk}(\mathfrak{g})} \cong \hat{\mathcal{T}}_{\mathfrak{g}}(k)$.

Proof. (1) By assumption $S(\mathfrak{g}, \mathfrak{t}) \subset \mathbb{F}_{p}^{\times}$is an abelian group, which, in view of (6.3), is also a $p$-group. Consequently, $S(\mathfrak{g}, \mathfrak{t})=\{1\}$, and (4.1) yields $\mathcal{X}_{\mathfrak{t}}(k) \cong \hat{\mathcal{T}}_{\mathfrak{g}}(k) \cong$ $\mathbb{A}^{\ell-\operatorname{rk}(\mathfrak{g})}$

(2) This is a combination of Theorem 6.2, whose proof in this situation also works for $p=2$ (cf. (5.10), and (1).

By combining (4.1) and (6.4) we obtain that the variety of embeddings of a solvable Lie algebra $\mathfrak{g}$ of multiplicative $\operatorname{rank} \mu(\mathfrak{g})=1$ is the disjoint union of $p-1$ copies of some $\mathbb{A}^{n}$.

The following result provides a partial converse to Theorem [6.2.

Theorem 6.5. Suppose that $p \geq 3$. Let $\mathfrak{g}=\operatorname{Lie}(\mathcal{G})$ be the Lie algebra of an $\ell$ dimensional smooth group $\mathcal{G}, \mathfrak{t} \subset \mathfrak{g}$ a maximal torus. If $\hat{\mathcal{T}}_{\mathfrak{g}}(k) \cong \mathbb{A}^{\ell-\mathrm{rk}(\mathfrak{g})}$, then $\mathcal{G}$ is solvable.

Proof. Owing to [14, (13.3)] there exists a maximal torus $T \subset \mathcal{G}$ such that $\mathfrak{t}=$ $\operatorname{Lie}(T)$. Moreover, (4.4) implies $\operatorname{dim}_{k} \mathfrak{t}=\mu(\mathfrak{g})$.

We consider the homomorphism

$$
f: W(\mathcal{G}, T) \longrightarrow S(\mathfrak{g}, \mathfrak{t}) ;\left.[g] \mapsto \operatorname{Ad}(g)\right|_{\mathfrak{t}}
$$

Let $R$ be the set of roots of $\mathcal{G}$ relative to $T$ (in the sense of [28, (9.1.4)]). The group $\operatorname{Hom}\left(k^{\times}, T\right)$ of cocharacters of $T$ will be denoted $Y(T)$. Given $\alpha \in R$ we let $\alpha^{\vee} \in Y(T)$ be the corresponding coroot. For each $\lambda \in Y(T)$ the element $h_{\lambda}:=d(\lambda)(1) \in \mathfrak{t}$ satisfies $h_{\lambda}^{[p]}=h_{\lambda}$, and $\mathfrak{t}$ is generated by $\left\{h_{\lambda} ; \lambda \in Y(T)\right\}$. As usual, we put $h_{\alpha}:=h_{\alpha}$ for every $\alpha \in R$.

Let $\alpha \in R$ be a root, $s_{\alpha} \in W(\mathcal{G}, T)$ the corresponding reflection. Then $s_{\alpha}$ acts on $Y(T)$ via

$$
s_{\alpha}(\lambda)=\lambda-\langle\alpha, \lambda\rangle \alpha^{\vee} \quad \forall \lambda \in Y(T) .
$$

If $n_{\alpha} \in \mathcal{G}(k)$ represents $s_{\alpha}$, and $i_{n_{\alpha}}$ denotes the inner automorphism of $\mathcal{G}(k)$ effected by $n_{\alpha}$, then we have

$$
\begin{aligned}
f\left(s_{\alpha}\right)\left(h_{\lambda}\right) & =\operatorname{Ad}\left(n_{\alpha}\right)\left(h_{\lambda}\right)=d\left(i_{n_{\alpha}}\right)(d(\lambda)(1))=d\left(i_{n_{\alpha}} \circ \lambda\right)(1)=d\left(\lambda-\langle\alpha, \lambda\rangle \alpha^{\vee}\right)(1) \\
& =h_{\lambda}-\langle\alpha, \lambda\rangle h_{\alpha} .
\end{aligned}
$$

Direct computation shows $\langle\alpha, \lambda\rangle \cdot 1=d(\alpha)\left(h_{\lambda}\right)$, so that

$$
f\left(s_{\alpha}\right)(t)=t-d(\alpha)(t) h_{\alpha} \quad \forall t \in \mathfrak{t} .
$$

Since $d(\alpha)\left(h_{\alpha}\right)=\left\langle\alpha, \alpha^{\vee}\right\rangle=2$ and $p \geq 3$, we can thus find a toral basis of $\mathfrak{t}$ such that $f\left(s_{\alpha}\right) \notin \mathrm{SL}(\mu(\mathfrak{g}))\left(\mathbb{F}_{p}\right)$. As this contradicts Theorem 6.3 we conclude that $R=\emptyset$. A consecutive application of [28, (9.1.2)] and [28, (7.3.5)] yields the solvability of $\mathcal{G}(k)$. The assertion now follows from [6, (IV, $\S 4,2.4)]$. 
Remark. (1) The example SL(2) shows that (6.5) does not hold at even characteristic.

(2) Let $\mathfrak{t} \subset \mathfrak{s l}(2)$ be the standard torus. By (6.5) the variety $\hat{\mathcal{T}}_{\mathfrak{s l}(2)}(k)$, which we computed in $\S 4$, is not isomorphic to $\mathbb{A}^{2}$ whenever $p \geq 3$.

(3) Suppose that $p \geq 3$, and let $\mathcal{G}$ be a smooth group with maximal torus $T \subset \mathcal{G}$. In view of (4.7) and (6.5) the assumption $\mathcal{G}(k) / \operatorname{Nor}_{\mathcal{G}(k)}(T) \cong \mathbb{A}^{\operatorname{dim} \mathcal{G}-\operatorname{rk}(\mathfrak{g})}$ implies the solvability of $\mathcal{G}$.

Corollary 6.6. Suppose that $p \geq 5$, and let $(\mathfrak{g},[p])$ be an $\ell$-dimensional restricted simple Lie algebra of classical or Cartan type. If $\mathfrak{t} \subset \mathfrak{g}$ is a torus of maximal dimension, then $\hat{\mathcal{T}}_{\mathfrak{g}}(k) \neq \mathbb{A}^{\ell-\mathrm{rk}(\mathfrak{g})}$.

Proof. If $\mathfrak{g}$ is classical, then (4.6) shows that $S(\mathfrak{g}, \mathfrak{t})$ is generated by reflections, so that $S(\mathfrak{g}, \mathfrak{t}) \not \subset \operatorname{SL}(\mu(\mathfrak{g}))\left(\mathbb{F}_{p}\right)$. Thanks to $(6.3)$ this yields $\hat{\mathcal{T}}_{\mathfrak{g}}(k) \neq \mathbb{A}^{\ell-\operatorname{rk}(\mathfrak{g})}$.

Alternatively, $\mathfrak{g}$ is of Cartan type, and by Demuškin's work [7, 8] all maximal tori have the same dimension. Accordingly, [29, (IV.2.5, IV.3.6, IV.4.6, IV.5.7)] shows that we can choose $\mathfrak{t}$ in such a way that there exists a $p$-subalgebra $\mathfrak{q} \subset \mathfrak{g}$ containing $\mathfrak{t}$, which is a direct sum of a classical Lie algebra $\mathfrak{h}$ and a torus $\mathfrak{n}$ of dimension $\leq 1$. (In case of the Witt algebra $W(1 ; 1)$, we have $\mathfrak{q}=\mathfrak{s l}(2)$.) Consequently, $S(\mathfrak{q}, \mathfrak{t}) \subset S(\mathfrak{g}, \mathfrak{t})$. Choose a torus $\mathfrak{s}^{\prime} \subset \mathfrak{h}$ with $\mathfrak{t}=\mathfrak{s}^{\prime} \oplus \mathfrak{n}$. There results a morphism

$$
\mathcal{T}_{\mathfrak{h}}(k) \longrightarrow \mathcal{T}_{\mathfrak{q}}(k) ; \varphi \mapsto \varphi \oplus \operatorname{id}_{\mathfrak{n}},
$$

which gives rise to an embedding

$$
\lambda: S\left(\mathfrak{h}, \mathfrak{s}^{\prime}\right) \longrightarrow S(\mathfrak{q}, \mathfrak{t}) \quad ; \quad g \mapsto g \oplus \mathrm{id}_{\mathfrak{n}} .
$$

Since $\operatorname{det}(\lambda(g))=\operatorname{det}(g)$ for every $g \in S\left(\mathfrak{h}, \mathfrak{s}^{\prime}\right)$, the first part of the proof yields $S(\mathfrak{q}, \mathfrak{t}) \not \subset \mathrm{SL}(\mu(\mathfrak{g}))\left(\mathbb{F}_{p}\right)$. Consequently, $S(\mathfrak{g}, \mathfrak{t}) \not \subset \mathrm{SL}(\mu(\mathfrak{g}))\left(\mathbb{F}_{p}\right)$, and (6.3) implies the desired result.

We conclude this section by considering restricted Lie algebras whose varieties of embeddings are disjoint unions of full affine spaces. The following result shows, in particular, that the irreducible components of $\mathcal{T}_{\mathfrak{s l}(2)}(k)$ are not isomorphic to $\mathbb{A}^{2}$.

Theorem 6.7. Let $(\mathfrak{g},[p])$ be an $\ell$-dimensional restricted Lie algebra such that $\mathcal{X}_{\mathfrak{t}}(k) \cong \mathbb{A}^{\ell-\mathrm{rk}(\mathfrak{g})}$. Then the following statements hold:

(1) $S(\mathfrak{g}, \mathfrak{t})$ is a p-group.

(2) If $\mathfrak{g}=\operatorname{Lie}(\mathcal{G})$ is the Lie algebra of a smooth group $\mathcal{G}$ and $p \geq 3$, then $\mathcal{G}$ is solvable.

(3) If $p \geq 5$, then $\mathfrak{g}$ is not simple of classical or Cartan type.

Proof. (1) Let $q \neq p$ be a prime and suppose that $H \subset S(\mathfrak{g}, \mathfrak{t})$ is a $q$-subgroup. According to [6. $(\mathrm{IV}, \S 4,4.3)]$ there exists a fixed-point $x \in \mathcal{X}_{\mathfrak{t}}(k)^{H}$. As $S(\mathfrak{g}, \mathfrak{t})$ operates freely on $\mathcal{X}_{\mathfrak{t}}(k)$, it follows that $H=\{1\}$. Thus, by Sylow's Theorem, $q$ does not divide the order of $S(\mathfrak{g}, \mathfrak{t})$, and $S(\mathfrak{g}, \mathfrak{t})$ is a $p$-group.

(2) This is a direct consequence of (1) and the remark following (4.7).

(3) Let $\mathfrak{g}$ be simple of classical or Cartan type. According to the proof of (6.6) the group $S(\mathfrak{g}, \mathfrak{t})$ is not a $p$-group.

Remark. According to the Block-Wilson classification theorem [3] every restricted simple Lie algebra $\mathfrak{g}$ of characteristic $p>7$ is either classical or of Cartan type. 
Corollary 6.8. Let $\mathcal{G}$ be an $\ell$-dimensional smooth algebraic $k$-group of characteristic $p \geq 3, \mathfrak{t} \subset \operatorname{Lie}(\mathcal{G})$ a maximal torus. Then the following statements are equivalent:

(1) $\hat{\mathcal{T}}_{\mathrm{Lie}(\mathcal{G})}(k) \cong \mathbb{A}^{\ell-\operatorname{rk}(\operatorname{Lie}(\mathcal{G}))}$.

(2) $\mathcal{G}$ is solvable.

(3) $\mathcal{X}_{\mathfrak{t}}(k) \cong \mathbb{A}^{\ell-\operatorname{rk}(\operatorname{Lie}(\mathcal{G}))}$.

Proof. (1) $\Rightarrow(2)$ See 6.5 .

$(2) \Rightarrow(3)$ By the Lie-Kolchin Theorem the Lie algebra Lie $(\mathcal{G})$ of the smooth solvable group $\mathcal{G}$ is trigonalizable. Hence ([6.I) implies $\mathcal{X}_{\mathfrak{t}}(k) \cong \mathbb{A}^{\ell-\operatorname{rk}(\operatorname{Lie}(\mathcal{G}))}$.

$(3) \Rightarrow(1)$ See (6.7) and (6.1).

Example. We show by an example that Corollary 6.8 does not hold for arbitrary supersolvable restricted Lie algebras. Consider the "diamond algebra" $\mathfrak{g}=k t \oplus$ $k x \oplus k y \oplus k z$, whose bracket and $p$-map are given by

$$
[t, x]=x,[t, y]=-y,[t, z]=0,[x, y]=z,[x, z]=0=[y, z]
$$

and

$$
t^{[p]}=t, x^{[p]}=0=y^{[p]}, z^{[p]}=z,
$$

respectively.

The algebra $\mathfrak{g}$ is supersolvable, and $\mathfrak{n}:=k z$ is a toral ideal of $\mathfrak{g}$. Since $\mathfrak{t}:=k t \oplus k z$ is a self-centralizing torus, (3.5) and (3.6) imply $\mu(\mathfrak{g})=\operatorname{rk}(\mathfrak{g})=2$. Consequently, (6.2) yields $\hat{\mathcal{T}}_{\mathfrak{g}}(k) \cong \mathbb{A}^{2}$.

The factor algebra $\mathfrak{g}^{\prime}:=\mathfrak{g} / \mathfrak{n}$ is trigonalizable of $\operatorname{rank} \operatorname{rk}\left(\mathfrak{g}^{\prime}\right)=1$ and with maximal torus $\mathfrak{s}^{\prime}:=k t$. In view of (6.1) $\mathcal{T}_{\mathfrak{g}^{\prime}}(k)$ is the disjoint union of $p-1$ copies of $\mathbb{A}^{2}$. In fact, every $\varphi \in \mathcal{T}_{\mathfrak{g}^{\prime}}(k)$ is given by

$$
\varphi(t)=\lambda(\varphi) t+\alpha(\varphi) x+\beta(\varphi) y
$$

with $\lambda(\varphi) \in \mathbb{F}_{p}^{\times}, \alpha(\varphi), \beta(\varphi) \in k$. Accordingly, the elements of $\mathcal{X}_{\mathfrak{s}^{\prime}}^{\prime}(k)$ are of the form

$$
\varphi(t)=t+\alpha(\varphi) x+\beta(\varphi) y .
$$

Now let $\psi$ be an element of $\mathcal{X}_{\mathfrak{t}}(k)$. Then (5.11) and (5.12(4)) imply

$$
\psi(t)=t+\alpha(\psi) x+\beta(\psi) y+\gamma(\psi) z \quad ; \quad \psi(z)=z .
$$

For $p \geq 3$, the $p$-map on the Heisenberg algebra $\mathfrak{h}:=k x \oplus k y \oplus k z$ is $p$-semilinear, so that Jacobson's formula for the $p$-map yields

$$
\begin{aligned}
\psi(t)^{[p]} & =(t+\alpha(\psi) x+\beta(\psi) y)^{[p]}+\gamma(\psi)^{p} z \\
& =t+\alpha(\psi) x+\beta(\psi) y-\alpha(\psi) \beta(\psi) z+\gamma(\psi)^{p} z .
\end{aligned}
$$

This formula also holds for $p=2$. As a result, we obtain

$$
\gamma(\psi)^{p}-\gamma(\psi)-\alpha(\psi) \beta(\psi)=0
$$

We consider the irreducible variety

$$
\mathcal{V}:=\left\{(\alpha, \beta, \gamma) \in k^{3} ; \gamma^{p}-\gamma-\alpha \beta=0\right\} .
$$

The foregoing observations provide a morphism

$$
\mathcal{V} \longrightarrow \mathcal{T}_{\mathfrak{g}}(k),
$$

whose image contains the irreducible component $\mathcal{X}_{\mathfrak{t}}(k)$. Accordingly, the above map induces an isomorphism

$$
\mathcal{V} \stackrel{\sim}{\longrightarrow} \mathcal{X}_{\mathfrak{t}}(k) .
$$


It now follows that $S(\mathfrak{g}, \mathfrak{t})=\mathcal{X}_{\mathfrak{t}}(k) \cap G_{\mathfrak{t}}(k) \cong \mathbb{Z} /(p)$ (see remark (2) preceding (4.1)).

We continue by showing that $\mathcal{V}$ is not a full affine space. To that end we consider its coordinate ring

$$
A:=k[X, Y, Z] /\left(Z^{p}-Z-X Y\right) .
$$

Thus, $A$ is an integral domain, which is generated by the residue classes $x, y, z$ of $X, Y, Z$. Using the fact that the canonical projection restricts to an embedding $k[X, Z] \hookrightarrow A$ as well as $x^{m(f)} f \in k[x, y]$ for all $f \in A$ one shows that $x \in A$ is irreducible. Applying the automorphism that interchanges $x$ and $y$ and fixes $z$ one sees that $y$ is also irreducible. The identity

$$
x y=z\left(z^{p-1}-1\right)=z(z-1)\left(1+z+\cdots+z^{p-2}\right)
$$

now shows that $A$ is not factorial (this also holds for $p=2$ ). Hence $A$ is not isomorphic to a polynomial ring, and (6.8) does not hold for $\mathfrak{g}$.

\section{VARIETIES OF $p$-SUBALGeBRAS}

In the following sections we are going to employ schemes of tori to study subvarieties of the Grassmannian $\mathrm{Gr}_{d}(\mathfrak{g})(k)$ of $d$-dimensional subspaces of a given finitedimensional restricted Lie algebra $(\mathfrak{g},[p])$. Let $\operatorname{Sub}_{d}(\mathfrak{g})(k), \operatorname{Solv}_{d}(\mathfrak{g})(k), \operatorname{Nil}_{d}(\mathfrak{g})(k)$, and $\operatorname{Ab}_{d}(\mathfrak{g})(k)$ be the subsets of $\operatorname{Gr}_{d}(\mathfrak{g})(k)$ consisting of all $d$-dimensional $p$ subalgebras, all $d$-dimensional solvable $p$-subalgebras, all $d$-dimensional nilpotent $p$-subalgebras, and all $d$-dimensional abelian $p$-subalgebras of $\mathfrak{g}$, respectively. A $p$-subalgebra $\mathfrak{h} \subset \mathfrak{g}$ is said to act triangulably on $\mathfrak{g}$ if every composition factor of the $\mathfrak{h}$-module $\mathfrak{g}$ is one-dimensional. We let $\operatorname{Trig}_{d}(\mathfrak{g})(k)$ be the set of those $d$-dimensional $p$-subalgebras of $\mathfrak{g}$ that act triangulably on $\mathfrak{g}$. As a consequence of the following results, the five sets defined above are projective varieties.

Let $\operatorname{Gr}_{d}(\mathfrak{g}): M_{k} \longrightarrow$ Ens be the Grassmann scheme, i.e., the $k$-functor that assigns to every commutative $k$-algebra $R$ the set $\operatorname{Gr}_{d}(\mathfrak{g})(R)$ of $R$-direct summands of $\mathfrak{g} \otimes_{k} R$ of rank $d$ (cf. [16, (I.1.9)]). We begin by considering the subfunctor $\operatorname{Sub}_{d}(\mathfrak{g}) \subset \operatorname{Gr}_{d}(\mathfrak{g})$ that is given by

$$
\operatorname{Sub}_{d}(\mathfrak{g})(R):=\left\{M \in \operatorname{Gr}_{d}(\mathfrak{g})(R) ;[M, M] \subset M, M^{[p]} \subset M\right\}
$$

for every commutative $k$-algebra $R \in M_{k}$. Recall that the $R$-Lie algebra $\mathfrak{g} \otimes_{k} R$ carries the structure of a restricted Lie algebra via

$$
(x \otimes r)^{[p]}:=x^{[p]} \otimes r^{p} \quad \forall x \in \mathfrak{g}, r \in R .
$$

Proposition 7.1. Let $(\mathfrak{g},[p])$ be a finite-dimensional restricted Lie algebra. Then $\operatorname{Sub}_{d}(\mathfrak{g})$ is a closed subfunctor of $\operatorname{Gr}_{d}(\mathfrak{g})$.

Proof. Let $\varphi: R \longrightarrow S$ be a homomorphism of $k$-algebras. Since $\left(\mathfrak{g} \otimes_{k} R\right) \otimes_{R} S \longrightarrow$ $\mathfrak{g} \otimes_{k} S ; x \otimes r \otimes s \mapsto x \otimes \varphi(r) s$ is an isomorphism of restricted $k$-Lie algebras, it follows that $\operatorname{Gr}_{d}(\mathfrak{g})(\varphi)$ sends $\operatorname{Sub}_{d}(\mathfrak{g})(R)$ to $\operatorname{Sub}_{d}(\mathfrak{g})(S)$. Consequently, $\operatorname{Sub}_{d}(\mathfrak{g})$ is a subfunctor of the $k$-functor $\mathrm{Gr}_{d}(\mathfrak{g})$.

To verify the closedness of $\operatorname{Sub}_{d}(\mathfrak{g})$, we show that for every commutative $k$ algebra $A$ and every morphism $f: \operatorname{Spec}_{k}(A) \rightarrow \operatorname{Gr}_{d}(\mathfrak{g})$, the subfunctor $f^{-1}\left(\operatorname{Sub}_{d}(\mathfrak{g})\right)$ $\subset \operatorname{Spec}_{k}(A)$ is closed (cf. [16, (I.1.12)]). By Yoneda's Lemma [16, (I.1.3)] the morphism $f$ corresponds to an $A$-point $M \in \mathrm{Gr}_{d}(\mathfrak{g})(A)$. 
For a basis $\left\{v_{1}, \ldots, v_{n}\right\}$ of $\mathfrak{g}$ let $\alpha_{i j \ell} \in k$ be the structure constants that are given by

$$
\left[v_{i}, v_{j}\right]=\sum_{\ell=1}^{n} \alpha_{i j \ell} v_{\ell}, \quad 1 \leq i, j \leq n .
$$

We denote by $\left\{m_{1}, \ldots, m_{d}\right\}$ a set of generators of the locally free $A$-module $M$ of rank $d$, and define elements $a_{r i}, b_{r i} \in A$ via

$$
m_{r}=\sum_{i=1}^{n} v_{i} \otimes a_{r i}, \quad 1 \leq r \leq d,
$$

and

$$
m_{r}^{[p]}=\sum_{i=1}^{n} v_{i} \otimes b_{r i}, \quad 1 \leq r \leq d,
$$

respectively. By definition of $\operatorname{Gr}_{d}(\mathfrak{g})(A)$, there exists an $A$-submodule $N \subset \mathfrak{g} \otimes_{k} A$ such that

$$
\mathfrak{g} \otimes_{k} A=M \oplus N
$$

and we denote by pr: $\mathfrak{g} \otimes_{k} A \longrightarrow N \hookrightarrow \mathfrak{g} \otimes_{k} A$ the corresponding projection. This $A$-linear map is given by

$$
\operatorname{pr}\left(v_{j} \otimes 1\right)=\sum_{i=1}^{n} v_{i} \otimes c_{i j}, \quad 1 \leq j \leq n .
$$

We let $I \subset A$ be the ideal generated by the elements

$h_{r s t}:=\sum_{\ell=1}^{n} \sum_{i=1}^{n} \sum_{j=1}^{n} \alpha_{i j \ell} a_{r i} a_{s j} c_{t \ell} \quad ; \quad g_{t r}:=\sum_{\ell=1}^{n} c_{t \ell} b_{r \ell}, \quad 1 \leq t \leq n, 1 \leq r, s \leq d$.

Now let $\varphi: A \longrightarrow R$ be a homomorphism of $k$-algebras. Then we have

$$
\mathfrak{g} \otimes_{k} R=\left(M_{R}\right) \oplus\left(N_{R}\right),
$$

where $X_{R}=X \otimes_{A} R$ for $X \in\{M, N\}$. The corresponding projection $\operatorname{pr}_{R}: \mathfrak{g} \otimes_{k}$ $R \longrightarrow N_{R} \hookrightarrow \mathfrak{g} \otimes_{k} R$ is given by

$$
\operatorname{pr}_{R}\left(v_{j} \otimes 1\right)=\sum_{i=1}^{n} v_{i} \otimes \varphi\left(c_{i j}\right), \quad 1 \leq j \leq n .
$$

In view of

$$
\left[m_{r} \otimes 1, m_{s} \otimes 1\right]=\sum_{\ell=1}^{n} v_{\ell} \otimes\left(\sum_{i=1}^{n} \sum_{j=1}^{n} \alpha_{i j \ell} \varphi\left(a_{r i}\right) \varphi\left(a_{s j}\right)\right)
$$

we have

$$
\operatorname{pr}_{R}\left(\left[m_{r} \otimes 1, m_{s} \otimes 1\right]\right)=\sum_{t=1}^{n} v_{t} \otimes \varphi\left(h_{r s t}\right) .
$$

Similarly, we obtain

$$
\operatorname{pr}_{R}\left(\left(m_{r} \otimes 1\right)^{[p]}\right)=\sum_{t=1}^{n} v_{t} \otimes \varphi\left(g_{t r}\right)
$$

Now suppose that $\varphi(I)=(0)$. Then the foregoing identities imply $\left[M_{R}, M_{R}\right] \subset$ ker $\operatorname{pr}_{R}=M_{R}$ as well as $\left(m_{r} \otimes 1\right)^{[p]} \in M_{R}$ for $r \in\{1, \ldots, d\}$. By general properties of the $p$-map (cf. [29, (II, §1)]), this entails $M_{R}^{[p]} \subset M_{R}$. Consequently, $M_{R}=\operatorname{Gr}_{d}(\mathfrak{g})(\varphi)(M)$ belongs to $\operatorname{Sub}_{d}(\mathfrak{g})(R)$. The same arguments show that 
$M_{R} \in \operatorname{Sub}_{d}(\mathfrak{g})(R)$ implies $I \subset \operatorname{ker} \varphi$. Consequently, $f^{-1}\left(\operatorname{Sub}_{d}(\mathfrak{g})\right)$ is a closed subfunctor of $\operatorname{Spec}_{k}(A)$, as desired.

Remark. Consider the $k$-functor $\operatorname{Id}_{d}(\mathfrak{g}): M_{k} \longrightarrow$ Ens given by

$$
\operatorname{Id}_{d}(\mathfrak{g})(R):=\left\{M \in \operatorname{Sub}_{d}(\mathfrak{g})(R) ;\left[\mathfrak{g} \otimes_{k} R, M\right] \subset M\right\}
$$

for every commutative $k$-algebra $R$. As in (7.1) one readily sees that $\operatorname{Id}_{d}(\mathfrak{g})$ is a closed subfunctor of $\operatorname{Sub}_{d}(\mathfrak{g})$ and $\operatorname{Gr}_{d}(\mathfrak{g})$.

Recall that the nilpotency class of a nilpotent Lie algebra $\mathfrak{h}$ is the smallest number $c$ such that the $(c+1)$ st term $\mathfrak{h}^{(c+1)}$ of the lower central series of $\mathfrak{h}$ vanishes. Given $c \geq 1$, we consider the subfunctor $\operatorname{Nil}_{d}^{c}(\mathfrak{g}) \subset \operatorname{Sub}_{d}(\mathfrak{g})$ of $p$-subalgebras of nilpotency class $\leq c$. Note that $\operatorname{Nil}_{d}^{1}(\mathfrak{g})=: \operatorname{Ab}_{d}(\mathfrak{g})$ coincides with the subfunctor of abelian $p$-subalgebras of $\mathfrak{g}$. The proof of the following result, being analogous to that of (7.1), will be omitted.

Proposition 7.2. $\operatorname{Nil}_{d}^{c}(\mathfrak{g})$ is a closed subfunctor of $\operatorname{Sub}_{d}(\mathfrak{g})$ for every $c \geq 1$.

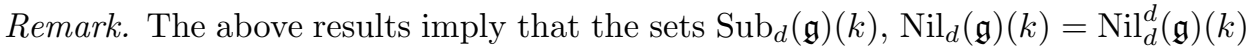
and $\mathrm{Ab}_{d}(\mathfrak{g})(k)$ of rational points of these functors are closed subsets of the Grassmann variety. The following argument shows that triangulably acting $p$-subalgebras also enjoy this property.

Setting $n:=\operatorname{dim}_{k} \mathfrak{g}$, we note that a $p$-subalgebra $\mathfrak{h} \subset \mathfrak{g}$ belongs to $\operatorname{Trig}_{d}(\mathfrak{g})(k)$ if and only if $x^{[p]^{n}} \in C(\mathfrak{g})$ for every $x \in[\mathfrak{h}, \mathfrak{h}]$. Consider the morphism

$$
f: \mathfrak{g}^{2 d} \longrightarrow \mathfrak{g} ; \quad\left(x_{1}, \ldots, x_{d}, y_{1}, \ldots, y_{d}\right) \mapsto\left(\sum_{i=1}^{d}\left[x_{i}, y_{i}\right]\right)^{[p]^{n}} .
$$

Since $\Sigma_{m}:=\left\{\left(V, x_{1}, \ldots, x_{m}\right) \in \operatorname{Gr}_{d}(\mathfrak{g})(k) \times \mathfrak{g}^{m} ;\left(x_{1}, \ldots, x_{m}\right) \in V^{m}\right\}$ is a closed subset of the variety $\operatorname{Gr}_{d}(\mathfrak{g})(k) \times \mathfrak{g}^{m}$ for any $m \geq 1$, the set $Z:=\{(V, z) \in$ $\left.\Sigma_{2 d} ; f(z) \in C(\mathfrak{g})\right\}$ is closed in $\operatorname{Gr}_{d}(\mathfrak{g})(k) \times \mathfrak{g}^{2 d}$. The fibres of the projection $\pi: Z \longrightarrow \operatorname{Gr}_{d}(\mathfrak{g})(k)$ are given by

$$
\pi^{-1}(\pi(V, v)) \cong\left\{z \in V^{2 d} ; f(z) \in C(\mathfrak{g})\right\} .
$$

Upper semicontinuity of fibre dimension then implies that the set

$$
L:=\left\{(V, v) \in Z ; f\left(V^{2 d}\right) \subset C(\mathfrak{g})\right\}
$$

is closed. By considering the inverse image of $L$ under the morphism $\operatorname{Gr}_{d}(\mathfrak{g})(k) \longrightarrow$ $Z ; V \mapsto(V, 0)$, we see that $K:=\left\{\mathfrak{h} \in \operatorname{Sub}_{d}(\mathfrak{g})(k) ; f\left(\mathfrak{h}^{2 d}\right) \subset C(\mathfrak{g})\right\}$ also enjoys this property. Since $[\mathfrak{h}, \mathfrak{h}]=\left\{\sum_{i=1}^{d}\left[x_{i}, y_{i}\right] ; x_{i}, y_{i} \in \mathfrak{h}\right\}$ for every $\mathfrak{h} \in \operatorname{Sub}_{d}(\mathfrak{g})(k)$, we have $K=\operatorname{Trig}_{d}(\mathfrak{g})(k)$.

Similar arguments show that $\operatorname{Solv}_{d}(\mathfrak{g})(k)$ is a closed subset of $\operatorname{Sub}_{d}(\mathfrak{g})(k)$.

In the sequel we want to define open subfunctors of $\operatorname{Nil}_{d}^{c}(\mathfrak{g})$. To that end we require subsidiary results on subvarieties of the Grassmannian.

Let $Y \subset \mathfrak{g}$ and $X \subset \operatorname{Gr}_{d}(\mathfrak{g})(k)$ be closed subsets. We consider the closed subvariety

$$
X^{Y}:=\{(y, V) \in Y \times X ; y \in V\}
$$

of $Y \times X$. The projections of $Y \times X$ onto the two factors induce morphisms $\pi_{Y}: X^{Y} \longrightarrow Y$ and $\pi_{X}: X^{Y} \longrightarrow X$, respectively. Since the projective variety $\operatorname{Gr}_{d}(\mathfrak{g})$ is complete (cf. [21, (I, $\left.\left.\left.\S 9\right)\right]\right), \pi_{Y}$ is a closed map. 
Lemma 7.3. Let $Y \subset \mathfrak{g}$ be a closed, conical variety. Then the map

$$
\operatorname{Gr}_{d}(\mathfrak{g})(k) \longrightarrow \mathbb{N}_{0} \quad ; \quad V \mapsto \operatorname{dim} V \cap Y
$$

is upper semicontinuous.

Proof. Since $\pi_{Y}$ induces isomorphisms

$$
\pi_{\mathrm{Gr}_{d}(\mathfrak{g})(k)}^{-1}\left(\pi_{\mathrm{Gr}_{d}(\mathfrak{g})(k)}(y, V)\right) \cong V \cap Y
$$

sending $(y, V)$ to $y$, (1.1) yields the upper semicontinuity of the map

$$
\operatorname{Gr}_{d}(\mathfrak{g})(k)^{Y} \longrightarrow \mathbb{N}_{0} \quad ; \quad(y, V) \mapsto \operatorname{dim}_{y} V \cap Y .
$$

As every irreducible component of the closed, conical variety $V \cap Y$ contains the origin, we obtain the assertion by considering the inverse images under the morphism $\operatorname{Gr}_{d}(\mathfrak{g})(k) \longrightarrow \operatorname{Gr}_{d}(\mathfrak{g})(k)^{Y} ; V \mapsto(0, V)$.

We next consider the sets $\operatorname{SNor}_{d}(\mathfrak{g})(k), \operatorname{Car}_{d}(\mathfrak{g})(k)$, and $\operatorname{Tor}_{d}(\mathfrak{g})(k)$ of $d$-dimensional self-normalizing $p$-subalgebras, of $d$-dimensional Cartan subalgebras, and of $d$-dimensional tori of $\mathfrak{g}$, respectively.

Lemma 7.4. Let $(\mathfrak{g},[p])$ be a finite-dimensional restricted Lie algebra.

(1) $\operatorname{SNor}_{d}(\mathfrak{g})(k)$ is an open subset of $\operatorname{Sub}_{d}(\mathfrak{g})(k)$.

(2) $\operatorname{Car}_{d}(\mathfrak{g})(k)$ is an open subset of $\mathrm{Nil}_{d}(\mathfrak{g})(k)$.

(3) $\operatorname{Tor}_{d}(\mathfrak{g})(k)$ is an open subset of $\mathrm{Ab}_{d}(\mathfrak{g})(k)$.

Proof. We consider the morphism

$$
\psi: \operatorname{End}_{k}(\mathfrak{g}) \times \operatorname{Gr}_{d}(\mathfrak{g})(k) \times \mathfrak{g} \longrightarrow \operatorname{Gr}_{d}(\mathfrak{g})(k) \times \mathfrak{g} ; \quad(f, V, x) \mapsto(V, f(x)),
$$

and recall that $\Sigma_{1}=\left\{(V, x) \in \mathrm{Gr}_{d}(\mathfrak{g})(k) \times \mathfrak{g} ; x \in V\right\}$ is a closed subvariety of $\operatorname{Gr}_{d}(\mathfrak{g}) \times \mathfrak{g}$. Thus, $\Omega(\psi):=\left(\operatorname{End}_{k}(\mathfrak{g}) \times \Sigma_{1}\right) \cap \psi^{-1}\left(\Sigma_{1}\right)=\left\{(f, V, x) ; x \in V \cap f^{-1}(V)\right\}$ is a closed subset of $\operatorname{End}_{k}(\mathfrak{g}) \times \operatorname{Gr}_{d}(\mathfrak{g})(k) \times \mathfrak{g}$. We denote by $\gamma: \Omega(\psi) \longrightarrow \operatorname{End}_{k}(\mathfrak{g}) \times$ $\mathrm{Gr}_{d}(\mathfrak{g})(k)$ the restriction of the projection onto the first two factors. Then we have $\gamma^{-1}(\gamma(f, V, x)) \cong V \cap f^{-1}(V)$, so that $\varepsilon_{\gamma}(f, V, x) \leq d$. Upper semicontinuity of fibre dimension implies that the set

$$
C:=\left\{(f, V, x) \in \Omega(\psi) ; \varepsilon_{\gamma}(f, V, x)=d\right\}=\{(f, V, x) \in \Omega(\psi) ; f(V) \subset V\}
$$

is closed in $\Omega(\psi)$. By considering the morphism

$$
\omega: \operatorname{End}_{k}(\mathfrak{g}) \times \operatorname{Gr}_{d}(\mathfrak{g})(k) \longrightarrow \Omega(\psi) ;(f, V) \mapsto(f, V, 0),
$$

we conclude that

$$
D:=\omega^{-1}(C)=\left\{(f, V) \in \operatorname{End}_{k}(\mathfrak{g}) \times \operatorname{Gr}_{d}(\mathfrak{g})(k) ; f(V) \subset V\right\}
$$

is closed. Since the adjoint representation ad $: \mathfrak{g} \longrightarrow \operatorname{End}_{k}(\mathfrak{g})$ induces a morphism

$$
\zeta: \mathfrak{g} \times \operatorname{Gr}_{d}(\mathfrak{g})(k) \longrightarrow \operatorname{End}_{k}(\mathfrak{g}) \times \operatorname{Gr}_{d}(\mathfrak{g})(k) ;(x, V) \mapsto(\operatorname{ad} x, V),
$$

the set

$$
E:=\zeta^{-1}\left(D \cap\left(\operatorname{End}_{k}(\mathfrak{g}) \times \operatorname{Sub}_{d}(\mathfrak{g})(k)\right)\right)=\left\{(x, \mathfrak{h}) \in \mathfrak{g} \times \operatorname{Sub}_{d}(\mathfrak{g})(k) ;[x, \mathfrak{h}] \subset \mathfrak{h}\right\}
$$

is a closed subvariety of $\mathfrak{g} \times \operatorname{Sub}_{d}(\mathfrak{g})(k)$. Let $\pi: E \longrightarrow \operatorname{Sub}_{d}(\mathfrak{g})(k)$ be the restriction of the projection $\mathfrak{g} \times \operatorname{Sub}_{d}(\mathfrak{g})(k) \longrightarrow \operatorname{Sub}_{d}(\mathfrak{g})(k)$. Then we have $\pi^{-1}(\pi(x, \mathfrak{h})) \cong$ $\operatorname{Nor}_{\mathfrak{g}}(\mathfrak{h})$, the normalizer of $\mathfrak{h}$ in $\mathfrak{g}$. Consequently,

$$
\varepsilon_{\pi}(x, \mathfrak{h})=\operatorname{dim}_{k} \operatorname{Nor}_{\mathfrak{g}}(\mathfrak{h}) \quad \text { for every }(x, \mathfrak{h}) \in E,
$$


so that $d \leq \varepsilon_{\pi}(x, \mathfrak{h}) \leq \operatorname{dim}_{k} \mathfrak{g}$. Observe that $\operatorname{SNor}_{d}(\mathfrak{g})(k)$ and $\operatorname{Id}_{d}(\mathfrak{g})(k)$ are the inverse images of the sets $\left\{(x, \mathfrak{h}) \in E ; \operatorname{dim} \varepsilon_{\pi}(x, \mathfrak{h})=d\right\}$ and $\{(x, \mathfrak{h}) \in$ $\left.E ; \operatorname{dim} \varepsilon_{\pi}(x, \mathfrak{h})=\operatorname{dim}_{k} \mathfrak{g}\right\}$ under the morphism $\mathfrak{h} \mapsto(0, \mathfrak{h})$, respectively. Thus, the assertions (1) and (2) now follow from (1.1.

(3) Since $\mathcal{V}_{\mathfrak{g}}=\left\{x \in \mathfrak{g} ; x^{[p]}=0\right\}$ is conical and $\mathcal{V}_{\mathfrak{h}}=\mathfrak{h} \cap \mathcal{V}_{\mathfrak{g}}$ for every $\mathfrak{h} \in$ $\operatorname{Sub}_{d}(\mathfrak{g})(k)$, Lemma 7.3 implies that

$$
U:=\left\{\mathfrak{h} \in \operatorname{Sub}_{d}(\mathfrak{g})(k) ; \operatorname{dim} \mathcal{V}_{\mathfrak{h}}=0\right\}
$$

is open in $\operatorname{Sub}_{d}(\mathfrak{g})(k)$. As $\mathcal{V}_{\mathfrak{h}}$ is conical, we have $\mathcal{V}_{\mathfrak{h}}=\{0\}$ for every $\mathfrak{h} \in U$. In view of [29 (3.6), (3.10)] the set $U$ therefore coincides with $\operatorname{Tor}_{d}(\mathfrak{g})(k)$.

For a torus $\mathfrak{t} \subset \mathfrak{g}$ of dimension $d$, we consider the subfunctors $\operatorname{Tor}_{d}(\mathfrak{g})$ and $\operatorname{Car}_{d}(\mathfrak{g})$ of $\operatorname{Sub}_{d}(\mathfrak{g})$ that are given by

$$
\operatorname{Tor}_{d}(\mathfrak{g})(R):=\left\{\mathfrak{m} \in \operatorname{Ab}_{d}(\mathfrak{g})(R) ; \mathfrak{m}(x) \text { is a torus } \forall x \in \operatorname{Spec}_{k}(R)(k)\right\}
$$

as well as

$\operatorname{Car}_{d}(\mathfrak{g})(R):=\left\{\mathfrak{m} \in \operatorname{Nil}_{d}^{d}(\mathfrak{g})(R) ; \mathfrak{m}(x) \subset \mathfrak{g}\right.$ is a Cartan subalgebra $\left.\forall x \in \operatorname{Spec}_{k}(R)(k)\right\}$. In view of [16, (I.1.9(2), I.1.12(6))] the following proposition shows that these functors are subschemes of $\mathrm{Gr}_{d}(\mathfrak{g})$.

Proposition 7.5. The following statements hold:

(1) $\operatorname{Tor}_{d}(\mathfrak{g})$ is an open subfunctor of $\mathrm{Ab}_{d}(\mathfrak{g})$.

(2) $\operatorname{Car}_{d}(\mathfrak{g})$ is an open subfunctor of $\operatorname{Nil}_{d}^{d}(\mathfrak{g})$.

Proof. It is clear that $\operatorname{Tor}_{d}(\mathfrak{g})$ and $\operatorname{Car}_{d}(\mathfrak{g})$ are subfunctors of $\operatorname{Ab}_{d}(\mathfrak{g})$ and $\operatorname{Nil}_{d}^{d}(\mathfrak{g})$, respectively.

(1) By definition the set of $k$-rational points of $\operatorname{Tor}_{d}(\mathfrak{g})$ coincides with the $\operatorname{Tor}_{d}(\mathfrak{g})(k)$ which we have defined before. Thanks to Lemma $7.4(3) \operatorname{Tor}_{d}(\mathfrak{g})(k) \subset$ $\mathrm{Ab}_{d}(\mathfrak{g})(k)$ is open, and we may now apply [6. $\left.(\mathrm{I}, \S 3,6.8)\right]$ to obtain our assertion.

(2) This follows as in (1) by employing $(7.4(2))$.

Proposition 7.6. Let $(\mathfrak{g},[p])$ be a finite-dimensional restricted Lie algebra. Then the following statements hold:

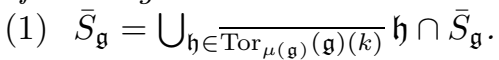

(2) Every Cartan subalgebra of $\mathfrak{g}$ contains an abelian p-subalgebra of dimension $\mu(\mathfrak{g})$.

Proof. (1) Setting $X:=\overline{\operatorname{Tor}_{\mu(\mathfrak{g})}(\mathfrak{g})(k)}$ we consider the variety $X^{\bar{S}_{\mathfrak{g}}}$ along with the projection $\pi_{\bar{S}_{\mathfrak{g}}}: X^{\bar{S}_{\mathfrak{g}}} \longrightarrow \bar{S}_{\mathfrak{g}}$. Since the map $\pi_{\bar{S}_{\mathfrak{g}}}$ is closed, we see that $\operatorname{im} \pi_{\bar{S}_{\mathfrak{g}}}=$ $\bigcup_{\mathfrak{h} \in X} \mathfrak{h} \cap \bar{S}_{\mathfrak{g}}$ is a closed subset of $\bar{S}_{\mathfrak{g}}$.

We let $\mathfrak{t} \subset \mathfrak{g}$ be a torus of dimension $\mu(\mathfrak{g})$ and consider the morphism

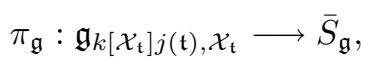

whose image is given by $\operatorname{im} \pi_{\mathfrak{g}}=\bigcup_{\varphi \in \mathcal{X}_{\mathfrak{t}}(k)} \varphi(\mathfrak{t})$. We put $Y:=\overline{\operatorname{im} \pi_{\mathfrak{g}}}$, and apply

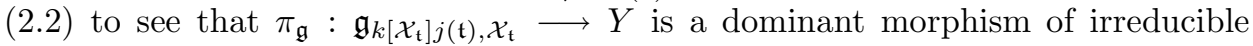
varieties. Thanks to $(3.5(1))$ im $\pi_{\mathfrak{g}}$ meets the open set $\operatorname{Regs}(\mathfrak{g})$ of $S_{\mathfrak{g}}$. Since $C_{\mathfrak{g}}(t)$ is nilpotent for every $t \in \operatorname{Regs}(\mathfrak{g})$, Proposition 2.3 ensures the finiteness of the fibres $\pi_{\mathfrak{g}}^{-1}(t), t \in \operatorname{Regs}(\mathfrak{g}) \cap \operatorname{im} \pi_{\mathfrak{g}}$. Accordingly, the generic fibre of $\pi_{\mathfrak{g}}$ has dimension

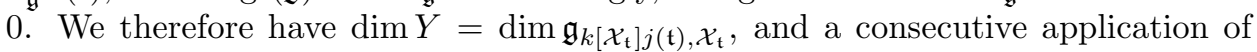
(3.5)(1)) and (2.2) yields $\operatorname{dim} Y=\operatorname{dim}_{k} \mathfrak{g}-\operatorname{rk}(\mathfrak{g})+\mu(\mathfrak{g})$. 
It now follows from (3.7) that im $\pi_{\bar{S}_{\mathfrak{g}}}$ contains the dense subset $\bigcup_{\mathfrak{t} \in \operatorname{Tor}_{\mu(\mathfrak{g})}(\mathfrak{g})(k)} \mathfrak{t}$ of $\bar{S}_{\mathfrak{g}}$. Consequently, (1) holds.

(2) Let $\mathfrak{h} \subset \mathfrak{g}$ be a Cartan subalgebra. In view of 3.2 we can find a semisimple element $s \in S_{\mathfrak{g}}$ such that $\mathfrak{h}=C_{\mathfrak{g}}(s)$. According to (1), the element $s$ is contained in an abelian $p$-subalgebra $\mathfrak{s} \subset \mathfrak{g}$ of dimension $\mu(\mathfrak{g})$. Thus, $\mathfrak{s} \subset C_{\mathfrak{g}}(s)=\mathfrak{h}$, so that assertion (2) follows.

Since a self-centralizing torus is a Cartan subalgebra, (7.6) immediately implies that such a torus has maximal dimension (cf. (3.6)).

Let $(\mathfrak{g},[p])$ be a finite-dimensional restricted Lie algebra, $\mathfrak{t} \subset \mathfrak{g}$ a torus with centralizer $C_{\mathfrak{g}}(\mathfrak{t})$ of dimension $d$. According to our observations of Section 2, the map

$$
\zeta_{\mathfrak{t}}: \mathcal{X}_{\mathfrak{t}}(k) \longrightarrow \operatorname{Gr}_{d}(\mathfrak{g})(k) ; x \mapsto \tilde{\mathfrak{g}}_{0}(x)
$$

is well defined, and its image contains the $p$-subalgebra $C_{\mathfrak{g}}(\mathfrak{t})$. We denote by $\Omega_{\mathfrak{t}}$ the closure of $\operatorname{im} \zeta_{\mathfrak{t}}$ in $\operatorname{Gr}_{d}(\mathfrak{g})(k)$. Part (2) of the following result generalizes [30. $(3.11)]$.

Proposition 7.7. Let $\mathfrak{t} \subset \mathfrak{g}$ be a torus. Then the following statements hold:

(1) The map $\zeta_{\mathfrak{t}}$ is a morphism of varieties, and the variety $\Omega_{\mathfrak{t}}$ is irreducible with $\operatorname{dim} \Omega_{\mathfrak{t}}=\operatorname{dim}_{k} \mathfrak{g}-\operatorname{dim}_{k} C_{\mathfrak{g}}(\mathfrak{t})$.

(2) If $\mathfrak{t}$ is a maximal torus, then $\mathfrak{g}=\bigcup_{\mathfrak{h} \in \Omega_{\mathfrak{t}}} \mathfrak{h}$.

Proof. (1) Recall the Grassmann scheme $\operatorname{Gr}_{d}(\mathfrak{g}): M_{k} \longrightarrow$ Ens, i.e., the $k$-functor that assigns to every commutative $k$-algebra $R$ the set $\operatorname{Gr}_{d}(\mathfrak{g})(R)$ of $R$-direct summands of $\mathfrak{g} \otimes_{k} R$ of rank $d$ (cf. [16, (I.1.9)]). Let $A:=k\left[\mathcal{X}_{\mathfrak{t}}\right]$ be the function algebra of the affine scheme $\mathcal{X}_{\mathfrak{t}}$. As noted in Section 2, the $p$-subalgebra $\tilde{\mathfrak{g}}_{0} \subset \mathfrak{g} \otimes_{k} A$ is an element of $\operatorname{Gr}_{d}(\mathfrak{g})(A)$ for $d=\operatorname{dim}_{k} C_{\mathfrak{g}}(\mathfrak{t})$. By Yoneda's Lemma (cf. [16, (I.1.3)]) the point $\tilde{\mathfrak{g}}_{0}$ defines a natural transformation

$$
\lambda: \operatorname{Spec}_{k}(A) \longrightarrow \operatorname{Gr}_{d}(\mathfrak{g}) \quad ; \quad x \mapsto \operatorname{Gr}_{d}(\mathfrak{g})(x)\left[\tilde{\mathfrak{g}}_{0}\right]
$$

Thus, $\lambda_{R}(x)=\tilde{\mathfrak{g}}_{0} \otimes_{A} R$ with $R$ being viewed as an $A$-module via $x \in \operatorname{Spec}_{k}(A)(R)$. In view of [16, (I.1.11)] $\lambda_{k}=\zeta_{\mathfrak{t}}$ is a morphism $\mathcal{X}_{\mathfrak{t}}(k) \longrightarrow \operatorname{Gr}_{d}(\mathfrak{g})(k)$ of varieties. In particular, $\Omega_{\mathrm{t}}$ is irreducible.

Let $j: \mathfrak{t} \longrightarrow \tilde{\mathfrak{g}}$ be the universal embedding of $\mathfrak{t}$ relative to $\mathcal{X}_{\mathfrak{t}}$, and put $\varphi_{z}:=$ $\left(\operatorname{id}_{\mathfrak{g}} \otimes z\right) \circ j$ for every $z \in \mathcal{X}_{\mathfrak{t}}(k)$. Let $x \in \mathcal{X}_{\mathfrak{t}}(k)$. If $y \in \zeta_{\mathfrak{t}}^{-1}\left(\zeta_{\mathfrak{t}}(x)\right)$, then the $p$ subalgebras $\mathfrak{h}:=\tilde{\mathfrak{g}}_{0}(x)$ and $\tilde{\mathfrak{g}}_{0}(y)$ coincide. Consequently, $\varphi_{x}(\mathfrak{t}) \cup \varphi_{y}(\mathfrak{t}) \subset C(\mathfrak{h})$, so that $\varphi_{x}, \varphi_{y} \in \mathcal{T}_{C(\mathfrak{h})}(k)$. Since $C(\mathfrak{h})$ is abelian, the variety $\mathcal{T}_{C(\mathfrak{h})}(k)$ is finite. Thus, the fibre $\zeta_{\mathfrak{t}}^{-1}\left(\zeta_{\mathfrak{t}}(x)\right)$ is finite, and [11, (1.6)] now implies

$$
\operatorname{dim} \Omega_{\mathfrak{t}}=\operatorname{dim} \mathcal{X}_{\mathfrak{t}}(k)=\operatorname{dim}_{k} \mathfrak{g}-\operatorname{dim}_{k} C_{\mathfrak{g}}(\mathfrak{t}),
$$

as desired.

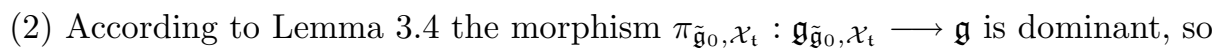
that the set $D:=\bigcup_{x \in \mathcal{X}_{\mathfrak{t}}(k)} \tilde{\mathfrak{g}}_{0}(x)$ lies dense in $\mathfrak{g}$. On the other hand, $\Omega_{\mathfrak{t}}^{\mathfrak{g}}$ is a closed subset of $\mathfrak{g} \times \operatorname{Gr}_{d}(\mathfrak{g})(k)$, and the projection $\pi_{\mathfrak{g}}: \Omega_{\mathfrak{t}}^{\mathfrak{g}} \longrightarrow \mathfrak{g}$ is closed. In particular, $\operatorname{im} \pi_{\mathfrak{g}}=\bigcup_{\mathfrak{h} \in \Omega_{\mathfrak{t}}} \mathfrak{h}$ is a closed subset of $\mathfrak{g}$ containing the dense subset $D$.

\section{Cartan subalgebras of Dimension $\operatorname{rk}(\mathfrak{g})$}

8.1. The variety $\mathfrak{C a r}(\mathfrak{g})$. In this section we are going to apply the results of $\S 6$ to study the variety $\mathfrak{C a r}(\mathfrak{g}):=\operatorname{Car}_{\operatorname{rk}(\mathfrak{g})}(\mathfrak{g})(k)$ of Cartan subalgebras of $\mathfrak{g}$ of minimal 
dimension. We begin by providing a characterization of these Cartan subalgebras in terms of the set $\Omega_{\mathrm{t}}$.

We denote by $T(\mathfrak{g})$ the toral radical of $\mathfrak{g}$, that is, the largest $p$-ideal of $\mathfrak{g}$ which is a torus. Note that $T(\mathfrak{g}) \subset C(\mathfrak{g})$ and that $T(\mathfrak{g})=S_{\mathfrak{g}}$ whenever $\mathfrak{g}$ is nilpotent (cf. [29, (II.4.2)]).

Lemma 8.1. Let $\mathfrak{h} \subset \mathfrak{g}$ be a Cartan subalgebra. Then the following statements are equivalent:

(1) $\operatorname{dim}_{k} \mathfrak{h}=\operatorname{rk}(\mathfrak{g})$

(2) $\Omega_{T(\mathfrak{h})} \subset \operatorname{Nil}_{\operatorname{rk}(\mathfrak{g})}(\mathfrak{g})(k)$.

(3) $\tilde{\mathfrak{g}}_{0}(x)$ is nilpotent for every $x \in \mathcal{X}_{T(\mathfrak{h})}(k)$.

Proof. $(1) \Rightarrow(2)$ Owing to $3.5(3))$ there exists a dense open set $O \subset \mathcal{X}_{T(\mathfrak{h})}(k)$ such that $\mu\left(\tilde{\mathfrak{g}}_{0}(x)\right)=\mu(\mathfrak{g})$ for every $x \in O$. Given $x \in O$, we can thus find a torus $\mathfrak{t}_{x}$ of dimension $\mu(\mathfrak{g})$ with $\varphi_{x}(\mathfrak{t}) \subset \mathfrak{t}_{x}$, so that $C_{\mathfrak{g}}\left(\mathfrak{t}_{x}\right) \subset C_{\mathfrak{g}}\left(\varphi_{x}(\mathfrak{t})\right)=\tilde{\mathfrak{g}}_{0}(x)$. Observing (3.5) (1)) we obtain

$$
\operatorname{rk}(\mathfrak{g})=\operatorname{dim}_{k} C_{\mathfrak{g}}\left(\mathfrak{t}_{x}\right) \leq \operatorname{dim}_{k} \tilde{\mathfrak{g}}_{0}(x)=\operatorname{dim}_{k} \mathfrak{h}=\operatorname{rk}(\mathfrak{g}),
$$

proving that $\tilde{\mathfrak{g}}_{0}(x)=C_{\mathfrak{g}}\left(\mathfrak{t}_{x}\right)$ is nilpotent. In view of (7.2) and (7.7) the set $C$, consisting of those elements $x$ of $\mathcal{X}_{T(\mathfrak{h})}(k)$ such that $\tilde{\mathfrak{g}}_{0}(x)$ is nilpotent, is closed. As $O \subset C$, we obtain $C=\mathcal{X}_{T(\mathfrak{h})}(k)$. Thus, $\operatorname{im} \zeta_{T(\mathfrak{h})} \subset \operatorname{Nil} \operatorname{rk}(\mathfrak{g})(\mathfrak{g})(k)$, and another application of (7.2) yields the assertion.

(2) $\Rightarrow(3)$ Trivial

$(3) \Rightarrow(1)$ Thanks to $(3.5)$ (3) there exists an element $x_{0} \in \mathcal{X}_{T(\mathfrak{h})}(k)$ such that $\mu\left(\tilde{\mathfrak{g}}_{0}\left(x_{0}\right)\right)=\mu(\mathfrak{g})$. As $\tilde{\mathfrak{g}}_{0}\left(x_{0}\right)$ is nilpotent, it is a Cartan subalgebra and thus equal to $C_{\mathfrak{g}}\left(T\left(\tilde{\mathfrak{g}}_{0}\left(x_{0}\right)\right)\right)$. Since $\operatorname{dim}_{k} T\left(\tilde{\mathfrak{g}}_{0}\left(x_{0}\right)\right)=\mu(\mathfrak{g})$, (1) of (3.5) implies

$$
\operatorname{dim}_{k} \mathfrak{h}=\operatorname{dim}_{k} \tilde{\mathfrak{g}}_{0}\left(x_{0}\right)=\operatorname{rk}(\mathfrak{g}),
$$

as desired.

Let $\mathfrak{t} \subset \mathfrak{g}$ be a torus of dimension $\mu(\mathfrak{g})$. By the arguments of $(7.7(1))$ the map

$$
\mathcal{X}_{\mathfrak{t}}(k) \longrightarrow \operatorname{Tor}_{\mu(\mathfrak{g})}(\mathfrak{g})(k) ; \varphi \mapsto \varphi(\mathfrak{t})
$$

is a morphism of varieties. Owing to (4.1(1)) this map is surjective with finite fibres. In view of [11, (1.6)] and $(3.5(1))$ the variety $\operatorname{Tor}_{\mu(\mathfrak{g})}(\mathfrak{g})(k)$ is irreducible of $\operatorname{dimension} \operatorname{dim}_{k} \mathfrak{g}-\operatorname{rk}(\mathfrak{g})$. The following result establishes analogous properties for the variety $\mathfrak{C a r}(\mathfrak{g})$ of Cartan subalgebras of dimension $\operatorname{rk}(\mathfrak{g})$. By Corollary 4.4 both varieties are homogeneous $\mathcal{G}(k)$-spaces in case $\mathfrak{g}=\operatorname{Lie}(\mathcal{G})$ is the Lie algebra of a smooth connected group $\mathcal{G}$. In particular, $\operatorname{Tor}_{\mu(\mathfrak{g})}(\mathfrak{g})(k)$ and $\mathfrak{C a r}(\mathfrak{g})$ are smooth and irreducible in the classical case.

Theorem 8.2. Let $(\mathfrak{g},[p])$ be a finite-dimensional restricted Lie algebra.

(1) We have $\Omega_{\mathfrak{t}}=\overline{\mathfrak{C a} \mathfrak{r}(\mathfrak{g})}$ for every torus $\mathfrak{t} \in \operatorname{Tor}_{\mu(\mathfrak{g})}(\mathfrak{g})(k)$.

(2) The variety $\mathfrak{C a r}(\mathfrak{g})$ is irreducible of dimension $\operatorname{dim}_{k} \mathfrak{g}-\operatorname{rk}(\mathfrak{g})$.

(3) Let $\mathfrak{t}$ be a torus of dimension $\mu(\mathfrak{g})$. Then $\operatorname{im} \zeta_{\mathfrak{t}}=\{\mathfrak{h} \in \mathfrak{C a r}(\mathfrak{g}) ; \mu(\mathfrak{h})=\mu(\mathfrak{g})\}$ is a dense, open subset of $\mathfrak{C a r}(\mathfrak{g})$.

Proof. Let $\mathfrak{t} \subset \mathfrak{g}$ be a torus of dimension $\mu(\mathfrak{g})$. By virtue of (3.5)(1)) the centralizer $\mathfrak{h}:=C_{\mathfrak{g}}(\mathfrak{t})$ is a Cartan subalgebra of $\operatorname{dimension} \operatorname{rk}(\mathfrak{g})$ with $T(\mathfrak{h})=\mathfrak{t}$. Thanks to (8.1) we thus have $\Omega_{\mathfrak{t}} \subset \operatorname{Nil}_{\operatorname{rk}(\mathfrak{g})}(\mathfrak{g})(k)$.

Let $\mathfrak{h}$ be a Cartan subalgebra of dimension $\operatorname{rk}(\mathfrak{g})$. By (3.2) there exists a semisimple element $s \in S_{\mathfrak{g}}$ such that $\mathfrak{h}=C_{\mathfrak{g}}(s)$. In view of $(7.7(2))$ we can find $\mathfrak{s} \in \Omega_{\mathfrak{t}}$ 
containing $s$. As $\mathfrak{s}$ is nilpotent, we have $\mathfrak{s} \subset \mathfrak{h}$, whence $\mathfrak{h}=\mathfrak{s}$ by equality of dimensions. Thanks to $[8.1(3))$ we now obtain

$$
\operatorname{im} \zeta_{\mathfrak{t}} \subset \mathfrak{C a r}(\mathfrak{g}) \subset \Omega_{\mathfrak{t}} .
$$

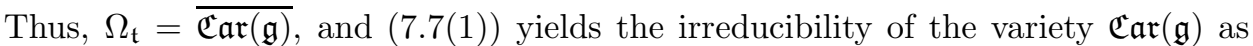
well as $\operatorname{dim} \mathfrak{C a r}(\mathfrak{g})=\operatorname{dim} \Omega_{\mathfrak{t}}=\operatorname{dim}_{k} \mathfrak{g}-\operatorname{rk}(\mathfrak{g})$. This completes the proof of (1) and (2).

(3) Let $d \geq 0$. Since $N(\mathfrak{g})$ is conical with $N(\mathfrak{g}) \cap \mathfrak{h}=N(\mathfrak{h})$ for every $\mathfrak{h} \in$ $\operatorname{Sub}_{\operatorname{rk}(\mathfrak{g})}(\mathfrak{g})(k)$, Lemma 7.3 shows that

$$
U_{d}:=\{\mathfrak{h} \in \mathfrak{C a r}(\mathfrak{g}) ; \operatorname{dim} N(\mathfrak{h}) \leq d\}
$$

is an open subset of $\mathfrak{C a r}(\mathfrak{g})$. As semisimple elements lie centrally in nilpotent algebras, the Jordan-Chevalley decomposition readily yields $\operatorname{rk}(\mathfrak{g})=\operatorname{dim} N(\mathfrak{h})+$ $\mu(\mathfrak{h})$ for every $\mathfrak{h} \in \mathfrak{C} \mathfrak{a r}(\mathfrak{g})$. Consequently,

$$
U_{\operatorname{rk}(\mathfrak{g})-\mu(\mathfrak{g})}=\{\mathfrak{h} \in \mathfrak{C a r}(\mathfrak{g}) ; \mu(\mathfrak{h})=\mu(\mathfrak{g})\}
$$

is a dense, open subset of the irreducible variety $\mathfrak{C a r}(\mathfrak{g})$. The identity $U_{\operatorname{rk}(\mathfrak{g})-\mu(\mathfrak{g})}=$ $\operatorname{im} \zeta_{\mathfrak{t}}$ is a direct consequence of $\left.4.1(1)\right)$.

8.2. Generic properties. The group $\operatorname{Aut}_{p}(\mathfrak{g})$ of automorphisms of the restricted Lie algebra $\mathfrak{g}$ operates on the variety $\operatorname{Car}_{\mathrm{rk}(\mathfrak{g})}(\mathfrak{g})(k)$. If this action affords only finitely many orbits, then (8.2) provides a dense, open orbit, so that generically all Cartan subalgebras of minimal dimension have the same structure. Demuškin [7, 8] has shown that the automorphism group acts with finitely many orbits for each of the simple Lie algebras of Cartan type.

In view of (3.6) Cartan subalgebras are also generically isomorphic whenever the underlying Lie algebra admits a toral Cartan subalgebra. We now turn to the general case and study the ascending central series of Cartan subalgebras. Given a finite-dimensional restricted Lie algebra $(\mathfrak{g},[p])$ and $m \in \mathbb{N}_{0}$, we consider the conical variety

$$
\mathcal{V}_{\mathfrak{g}}^{m}:=\left\{x \in \mathfrak{g} ; x^{[p]^{m}}=0\right\} .
$$

Thus, we have $\mathcal{V}_{\mathfrak{g}}^{0}=\{0\}, \mathcal{V}_{\mathfrak{g}}^{1}=\mathcal{V}_{\mathfrak{g}}$ as well as $\mathcal{V}_{\mathfrak{g}}^{m}=N(\mathfrak{g})$ for $m=\operatorname{dim}_{k} \mathfrak{g}$. Recall that the ascending central series $\left(C_{n}(\mathfrak{g})\right)_{n \geq 0}$ of $\mathfrak{g}$ is inductively defined via

$$
C_{0}(\mathfrak{g}):=(0) \quad \text { and } \quad C_{n+1}(\mathfrak{g}):=\left\{x \in \mathfrak{g} ;[\mathfrak{g}, x] \subset C_{n}(\mathfrak{g})\right\} .
$$

In other words,

$$
C_{n}(\mathfrak{g})=\left\{x \in \mathfrak{g} ;\left(\operatorname{ad} y_{1}\right) \circ\left(\operatorname{ad} y_{2}\right) \circ \cdots \circ\left(\operatorname{ad} y_{n}\right)(x)=0 \quad \forall y_{1}, \ldots, y_{n} \in \mathfrak{g}\right\} .
$$

Note that each $C_{n}(\mathfrak{g})$ is a $p$-ideal of $\mathfrak{g}$. We put $C_{-1}(\mathfrak{g}):=(0)$.

Lemma 8.3. Let $(\mathfrak{g},[p])$ be a finite-dimensional restricted Lie algebra. If $\operatorname{Sub}_{d}(\mathfrak{g})(k)$ $\neq \emptyset$, then there exist $r_{m, n} \in \mathbb{N}_{0}$ for all $m, n \in \mathbb{N}_{0}$ such that

(a) $O_{m, n}:=\left\{\mathfrak{h} \in \operatorname{Sub}_{d}(\mathfrak{g})(k) ; \operatorname{dim} \mathcal{V}_{C_{n}(\mathfrak{h}) / C_{n-1}(\mathfrak{h})}^{m}+\operatorname{dim}_{k} C_{n-1}(\mathfrak{h})=r_{m, n}\right\}$ is a nonempty open subset of $\operatorname{Sub}_{d}(\mathfrak{g})(k)$, and

(b) $\operatorname{dim} \mathcal{V}_{C_{n}(\mathfrak{h}) / C_{n-1}(\mathfrak{h})}^{m}+\operatorname{dim}_{k} C_{n-1}(\mathfrak{h}) \geq r_{m, n}$ for every $\mathfrak{h} \in \operatorname{Sub}_{d}(\mathfrak{g})(k)$.

Proof. For $q \geq 1$ we consider the closed subset

$$
\tilde{\Sigma}_{q}:=\left\{\left(\mathfrak{h}, y_{1}, \ldots, y_{q}\right) \in \operatorname{Sub}_{d}(\mathfrak{g})(k) \times \mathfrak{g}^{q} ;\left(y_{1}, \ldots, y_{q}\right) \in \mathfrak{h}^{q}\right\}
$$

of $\operatorname{Sub}_{d}(\mathfrak{g})(k) \times \mathfrak{g}^{q}$. Since the set

$$
X:=\left\{(f, \mathfrak{h}, x) \in \operatorname{End}_{k}(\mathfrak{g}) \times \tilde{\Sigma}_{1} ; f(x)=0\right\}
$$


is closed in $\operatorname{End}_{k}(\mathfrak{g}) \times \tilde{\Sigma}_{1}$, its inverse image $Y \subset \tilde{\Sigma}_{n+1}$ under the morphism $\tilde{\Sigma}_{n+1} \longrightarrow \operatorname{End}_{k}(\mathfrak{g}) \times \tilde{\Sigma}_{1} ;\left(\mathfrak{h}, y_{1}, \ldots, y_{n}, x\right) \mapsto\left(\left(\operatorname{ad} y_{1}\right) \circ\left(\operatorname{ad} y_{2}\right) \circ \cdots \circ\left(\operatorname{ad} y_{n}\right), \mathfrak{h}, x\right)$ is also closed. Consider the projection

$$
\pi: Y \longrightarrow \tilde{\Sigma}_{1} ; \quad\left(\mathfrak{h}, y_{1}, \ldots, y_{n}, x\right) \mapsto(\mathfrak{h}, x) .
$$

As

$\pi^{-1}\left(\pi\left(\mathfrak{h}, y_{1}, \ldots, y_{n}, x\right)\right) \cong\left\{\left(a_{1}, \ldots, a_{n}\right) \in \mathfrak{h}^{n} ;\left(\operatorname{ad} a_{1}\right) \circ\left(\operatorname{ad} a_{2}\right) \circ \cdots \circ\left(\operatorname{ad} a_{n}\right)(x)=0\right\}$

is a conical subvariety of $\mathfrak{h}^{n}$, (1.1) implies the upper semicontinuity of the map

$\tilde{\Sigma}_{1} \longrightarrow \mathbb{N}_{0} ;(\mathfrak{h}, x) \mapsto \operatorname{dim}\left\{\left(a_{1}, \ldots, a_{n}\right) \in \mathfrak{h}^{n} ;\left(\operatorname{ad} a_{1}\right) \circ\left(\operatorname{ad} a_{2}\right) \circ \cdots \circ\left(\operatorname{ad} a_{n}\right)(x)=0\right\}$.

In particular,

$$
\begin{aligned}
Z_{n} & :=\left\{(\mathfrak{h}, x) \in \tilde{\Sigma}_{1} ;\left(\operatorname{ad} y_{1}\right) \circ\left(\operatorname{ad} y_{2}\right) \circ \cdots \circ\left(\operatorname{ad} y_{n}\right)(x)=0 \text { for all } y_{1}, \ldots, y_{n} \in \mathfrak{h}\right\} \\
& =\left\{(\mathfrak{h}, x) \in \tilde{\Sigma}_{1} ; x \in C_{n}(\mathfrak{h})\right\}
\end{aligned}
$$

is a closed subset of $\tilde{\Sigma}_{1}$. Moreover, for each $m \in \mathbb{N}_{0}$, the set

$$
Z_{n}^{m}:=\left\{(\mathfrak{h}, x) \in Z_{n} ;\left(\mathfrak{h}, x^{[p]^{m}}\right) \in Z_{n-1}\right\}
$$

is a closed subvariety of $Z_{n}$. If $\gamma: Z_{n}^{m} \longrightarrow \operatorname{Sub}_{d}(\mathfrak{g})(k)$ denotes the projection onto the first factor, then

$$
\gamma^{-1}\left(\gamma\left(\mathfrak{h}, x_{0}\right)\right) \cong\left\{x \in C_{n}(\mathfrak{h}) ; x^{[p]^{m}} \in C_{n-1}(\mathfrak{h})\right\} .
$$

Since the factor algebra $C_{n}(\mathfrak{h}) / C_{n-1}(\mathfrak{h})$ is abelian, the latter variety is readily seen to be a subspace of $C_{n}(\mathfrak{h})$ of dimension $\operatorname{dim} \mathcal{V}_{C_{n}(\mathfrak{h}) / C_{n-1}(\mathfrak{h})}^{m}+\operatorname{dim}_{k} C_{n-1}(\mathfrak{h})$. It follows that the map

$$
\operatorname{Sub}_{d}(\mathfrak{g})(k) \longrightarrow \mathbb{N}_{0} ; \mathfrak{h} \longrightarrow \operatorname{dim} \mathcal{V}_{C_{n}(\mathfrak{h}) / C_{n-1}(\mathfrak{h})}^{m}+\operatorname{dim}_{k} C_{n-1}(\mathfrak{h})
$$

is upper semicontinuous, so that

$$
r_{m, n}:=\min \left\{\operatorname{dim} \mathcal{V}_{C_{n}(\mathfrak{h}) / C_{n-1}(\mathfrak{h})}^{m}+\operatorname{dim}_{k} C_{n-1}(\mathfrak{h}) ; \mathfrak{h} \in \operatorname{Sub}_{d}(\mathfrak{g})(k)\right\}
$$

has the requisite properties.

Our next result supports the following conjecture on generic stability of the structure of Cartan subalgebras of minimal dimension.

Conjecture. Let $(\mathfrak{g},[p])$ be a finite-dimensional restricted Lie algebra. Then there exists a dense, open subset $O \subset \mathfrak{C a r}(\mathfrak{g})$ such that $\mathfrak{h}_{1} \cong \mathfrak{h}_{2}$ for any $\mathfrak{h}_{1}, \mathfrak{h}_{2} \in O$.

Theorem 8.4. Let $(\mathfrak{g},[p])$ be a finite-dimensional restricted Lie algebra. There exists a dense, open subset $O \subset \mathfrak{C a r}(\mathfrak{g})$ such that for any $n \in\{1, \ldots, \operatorname{rk}(\mathfrak{g})\}$ and $\mathfrak{h}_{1}, \mathfrak{h}_{2} \in O$ we have $C_{n}\left(\mathfrak{h}_{1}\right) / C_{n-1}\left(\mathfrak{h}_{1}\right) \cong C_{n}\left(\mathfrak{h}_{2}\right) / C_{n-1}\left(\mathfrak{h}_{2}\right)$.

Proof. Letting $\mathfrak{n}_{i}$ denote the nil-cyclic restricted Lie algebra of dimension $i$, we observe that

$$
\operatorname{dim} \mathcal{V}_{\mathfrak{n}_{i}}^{m}-\operatorname{dim} \mathcal{V}_{\mathfrak{n}_{i}}^{m-1}= \begin{cases}1 & \text { if } m \leq i, \\ 0 & \text { if } m>i,\end{cases}
$$

for every $m \geq 1$. If $\mathfrak{h}$ is an abelian $p$-subalgebra of $\mathfrak{g}$, then [6, $(\mathrm{IV}, \S 2,2.14)]$ implies that

$$
\mathfrak{h} \cong T(\mathfrak{h}) \oplus \bigoplus_{i=1}^{\ell} s_{i} \mathfrak{n}_{i}, \quad \ell, s_{i} \in \mathbb{N}_{0}
$$


Consequently,

$$
\operatorname{dim} \mathcal{V}_{\mathfrak{h}}^{m}-\operatorname{dim} \mathcal{V}_{\mathfrak{h}}^{m-1}=\sum_{i=1}^{\ell} s_{i}\left(\operatorname{dim} \mathcal{V}_{\mathfrak{n}_{i}}^{m}-\operatorname{dim} \mathcal{V}_{\mathfrak{n}_{i}}^{m-1}\right)=\sum_{i \geq m} s_{i}
$$

is the number of nil-cyclic components of dimension greater than or equal to $m$. Accordingly, the isomorphism type of $\mathfrak{h}$ is completely determined by the tuple $\left(\operatorname{dim}_{k} \mathfrak{h}, \operatorname{dim} \mathcal{V}_{\mathfrak{h}}^{1}, \ldots, \operatorname{dim} \mathcal{V}_{\mathfrak{h}}^{q}\right)$, where $q:=\operatorname{dim}_{k} \mathfrak{h}$.

In virtue of the obvious generalization of (8.3) there exist, for $m, n \geq 0$, natural numbers $r_{m, n} \in \mathbb{N}_{0}$ such that

$$
O_{m, n}:=\left\{\mathfrak{h} \in \mathfrak{C a r}(\mathfrak{g}) ; \operatorname{dim} \mathcal{V}_{C_{n}(\mathfrak{h}) / C_{n-1}(\mathfrak{h})}^{m}+\operatorname{dim}_{k} C_{n-1}(\mathfrak{h})=r_{m, n}\right\}
$$

is a nonempty open subset of $\mathfrak{C a r}(\mathfrak{g})$. Thanks to $[8.2(2))$ the open set

$$
O:=\bigcap_{m=0}^{\mathrm{rk}(\mathfrak{g})} \bigcap_{n=1}^{\mathrm{rk}(\mathfrak{g})} O_{m, n}
$$

lies dense in $\mathfrak{C a r}(\mathfrak{g})$. Let $\mathfrak{h}$ be an element of $O$. Since $\mathfrak{h} \in O_{0, n}$ for all $n$, it follows that $\operatorname{dim}_{k} C_{n-1}(\mathfrak{h})=r_{0, n}$. Thus, the containment $\mathfrak{h} \in O_{m, n}$ entails $\operatorname{dim} \mathcal{V}_{C_{n}(\mathfrak{h}) / C_{n-1}(\mathfrak{h})}^{m}=$ $r_{m, n}-r_{0, n}=: s_{m, n}$ for all $m \in\{0, \ldots, \operatorname{rk}(\mathfrak{g})\}, n \in\{1, \ldots, \operatorname{rk}(\mathfrak{g})\}$. In view of our above observations, the identity

$$
\begin{aligned}
& \left(\operatorname{dim}_{k} C_{n}(\mathfrak{h}) / C_{n-1}(\mathfrak{h}), \operatorname{dim} \mathcal{V}_{C_{n}(\mathfrak{h}) / C_{n-1}(\mathfrak{h})}, \ldots, \operatorname{dim} \mathcal{V}_{C_{n}(\mathfrak{h}) / C_{n-1}(\mathfrak{h})}^{\mathrm{rk}(\mathfrak{g})}\right) \\
& \quad=\left(r_{0, n+1}-r_{0, n}, s_{1, n}, \ldots, s_{\operatorname{rk}(\mathfrak{g}), n}\right)
\end{aligned}
$$

shows that $O$ has the desired properties.

Remark. If $\mathfrak{C a r}(\mathfrak{g}) \subset \operatorname{Ab}_{\operatorname{rk}(\mathfrak{g})}(\mathfrak{g})(k)$, then the Cartan subalgebras of dimension $\operatorname{rk}(\mathfrak{g})$ are generically isomorphic. The following observation slightly generalizes our earlier remark on self-centralizing tori:

If $\mathfrak{h} \subset \mathfrak{g}$ is a Cartan subalgebra such that $\operatorname{dim} \mathcal{V}_{\mathfrak{h}} \leq 1$, then every Cartan subalgebra of dimension $\mathrm{rk}(\mathfrak{g})$ is abelian.

Let $\mathfrak{t} \subset \mathfrak{g}$ be a maximal torus such that $\mathfrak{h}=C_{\mathfrak{g}}(\mathfrak{t})$. According to (1.6)

$$
O_{\mathfrak{t}}:=\left\{x \in \mathcal{X}_{\mathfrak{t}}(k) ; \operatorname{dim} \mathcal{V}_{\tilde{\mathfrak{g}}_{0}(x)} \leq 1\right\}
$$

is a dense, open subset of $\mathcal{X}_{\mathfrak{t}}(k)$. Theorem 3.5 3 ) provides an element $x_{0} \in O_{\mathfrak{t}}$ such that $\mu\left(\tilde{\mathfrak{g}}_{0}(x)\right)=\mu(\mathfrak{g})$. Hence there exists a torus $\mathfrak{t}_{0} \subset \mathfrak{g}$ of dimension $\mu(\mathfrak{g})$ such that $\varphi_{x_{0}}(\mathfrak{t}) \subset \mathfrak{t}_{0}$. Owing to (3.5)(1)) $\mathfrak{h}_{0}:=C_{\mathfrak{g}}\left(\mathfrak{t}_{0}\right)$ is a Cartan subalgebra of dimension $\operatorname{rk}(\mathfrak{g})$, which also satisfies $\operatorname{dim} \mathcal{V}_{\mathfrak{h}_{0}} \subset \operatorname{dim} \mathcal{V}_{\tilde{\mathfrak{g}}_{0}\left(x_{0}\right)} \leq 1$. It now follows from (7.3), and (8.2) that

$$
U:=\left\{\mathfrak{h} \in \mathfrak{C a r}(\mathfrak{g}) ; \operatorname{dim} \mathcal{V}_{\mathfrak{h}} \leq 1\right\}
$$

is a dense, open subset of $\mathfrak{C a r}(\mathfrak{g})$. According to [12, (3.1)] each element of $U$ is abelian. Since the set of abelian $p$-subalgebras of $\operatorname{dimension} \operatorname{rk}(\mathfrak{g})$ is closed, our assertion follows.

Corollary 8.5. Let $(\mathfrak{g},[p])$ be a finite-dimensional restricted Lie algebra.

(1) There exists a dense open set $O \subset \mathfrak{C a r}(\mathfrak{g})$ such that any two elements of $O$ have the same generic nilpotency class $n_{0}$. Moreover, we have $\mathfrak{h}^{\left(n_{0}+1\right)}=(0)$ for every $\mathfrak{h} \in \mathfrak{C} \mathfrak{a r}(\mathfrak{g})$.

(2) Every Cartan subalgebra of dimension $\operatorname{rk}(\mathfrak{g})$ has nilpotency class $\leq \operatorname{rk}(\mathfrak{g})-$ $\mu(\mathfrak{g})+1$. 
Proof. (1) Thanks to (8.2) and (8.3) there exist natural numbers $r_{n} \in \mathbb{N}_{0}$ such that

$$
O_{n}:=\left\{\mathfrak{h} \in \mathfrak{C a r}(\mathfrak{g}), \operatorname{dim}_{k} C_{n}(\mathfrak{h})=r_{n}\right\}
$$

is a dense, open subset of $\mathfrak{C a r}(\mathfrak{g})$. Thus, $O_{n} \cap O_{n+1} \neq \emptyset$, so that $r_{n} \leq r_{n+1}$. Let $n_{0}:=\min \left\{n \geq 0 ; r_{n}=\operatorname{rk}(\mathfrak{g})\right\}$. Then we have $C_{n_{0}}(\mathfrak{h})=\mathfrak{h}$ for every element of $O_{n_{0}}$, and the elements of $O_{n_{0}}$ have nilpotency class $n_{0}$. Since $\operatorname{Nil}_{\mathrm{rk}(\mathfrak{g})}^{n_{0}}(\mathfrak{g})(k)$ is closed, the second statement also follows.

(2) Let $\mathfrak{h} \in \mathfrak{C a r}(\mathfrak{g})$ such that $\operatorname{dim}_{k} T(\mathfrak{h})=\mu(\mathfrak{g})$. Since $\mathfrak{h} / T(\mathfrak{h})$ is nilpotent of dimension $\operatorname{rk}(\mathfrak{g})-\mu(\mathfrak{g})$, we have $\mathfrak{h}^{(\operatorname{rk}(\mathfrak{g})-\mu(\mathfrak{g})+1)} \subset T(\mathfrak{h})$. Thus, $\mathfrak{h}^{(\operatorname{rk}(\mathfrak{g})-\mu(\mathfrak{g})+2)}=(0)$, so that $\mathfrak{h}$ has nilpotency class $\leq \ell:=\operatorname{rk}(\mathfrak{g})-\mu(\mathfrak{g})+1$. It follows that $\operatorname{im} \zeta_{T(\mathfrak{h})}$ is contained in the closed subset $\mathrm{Nil}_{\mathrm{rk}(\mathfrak{g})}^{\ell}(\mathfrak{g})(k)$ of nilpotent $p$-subalgebras of nilpotency class $\leq \ell$. In view of $(8.2(1))$ we thus have $\mathfrak{C a r}(\mathfrak{g}) \subset \Omega_{\mathfrak{t}} \subset \mathrm{Nil}_{\operatorname{rk}(\mathfrak{g})}^{\ell}(\mathfrak{g})(k)$, as desired.

The estimate given in [8.5(2)) is rather rough. By results of Demuškin [7] all Cartan subalgebras of the Cartan type algebra $S(n ; \underline{1})$ (cf. [29. (IV.3)]), the socalled special algebra, are abelian and of dimension $\operatorname{rk}(\overline{\bar{S}}(n ; \underline{1}))=(n-1)(p-1)$. On the other hand, each maximal torus of $S(n ; \underline{\underline{1}})$ has dimension $\mu(S(n ; \underline{\underline{1}}))=n-1$.

8.3. Deformations. The foregoing results raise the problem of determining the structure of the elements of the variety $\overline{\mathfrak{C a r}(\mathfrak{g})}$. For instance, if $\mathfrak{g}$ admits a selfcentralizing torus, then our variety consists entirely of abelian algebras. We shall see in $\overline{8.7}(2))$ below that $\overline{\mathfrak{C a r}(\mathfrak{g})}$ also contains $p$-nilpotent algebras in case $\mathfrak{g}=\operatorname{Lie}(\mathcal{G})$ is the Lie algebra of a smooth group $\mathcal{G}$ with $T(\mathfrak{g})=(0)$. The structure of the deformations on the boundary is in part determined by the number

$$
\mu_{\min }(\mathfrak{g}):=\min \{\mu(\mathfrak{h}) ; \mathfrak{h} \in \overline{\mathfrak{C a r}(\mathfrak{g})}\},
$$

which is also related to the degree of nilpotence of the $p$-nilpotent elements of $\mathfrak{g}$.

Corollary 8.6. The following statements hold:

(1) Every Cartan subalgebra of $\mathfrak{g}$ contains a p-subalgebra belonging to $\overline{\mathfrak{C a r}(\mathfrak{g})}$.

(2) $N(\mathfrak{g})^{[p]^{\mathrm{rk}(\mathfrak{g})-\mu_{\min }(\mathfrak{g})}}=\{0\}$.

(3) $\operatorname{dim}_{k}(k x)_{p} \leq \operatorname{rk}(\mathfrak{g})$ for every $x \in \mathfrak{g}$.

(4) If there exists an element $x_{0} \in \mathfrak{g}$ such that $\operatorname{dim}_{k}\left(k x_{0}\right)_{p}=\operatorname{rk}(\mathfrak{g})$, then $\overline{\mathfrak{C a r}(\mathfrak{g})} \subset \operatorname{Ab}_{\mathrm{rk}(\mathfrak{g})}(\mathfrak{g})(k)$.

(5) If there is $x_{0} \in N(\mathfrak{g})$ such that $x_{0}^{[p]^{\mathrm{rk}(\mathfrak{g})-\mu_{\min }(\mathfrak{g})-1}} \neq 0$, then $\overline{\mathfrak{C a r}(\mathfrak{g})} \subset$ $\operatorname{Ab}_{\operatorname{rk}(\mathfrak{g})}(\mathfrak{g})(k)$.

Proof. (1) Let $\mathfrak{h} \subset \mathfrak{g}$ be a Cartan subalgebra. In view of (3.2) we can find a semisimple element $s \in S_{\mathfrak{g}}$ such that $\mathfrak{h}=C_{\mathfrak{g}}(s)$. By $[8.2(1))$ and $(\mathbf{7 . 7}(2))$ the element $s$ is contained in a suitably chosen nilpotent $p$-subalgebra $\mathfrak{n} \in \overline{\mathfrak{C a r}(\mathfrak{g})}$. As $s$ is semisimple, we have $s \in C(\mathfrak{n})$, whence $\mathfrak{n} \subset C_{\mathfrak{g}}(s)=\mathfrak{h}$.

(2) Let $x$ be an element of $N(\mathfrak{g})$. Owing to $(8.2(1))$ and $\mathbb{8 7 . 7}(2))$ there exists $\mathfrak{h} \in \overline{\mathfrak{C a r}(\mathfrak{g})}$ such that $x \in N(\mathfrak{h})$. Since the factor algebra $\mathfrak{h} / T(\mathfrak{h})$ of the nilpotent algebra $\mathfrak{h}$ is $p$-nilpotent and of dimension $\operatorname{rk}(\mathfrak{g})-\mu(\mathfrak{h})$, we obtain $x^{[p]^{\operatorname{rk}(\mathfrak{g})-\mu(\mathfrak{h})}} \in$ $N(\mathfrak{h}) \cap T(\mathfrak{h})=\{0\}$. As $\mu(\mathfrak{h}) \geq \mu_{\min }(\mathfrak{g})$, our assertion follows.

(3) According to $\mathbf{7 . 7}(2))$ every element of $\mathfrak{g}$ is contained in a $p$-subalgebra of dimension $\operatorname{rk}(\mathfrak{g})$. 
(4),(5) Thanks to $\overline{8.2(1))}$ and $\overline{7.7}(2))$ there exists $\mathfrak{h}_{0} \in \overline{\mathfrak{C a r}(\mathfrak{g})}$ such that $x_{0} \in \mathfrak{h}_{0}$. In case $(5)$ we have $\left(k x_{0}\right)_{p} \cap T\left(\mathfrak{h}_{0}\right)=(0)$, so that $\operatorname{dim}_{k}\left(\left(k x_{0}\right)_{p} \oplus T\left(\mathfrak{h}_{0}\right)\right) \geq \operatorname{rk}(\mathfrak{g})-$ $\mu_{\min }(\mathfrak{g})+\operatorname{dim}_{k} T\left(\mathfrak{h}_{0}\right) \geq \operatorname{rk}(\mathfrak{g})$. Consequently,

$$
\mathfrak{h}_{0}=\left(k x_{0}\right)_{p} \oplus T\left(\mathfrak{h}_{0}\right),
$$

whence $\operatorname{dim} \mathcal{V}_{\mathfrak{h}_{0}} \leq 1$.

Alternatively, $\mathfrak{h}_{0}=\left(k x_{0}\right)_{p}$ and the abelian Lie algebra $\mathfrak{h}_{0}$ decomposes into a direct sum $\mathfrak{h}_{0}=S_{\mathfrak{h}_{0}} \oplus N\left(\mathfrak{h}_{0}\right)$ of restricted Lie algebras. Consequently, $N\left(\mathfrak{h}_{0}\right)$ is generated by the nilpotent part of $x_{0}$, and $\operatorname{dim} \mathcal{V}_{\mathfrak{h}_{0}} \leq 1$.

In view of $(8.2(2))$ and (7.3) the set

$$
O:=\left\{\mathfrak{h} \in \overline{\mathfrak{C} \mathfrak{a r}(\mathfrak{g})} ; \operatorname{dim} \mathcal{V}_{\mathfrak{h}} \leq 1\right\}
$$

lies dense in $\overline{\mathfrak{C a r}(\mathfrak{g})}$. Thanks to [12, (3.1)] the set $O$ is contained in $\operatorname{Ab}_{\operatorname{rk}(\mathfrak{g})}(\mathfrak{g})(k)$, and we may now apply (7.2) to obtain the desired result.

Remark. Let $\mathfrak{h} \subset \mathfrak{g}$ be an arbitrary Cartan subalgebra. By (8.6) there exists a $p$-subalgebra $\mathfrak{n} \in \overline{\mathfrak{C} \mathfrak{a r}(\mathfrak{g})}$ such that $\mathfrak{n} \subset \mathfrak{h}$. Hence we have $\mu_{\min }(\mathfrak{g}) \leq \mu(\mathfrak{n}) \leq \mu(\mathfrak{h})$, so that

$$
\mu_{\min }(\mathfrak{g}) \leq \min \{\mu(\mathfrak{h}) ; \mathfrak{h} \text { Cartan subalgebra of } \mathfrak{g}\}
$$

This inequality is usually strict (cf. (8.7) below).

Example. Let $W(1, m)$ be the $p^{m}$-dimensional Zassenhaus algebra. For $p \geq 3$ this algebra is simple, and it is restricted if and only if $m=1$. By construction, $W(1, m) \subset \operatorname{Der}_{k}(A(1, m))$ is a subalgebra of the restricted Lie algebra of derivations of the commutative $k$-algebra $A(1, m)=\bigoplus_{i=0}^{p^{m}-1} k x^{(i)}$ of "divided powers" (cf. [29, (IV.2)]). (The algebra $A(1, m)$ can also be viewed as the distribution algebra $H\left(\alpha_{p^{m}}\right)$ of the $m$-th Frobenius kernel of the additive group $\alpha_{k}:=\operatorname{Spec}_{k}(k[T])$.) If $D$ denotes the derivation defined by $D\left(x^{(i)}\right)=x^{(i-1)}$, then the restricted hull $\mathfrak{g}:=W(1, m)_{p} \subset \operatorname{Der}_{k}(A(1, m))$ is given by $\mathfrak{g}=\bigoplus_{i=1}^{m-1} k D^{p^{i}} \oplus W(1, m)$. As noted in the introduction of [23], the algebra $\mathfrak{g}$ possesses Cartan subalgebras $\mathfrak{h}_{1}, \mathfrak{h}_{2}$ such that $\mathfrak{h}_{1}$ is toral of dimension $m$, and $\mathfrak{h}_{2}$ has dimension $p^{m-1}+(m-1)$ with $\mu\left(\mathfrak{h}_{2}\right)=1$. (One can, for instance, take $\mathfrak{h}_{2}:=\bigoplus_{i=1}^{m-1} k D^{p^{i}} \oplus \bigoplus_{j=0}^{p^{m-1}-1} k x^{(p j+1)} D$.) For $m \geq 2$ the Cartan subalgebra $\mathfrak{h}_{2}$ is not abelian. From (3.5) and (3.6) we obtain $\operatorname{rk}(\mathfrak{g})=m=\mu(\mathfrak{g})$. On the other hand, we have $D^{p^{m}}=0 \neq D^{p^{m-1}}$, so that 8.6 (2)) yields $m-\mu_{\min }(\mathfrak{g}) \geq m$. Thus, $\mu_{\min }(\mathfrak{g})=0$, and $\mathfrak{C a r}(\mathfrak{g})$ affords a $p$-nilpotent deformation. By the same token, we see that the bound given in (8.6) (2)), although often being far from optimal, can in general not be improved. (The latter fact can of course also be seen by considering nil-cyclic Lie algebras.)

Theorem 8.7. Let $(\mathfrak{g},[p])$ be a finite-dimensional restricted Lie algebra. Then the following statements hold:

(1) $\mu_{\min }(\mathfrak{g}) \geq \mu(C(\mathfrak{g}))$.

(2) If $\mathfrak{g}=\operatorname{Lie}(\mathcal{G})$ is the Lie algebra of a smooth group $\mathcal{G}$, then we have $\mu_{\min }(\mathfrak{g})=$ $\mu(C(\mathfrak{g}))$.

Proof. (1) We put $\Upsilon:=\mathfrak{C} \mathfrak{a r}(\mathfrak{g})$ and $\Omega:=\bar{\Upsilon}$ for ease of notation. The desired inequality will follow from the identity

$$
\bigcap_{\mathfrak{h} \in \Upsilon} \mathfrak{h}=\bigcap_{\mathfrak{h} \in \Omega} \mathfrak{h} .
$$


One inclusion being trivial, we consider the subspace $V:=\bigcap_{\mathfrak{h} \in \Upsilon} \mathfrak{h}$ of $\mathfrak{g}$. Owing to (7.3) the subset

$$
\mathcal{A}:=\left\{\mathfrak{h} \in \Omega ; \operatorname{dim}_{k} V \cap \mathfrak{h}=\operatorname{dim}_{k} V\right\}
$$

is closed. Since $\mathcal{A}$ contains $\Upsilon$, we have $\mathcal{A}=\Omega$, whence $V \subset \mathfrak{h}$ for every $\mathfrak{h} \in \Omega$.

As every Cartan subalgebra $\mathfrak{h} \subset \mathfrak{g}$ contains the center $C(\mathfrak{g})$, it follows that $T(\mathfrak{g}) \subset \mathfrak{h}$ for every $\mathfrak{h} \in \Omega$. Consequently, $\mu_{\min }(\mathfrak{g}) \geq \operatorname{dim}_{k} T(\mathfrak{g})=\mu(C(\mathfrak{g}))$.

(2) Now suppose that $\mathfrak{g}=\operatorname{Lie}(\mathcal{G})$ is the Lie algebra of a smooth algebraic group $\mathcal{G}$. Without loss of generality, the group $\mathcal{G}$ may also be assumed to be connected. Let $\mathcal{B} \subset \mathcal{G}$ be a Borel subgroup. The group $\mathcal{G}(k)$ acts on the projective variety $\Omega$ via the adjoint representation. By Borel's Fixed Point Theorem [4 (10.4)] there exists an element $\mathfrak{h}_{0} \in \Omega$ such that $b \cdot \mathfrak{h}_{0}=\mathfrak{h}_{0}$ for every $b \in \mathcal{B}(k)$. Accordingly, $\mathfrak{h}_{0}$ is a $\mathcal{B}(k)$ submodule of $\mathfrak{g}$. Since $\mathcal{B}(k)$ acts on $\mathfrak{h}_{0}$ via automorphisms of restricted Lie algebras, the toral radical $T\left(\mathfrak{h}_{0}\right)$ is also $\mathcal{B}(k)$-invariant. As tori are rigid (cf. 4, (8.10)]), we see that the connected group $\mathcal{B}(k)$ acts trivially on $T\left(\mathfrak{h}_{0}\right)$. Differentiation yields $\left[T\left(\mathfrak{h}_{0}\right), \operatorname{Lie}(\mathcal{B})\right]=(0)$, so that $T\left(\mathfrak{h}_{0}\right)$ is contained in the center $C(\operatorname{Lie}(\mathcal{B}))$ of $\operatorname{Lie}(\mathcal{B})$ (cf. [14, (13.3)]).

Let $\mathcal{U} \subset \mathcal{G}$ be the unipotent radical of $\mathcal{G}$. Evidently, $\mathcal{U} \subset \mathcal{B}$ (cf. [4, (11.21)]), and we put $\mathcal{G}^{\prime}:=\mathcal{G} / \mathcal{U}$ as well as $\mathcal{B}^{\prime}:=\mathcal{B} / \mathcal{U}$. Thanks to [4, (11.20)] $\mathcal{B}^{\prime}$ is a Borel subgroup of the reductive group $\mathcal{G}^{\prime}$. We consider $\mathfrak{g}^{\prime}:=\operatorname{Lie}\left(\mathcal{G}^{\prime}\right)$ and note that $\mathfrak{g}^{\prime}$ is isomorphic to $\mathfrak{g} / \operatorname{Lie}(\mathcal{U})$. It follows that the image of $T\left(\mathfrak{h}_{0}\right)$ in $\mathfrak{g}^{\prime}$ is contained in the center of the Borel subalgebra $\operatorname{Lie}\left(\mathcal{B}^{\prime}\right)$. Basic properties of the root systems of reductive groups (cf. $4, \S 14])$ now imply that $C\left(\operatorname{Lie}\left(\mathcal{B}^{\prime}\right)\right)$ coincides with $C\left(\mathfrak{g}^{\prime}\right)$. Observing $\operatorname{Lie}(\mathcal{U}) \subset \operatorname{Lie}(\mathcal{B})$, we therefore obtain

$$
(\operatorname{ad} t)^{2}(\mathfrak{g}) \subset(\operatorname{ad} t)(\operatorname{Lie}(\mathcal{U}))=(0)
$$

for every $t \in T\left(\mathfrak{h}_{0}\right)$. Since the elements of $T\left(\mathfrak{h}_{0}\right)$ are semisimple, we conclude $T\left(\mathfrak{h}_{0}\right) \subset C(\mathfrak{g})$. Consequently, $T\left(\mathfrak{h}_{0}\right) \subset T(\mathfrak{g})$, so that $\mu_{\min }(\mathfrak{g}) \leq \operatorname{dim}_{k} T(\mathfrak{g})=$ $\mu(C(\mathfrak{g}))$.

Suppose that $\mu_{\min }(\mathfrak{g})=\mu(C(\mathfrak{g}))$ and let $\mathfrak{h}_{0} \in \overline{\mathfrak{C a} \mathfrak{a}(\mathfrak{g})}$ be a $p$-subalgebra with $\mu\left(\mathfrak{h}_{0}\right)=\mu(C(\mathfrak{g}))$. As $\mathfrak{h}_{0}$ is nilpotent and contains $T(\mathfrak{g})$, we have $T\left(\mathfrak{h}_{0}\right)=T(\mathfrak{g})$. Consequently, $\mathfrak{h}_{0}$ operates nilpotently on $\mathfrak{g}$ in that case. The author is not aware of an example of a Lie algebra $\mathfrak{g}$, for which the Cartan subalgebras of dimension $\operatorname{rk}(\mathfrak{g})$ do not afford deformations acting on $\mathfrak{g}$ via nilpotent transformations.

The arguments of (8.7) also show that the number

$$
\nu_{\min }(\mathfrak{g}):=\min \left\{\mu(\mathfrak{h}) ; \mathfrak{h} \in \overline{\operatorname{Tor}_{\mu(\mathfrak{g})}(\mathfrak{g})(k)}\right\}
$$

coincides with $\mu(C(\mathfrak{g}))$ in the classical situation.

\section{Regular Points}

Let $(\mathfrak{g},[p])$ be a finite-dimensional restricted Lie algebra. We have seen in the foregoing section that the quasi-projective variety $\mathfrak{C a r}(\mathfrak{g})$ of Cartan subalgebras of minimal dimension is irreducible. The main result of this section, Theorem 9.3, identifies an open subset of the smooth locus of $\mathfrak{C a r}(\mathfrak{g})$. For solvable and simple Lie algebras of classical or Cartan type this information suffices to ensure the smoothness of $\mathfrak{C a r}(\mathfrak{g})$.

Recall that, given a torus $\mathfrak{t} \subset \mathfrak{g}$ of dimension $\mu(\mathfrak{g})$, the morphism

$$
\zeta_{\mathfrak{t}}: \mathcal{T}_{\mathfrak{g}} \longrightarrow \operatorname{Sub}_{\mathrm{rk}(\mathfrak{g})}(\mathfrak{g}) \quad ; \quad x \mapsto \tilde{\mathfrak{g}}_{0}(x)
$$


factors through to a morphism

$$
\eta_{\mathfrak{t}}: \hat{\mathcal{T}}_{\mathfrak{g}} \longrightarrow \operatorname{Sub}_{\mathrm{rk}(\mathfrak{g})}(\mathfrak{g})
$$

such that $\left(\eta_{\mathfrak{t}}\right)_{k}$ induces an injective, dominant morphism $\hat{\mathcal{T}}_{\mathfrak{g}}(k) \longrightarrow \mathfrak{C a r}(\mathfrak{g})$. We propose to show that this morphism is étale, thereby establishing that those Cartan subalgebras of $\mathfrak{C a r}(\mathfrak{g})$ having a maximal torus of dimension $\mu(\mathfrak{g})$ belong to the nonsingular locus of $\mathfrak{C a r}(\mathfrak{g})$. Thus, the centralizers of tori of maximal dimension are regular points of the variety $\mathfrak{C} \mathfrak{a} \mathfrak{r}(\mathfrak{g})$, providing additional justification for Premet's terminology [23].

Lemma 9.1. Let $\mathfrak{t} \subset \mathfrak{g}$ be a torus of dimension $\mu(\mathfrak{g})$. Then $\mathfrak{t}$ induces a morphism

$$
\zeta_{\mathfrak{t}}: \mathcal{T}_{\mathfrak{g}} \longrightarrow \operatorname{Car}_{\mathrm{rk}(\mathfrak{g})}(\mathfrak{g}) \quad ; \quad x \mapsto \tilde{\mathfrak{g}}_{0}(x)
$$

of schemes.

Proof. In view of [11, (1.4)] $\mathcal{T}_{\mathfrak{g}}$ is a smooth affine algebraic scheme, whose function algebra will be denoted $A$. Thus, $\mathcal{T}_{\mathfrak{g}} \cong \operatorname{Spec}_{k}(A)$, and $\zeta_{\mathfrak{t}}$ is the morphism associated to $\tilde{\mathfrak{g}}_{0} \in \operatorname{Gr}_{\mathrm{rk}(\mathfrak{g})}(\mathfrak{g})(A)$ via Yoneda's Lemma. It remains to show that $\tilde{\mathfrak{g}}_{0}(x) \in$ $\operatorname{Car}_{\mathrm{rk}(\mathfrak{g})}(\mathfrak{g})$ for every $x \in \operatorname{Spec}_{k}(A)(R)$ and $R \in M_{k}$.

We put $\ell:=\operatorname{rk}(\mathfrak{g})+1$, and begin by showing that $\tilde{\mathfrak{g}}_{0}^{(\ell)}=(0)$. Let $\left\{v_{1}, \ldots, v_{n}\right\} \subset \mathfrak{g}$ be a basis of $\mathfrak{g}$, and consider an element $m:=\sum_{i=1}^{n} v_{i} \otimes a_{i} \in \tilde{\mathfrak{g}}_{0}^{(\ell)} \subset \mathfrak{g} \otimes_{k} A$. Thanks to $(3.5(1))$ the subalgebra $\tilde{\mathfrak{g}}_{0}(x) \subset \mathfrak{g}$ is a Cartan subalgebra of dimension $\mathrm{rk}(\mathfrak{g})$ for every $x \in \operatorname{Spec}_{k}(A)(k)$. Consequently,

$$
\sum_{i=1}^{n} x\left(a_{i}\right) v_{i}=\left(\operatorname{id}_{\mathfrak{g}} \otimes x\right)(m) \in \tilde{\mathfrak{g}}_{0}(x)^{(\ell)}=(0),
$$

for every $x \in \operatorname{Spec}_{k}(A)(k)$, so that the ideal $\sum_{i=1}^{n} A a_{i}$ lies in the intersection $\bigcap_{x \in \operatorname{Spec}_{k}(A)(k)} \operatorname{ker} x$. In view of Hilbert's Nullstellensatz the latter ideal is the nilpotent radical of the finitely generated $k$-algebra $A$. As $\mathcal{T}_{\mathfrak{g}}$ is smooth, the algebra $A$ is reduced, implying $m=0$.

Since $\tilde{\mathfrak{g}}_{0}^{(\ell)}=(0)$, it readily follows that $\left(\tilde{\mathfrak{g}}_{0} \otimes_{A} R\right)^{(\ell)}=(0)$ for every homomorphism $A \longrightarrow R$ of commutative $k$-algebras. As a result, $\zeta_{\mathfrak{t}}$ sends $\mathcal{T}_{\mathfrak{g}}$ to $\operatorname{Car}_{\mathrm{rk}(\mathfrak{g})}(\mathfrak{g})$.

Let $\mathfrak{t} \subset \mathfrak{g}$ be a torus of dimension $\mu(\mathfrak{g})$. Since the morphism $\zeta_{\mathfrak{t}}$ is constant on the $G_{\mathfrak{t}}$-orbits of the scheme $\mathcal{T}_{\mathfrak{g}}$, it factors through to a morphism $\eta_{\mathfrak{t}}: \hat{\mathcal{T}}_{\mathfrak{g}} \longrightarrow \operatorname{Car}_{\mathrm{rk}(\mathfrak{g})}(\mathfrak{g})$. By the same token, a torus $\mathfrak{t} \subset \mathfrak{g}$ of dimension $d$ gives rise to morphisms

$$
\gamma_{\mathfrak{t}}: \mathcal{T}_{\mathfrak{g}} \longrightarrow \operatorname{Tor}_{d}(\mathfrak{g}) ; \varphi \mapsto \varphi(\mathfrak{t})
$$

and $\omega_{\mathfrak{t}}: \hat{\mathcal{T}}_{\mathfrak{g}} \longrightarrow \operatorname{Tor}_{d}(\mathfrak{g})$.

Our next result amounts to a refinement of [11, (1.4)]:

Lemma 9.2. Let $\mathfrak{t} \subset \mathfrak{g}$ be a torus of dimension $d$.

(1) The morphism $\gamma_{\mathfrak{t}}: \mathcal{T}_{\mathfrak{g}} \longrightarrow \operatorname{Tor}_{d}(\mathfrak{g})$ is étale.

(2) If $d=\mu(\mathfrak{g})$, then the morphism $\zeta_{\mathfrak{t}}: \mathcal{T}_{\mathfrak{g}} \longrightarrow \operatorname{Car}_{\mathrm{rk}(\mathfrak{g})}(\mathfrak{g})$ is étale.

Proof. We only verify (2); the proof of (1) is analogous. Let $C$ be a finitedimensional commutative $k$-algebra, $I \triangleleft C$ an ideal with $I^{2}=(0)$. The canonical projection $\pi: C \longrightarrow C / I$ induces an exact sequence

$$
(0) \longrightarrow \mathfrak{g} \otimes_{k} I \longrightarrow \mathfrak{g} \otimes_{k} C \longrightarrow \mathfrak{g} \otimes_{k} C / I \longrightarrow(0)
$$


of finite-dimensional restricted $k$-Lie algebras. To verify the lifting property, we let $\varphi \in \mathcal{T}_{\mathfrak{g}}(C / I)$ and $\mathfrak{m} \in \operatorname{Car}_{\mathrm{rk}(\mathfrak{g})}(\mathfrak{g})(C)$ be given such that $\left(\operatorname{id}_{\mathfrak{g}} \otimes \pi\right)(\mathfrak{m})=$ $C_{\mathfrak{g} \otimes_{k} C / I}(\operatorname{im} \varphi)$. Since $\mathcal{T}_{\mathfrak{g}}$ is a smooth scheme (cf. [11, (1.4)]), there exists an element $\psi \in \mathcal{T}_{\mathfrak{g}}(C)$ such that

$$
\left(\mathrm{id}_{\mathfrak{g}} \otimes \pi\right) \circ \psi=\varphi \circ\left(\mathrm{id}_{\mathfrak{t}} \otimes \pi\right) .
$$

By definition, $\mathfrak{m}$ is a finite-dimensional nilpotent restricted Lie algebra over $k$, whose unique maximal torus will be denoted $\mathfrak{t}_{\mathfrak{m}}$. Owing to [29] (II.4.5)] $\left(\mathrm{id}_{\mathfrak{g}} \otimes \pi\right)\left(\mathfrak{t}_{\mathfrak{m}}\right)$ is the unique maximal torus of the nilpotent Lie algebra $C_{\mathfrak{g} \otimes_{k} C / I}(\operatorname{im} \varphi)$. Consequently, we have $\left[\left(\operatorname{id}_{\mathfrak{g}} \otimes \pi\right) \circ \psi\right](\mathfrak{t} \otimes 1) \subset\left(\operatorname{id}_{\mathfrak{g}} \otimes \pi\right)\left(\mathfrak{t}_{\mathfrak{m}}\right)$, so that

$$
\psi(\mathfrak{t} \otimes 1) \subset \mathfrak{t}_{\mathfrak{m}}+\mathfrak{g} \otimes_{k} I=\mathfrak{t}_{\mathfrak{m}} \oplus\left(\mathfrak{g} \otimes_{k} I\right),
$$

with the latter identity following from the fact that the $p$-ideal $\mathfrak{n}:=\mathfrak{g} \otimes_{k} I$ of $\mathfrak{g} \otimes_{k} C$ is strongly abelian. Thanks to (5.1) there exists an element $x \in \mathfrak{n}$ such that

$$
[\exp (x) \circ \psi](\mathfrak{t} \otimes 1) \subset \mathfrak{t}_{\mathfrak{m}}
$$

We consider the map $\psi^{\prime}:=\exp (x) \circ \psi$. As $\exp (x)$ is an automorphism of the restricted $C$-Lie algebra $\mathfrak{g} \otimes_{k} C$ satisfying $\left(\mathrm{id}_{\mathfrak{g}} \otimes \pi\right) \circ \exp (x)=\mathrm{id}_{\mathfrak{g}} \otimes \pi$, we have

(a) $\psi^{\prime} \in \mathcal{T}_{\mathfrak{g}}(C)$, and

(b) $\left(\mathrm{id}_{\mathfrak{g}} \otimes \pi\right) \circ \psi^{\prime}=\varphi \circ\left(\mathrm{id}_{\mathfrak{t}} \otimes \pi\right)$, and

(c) $\psi^{\prime}\left(\mathfrak{t} \otimes_{k} C\right) \subset C \psi^{\prime}\left(\mathfrak{t} \otimes_{k} 1\right) \subset C \mathfrak{t}_{\mathfrak{m}} \subset \mathfrak{m}$.

The nilpotent Lie algebra $\mathfrak{m}$ is contained in $C_{\mathfrak{g} \otimes_{k} C}\left(\psi^{\prime}(\mathfrak{t} \otimes 1)\right)=\zeta_{\mathfrak{t}}\left(\psi^{\prime}\right)$. Moreover, $\mathfrak{m}$ and $\zeta_{\mathfrak{t}}\left(\psi^{\prime}\right)$ are $C$-direct (hence projective) summands of $\mathfrak{g} \otimes_{k} C$ such that $\mathfrak{m} \otimes_{C} C / I=$ $C_{\mathfrak{g} \otimes_{k} C}\left(\psi^{\prime}(\mathfrak{t} \otimes 1)\right) \otimes_{C} C / I$. Consequently, $\mathfrak{m}=\zeta_{\mathfrak{t}}\left(\psi^{\prime}\right)$, while (b) implies $\psi_{C / I}^{\prime}=\varphi$. As a result, the morphism $\zeta_{\mathrm{t}}$ is smooth.

Let $\varphi, \varphi^{\prime} \in \mathcal{T}_{\mathfrak{g}}(k)$ be rational points belonging to the same fibre of $\left(\zeta_{\mathfrak{t}}\right)_{k}$. Then $\varphi^{\prime}(\mathfrak{t}) \subset C_{\mathfrak{g}}(\varphi(\mathfrak{t}))$, so that $\varphi^{\prime}(\mathfrak{t})=\varphi(\mathfrak{t})$. Accordingly, $\varphi^{\prime}$ belongs to the $G_{\mathfrak{t}}(k)$-orbit of $\varphi$. Hence the fibres of $\left(\zeta_{\mathfrak{t}}\right)_{k}$ are finite, and we may apply [6] (I, $\left.\S 4,4.7\right)$ ] to see that $\zeta_{t}$ is étale.

Theorem 9.3. Let $(\mathfrak{g},[p])$ be a finite-dimensional restricted Lie algebra. Then the following statements hold:

(1) If $\mathfrak{t} \subset \mathfrak{g}$ is a torus of dimension $d$, then there is an isomorphism $\hat{\mathcal{T}}_{\mathfrak{g}}(k) \cong$ $\operatorname{Tor}_{d}(\mathfrak{g})(k)$. In particular, $\operatorname{Tor}_{d}(\mathfrak{g})(k)$ is a smooth, affine variety.

(2) The map

$$
C_{\mathfrak{g}}: \operatorname{Tor}_{\mu(\mathfrak{g})}(\mathfrak{g})(k) \longrightarrow \mathfrak{C a r}(\mathfrak{g}) ; \quad \mathfrak{t} \mapsto C_{\mathfrak{g}}(\mathfrak{t})
$$

is an étale morphism.

(3) Let $\mathfrak{h} \in \mathfrak{C a r}(\mathfrak{g})$ be a Cartan subalgebra. If $\mu(\mathfrak{h})=\mu(\mathfrak{g})$, then $\mathfrak{h}$ is a regular point of $\mathfrak{C a r}(\mathfrak{g})$.

Proof. (1) A consecutive application of (9.2) and (1.4) shows that the morphism $\left(\omega_{\mathfrak{t}}\right)_{k}: \hat{\mathcal{T}}_{\mathfrak{g}}(k) \longrightarrow \operatorname{Tor}_{d}(\mathfrak{g})(k)$ is étale. Since it is also bijective, it is an isomorphism (cf. [18, (3.6.3.4)]).

(2) Let $\mathfrak{t} \subset \mathfrak{g}$ be a torus of dimension $\mu(\mathfrak{g})$. Arguing as in (1) we see that $\left(\eta_{\mathfrak{t}}\right)_{k}: \hat{\mathcal{T}}_{\mathfrak{g}}(k) \longrightarrow \mathfrak{C} \mathfrak{a r}(\mathfrak{g})$ is étale. Since $C_{\mathfrak{g}} \circ\left(\zeta_{\mathfrak{t}}\right)_{k}=\left(\eta_{\mathfrak{t}}\right)_{k}$, the assertion follows directly from (1).

(3) Since $C_{\mathfrak{g}}$ is étale, it induces bijections $T_{\mathfrak{t}}\left(\operatorname{Tor}_{\mu(\mathfrak{g})}(\mathfrak{g})(k)\right) \cong T_{C_{\mathfrak{g}}(\mathfrak{t})}(\mathfrak{C a r}(\mathfrak{g}))$ (cf. [6. (I, §4, 3.4)]) between the tangent spaces at $\mathfrak{t}$ and $C_{\mathfrak{g}}(\mathfrak{t})$, respectively. As both varieties have the same dimension, $C_{\mathfrak{g}}$ takes regular points to regular points. 
Accordingly, $\operatorname{im} C_{\mathfrak{g}}=\{\mathfrak{h} \in \mathfrak{C} \mathfrak{a r}(\mathfrak{g}) ; \mu(\mathfrak{h})=\mu(\mathfrak{g})\}$ is contained in the smooth locus of $\mathfrak{C a r}(\mathfrak{g})$.

Corollary 9.4. Let $(\mathfrak{g},[p])$ be a finite-dimensional restricted Lie algebra. If all maximal tori of $\mathfrak{g}$ have the same dimension, then the variety $\mathfrak{C a r}(\mathfrak{g})$ of Cartan subalgebras of $\mathfrak{g}$ is smooth.

Proof. By assumption, all maximal tori have dimension $\mu(\mathfrak{g})$. Applying (3.1) and (3.5)(2)) we see that all Cartan subalgebras of $\mathfrak{g}$ have $\operatorname{dimension} \operatorname{rk}(\mathfrak{g})$, so that $\mathfrak{C a r}(\mathfrak{g})$ is the variety of all Cartan subalgebras of $\mathfrak{g}$. In view of $(9.3(3)) \mathfrak{C a r}(\mathfrak{g})$ is smooth.

Corollary 9.5. Let $\mathfrak{g}$ be a finite-dimensional restricted Lie algebra.

(1) If $\mathfrak{g}$ is solvable, then $\mathfrak{C a r}(\mathfrak{g})$ is smooth.

(2) If $p \geq 5$ and $\mathfrak{g}$ is simple of classical or Cartan type, then $\mathfrak{C a r}(\mathfrak{g})$ is smooth. In either case, $\mathfrak{C a r}(\mathfrak{g})$ coincides with the set of all Cartan subalgebras of $\mathfrak{g}$.

Proof. Our results follow from (9.4) once we have established that every maximal torus $\mathfrak{t} \subset \mathfrak{g}$ has dimension $\mu(\mathfrak{g})$. According to [30, (2.17)] this is the case whenever $\mathfrak{g}$ is solvable.

Now let $\mathfrak{g}$ be simple. If $\mathfrak{g}$ is classical, then [14, (5.4)] shows that either $\mathfrak{g}=\operatorname{Lie}(\mathcal{G})$ is the Lie algebra of a smooth algebraic group $\mathcal{G}$, or $\mathfrak{g} \cong \mathfrak{s l}(p n) / C(\mathfrak{s l}(p n))$ for some $n \in \mathbb{N}$ (see also [25. p. 132]). Since $C(\mathfrak{s l}(p n))=T(\mathfrak{s l}(n))$ the desired property follows from a consecutive application of (4.4) and [29] (II.4.5)].

Alternatively, $\mathfrak{g}$ belongs to one of the four series of Lie algebras of Cartan type. In this case Demuškin's results [7, 8] ensure the applicability of (9.4).

\section{ACKNOWLEDGEMENTS}

This paper was written during the author's stay as a Mercator Professor at the University of Bielefeld. He would like to take this opportunity to thank the Department of Mathematics for its hospitality, and Detlef Voigt for several stimulating and fruitful discussions.

The author would like to thank the referee for carefully reading the manuscript and providing numerous comments leading to expository improvements.

\section{REFERENCES}

[1] D. Barnes. On Cartan subalgebras of Lie algebras. Math. Z. 101 (1967), 350-355 MR 36:3837

[2] G. Benkart. Cartan subalgebras in Lie algebras of Cartan type. Canadian Mathematical Society Conference Proceedings 5 (1986), 157-187 MR 87f:17005

[3] R. Block and R. Wilson. Classification of the restricted simple Lie algebras. J. Algebra 114 (1988), 115-259 MR 89e:17014

[4] A. Borel. Linear Algebraic Groups. Graduate Texts in Mathematics 126. Springer-Verlag, 1991 MR 92d:20001

[5] D. Collingwood and W. McGovern. Nilpotent Orbits in Semisimple Lie Algebras. Van Nostrand Reinhold, 1993 MR 94j:17001

[6] M. Demazure and P. Gabriel. Groupes Algébriques I. Masson, North-Holland, 1970 MR 46:1800

[7] S. Demuškin. Cartan subalgebras of the simple Lie-p-algebras $W_{n}$ and $S_{n}$. Siberian Math. J. 11 (1970), 233-245 MR 41:6919

[8] Cartan subalgebras of simple non-classical Lie algebras. Math. USSR Izv. 6 (1976), 905-924

[9] D. Eisenbud. Commutative Algebra. Graduate Texts in Mathematics 150. Springer-Verlag, 1995 MR 97a:13001 
[10] R. Farnsteiner and D. Voigt. On cocommutative Hopf algebras of finite representation type. Adv. in Math. 155 (2000), 1-22 MR 2001e:16070

[11] Schemes of tori and the structure of tame restricted Lie algebras. J. London Math. Soc. 63 (2001), 553-570 MR 2002a:17011

[12] - On infinitesimal groups of tame representation type. Math. Z. 244 (2003), 479-513

[13] G. Hochschild. Cohomology of restricted Lie algebras. Amer. J. Math. 76 (1954), 555-580 MR 16:109a

[14] J. Humphreys. Algebraic Groups and Modular Lie Algebras. Mem. Amer. Math. Soc. 71 (1967) MR 36:169

[15] Linear Algebraic Groups. Graduate Texts in Mathematics 21. Springer-Verlag, 1975 MR 53:633

[16] J. Jantzen. Representations of Algebraic Groups. Pure and Applied Mathematics 131. Academic Press, 1987 MR 89c:20001

[17] N. Jacobson. Basic Algebra I. Freeman \& Co., San Francisco, 1974 MR 50:9457

[18] H. Kurke, G. Pfister and M. Roczen. Henselsche Ringe und algebraische Geometrie. Mathematische Monographien 11. VEB Deutscher Verlag der Wissenschaften, 1975 MR 58:10899

[19] J. Lepotier. Lectures on Vector Bundles. Cambridge Studies in Adavanced Mathematics, 54. Cambridge University Press, 1999 MR 98a:14019

[20] H. Matsumura. Commutative Ring Theory. Cambridge Studies in Advanced Mathematics 8. Cambridge University Press, 1997

[21] D. Mumford. The Red Book of Varieties and Schemes. Lecture Notes in Mathematics 1358. Springer-Verlag, 1999 MR 2001b:14001

[22] A. Premet. On Cartan subalgebras of Lie-p-algebras. Math. USSR Izvestiya 29 (1987), 145157 MR 88d:17012

[23] Regular Cartan subalgebras and nilpotent elements in restricted Lie algebras. Math. USSR Sbornik 66 (1990), 555-570 MR 90g:17017

[24] D. Quillen. Projective modules over polynomial rings. Invent. Math. 36 (1976), 167-171 MR 55:337

[25] G. Seligman. Modular Lie algebras. Ergebnisse der Mathematik und ihrer Grenzgebiete 40 Springer-Verlag, New York 1967 MR 39:6933

[26] I. Shafarevich. Basic Algebraic Geometry 1. Springer-Verlag, 1994

[27] _ Basic Algebraic Geometry 2. Springer-Verlag, 1997

[28] T. Springer. Linear Algebraic Groups. Birkhäuser Verlag, 1981 MR 84i:20002

[29] H. Strade and R. Farnsteiner. Modular Lie Algebras and their Representations. Pure and Applied Mathematics 116. Marcel Dekker, 1988 MR 89h:17021

[30] D. Winter. On the toral structure of Lie-p-algebras. Acta Math. 123 (1969), 69-81 MR 40:4326

Fakultät für Mathematik, Universität Bielefeld, Postfach 1001 31, 33501 Bielefeld, GERMANY

E-mail address: rolf@mathematik.uni-bielefeld.de 